Evaluating the MyPlate Teaching Tool in an Obese Rural Population at Risk for Type 2 Diabetes

Karen R. Dawn

Vienna, Virginia

BSN, University of Virginia, 1981

MSN, University of Virginia, 2009

A Capstone presented to the graduate faculty at the University of Virginia in Candidacy for the Degree of Doctor of Nursing Practice

\author{
School of Nursing \\ University of Virginia
}

May, 2014

Doris Glick, PhD

Signature of Chair

Audrey Snyder, PhD

Signature of Member

Ishan Williams, $\mathrm{PhD}$

Signature of Member 


\section{ACKNOWLEDEMENTS}

Nursing, like a team sport, requires a group of people to work together towards a common goal. I have an amazing Capstone team. I would like to express my deepest appreciation to my committee chair, Professor Doris Glick who provided continuous support and guidance on this project. Her insights and encouragement enabled me to achieve this goal. My deepest gratitude goes to Professor Audrey Snyder who introduced me to the rich culture and wonderful people living in Virginia's Appalachian region. She encouraged me to lead patient education during the RAM events and this role enabled me to try a new healthy eating intervention. Her extensive knowledge of the population and their health needs led to improving client education, with a focus on disease prevention and healthy lifestyle interventions. Without her support, this project would not have been possible. Professor Ishan Williams' insights and expertise with rural populations with type 2 diabetes and survey design helped guide this project towards positive results.

This project was possible due to the outstanding support and dedication of the University of Virginia RAM team. Dawn Niles, was instrumental in her willingness to retrieve omitted study participant information. The nurse educators, especially Anne Diamond, Kimberly Morris, Kim Maloney, Cindy Westley, and Lorna Roberts demonstrated creative interventions, positive role modeling and student mentorship during the RAM events. The graduate and undergraduate University of Virginia nursing students, worked tirelessly with the educators to provide client education to this vulnerable population.

Quiping (Pearl) Zhou, RN, PhD offered suburb statistical analysis, analytical support, and guidance. The George Washington University School of Nursing faculty 
and staff gave me continuous encouragement and support towards the completion of this project. A grant from The University of Virginia School Of Nursing Alumni Association was used to defray the participant incentives cost.

No team is complete with an outstanding cheering section. My family, especially my daughters Sarah and Caroline Mihm, served as the best cheerleaders throughout this process. I am eternally grateful and proud. 


\begin{abstract}
The rural population of Appalachia is plagued by enormous health disparities due to extensive poverty, lack of education, and minimal access to health care providers. Type 2 diabetes and obesity (a pre-cursor to type 2 diabetes) have become regional epidemics. There is accumulating evidence that diabetes prevention is cost-effective and that diabetes prevention interventions are beneficial from both the health system and a societal perspective. Because there are limited health care providers and many barriers to accessing treatment within the Appalachian population, the Remote Area Medical (RAM) clinic has been an important initiative in addressing the regional health needs. The purpose of this project was to evaluate the effectiveness of a teaching intervention, the MyPlate Healthy Eating Guide, used at the 2012 RAM events in Wise and Grundy, Virginia. In 2012, five nurse educators partnered with student nurses to implement a nutrition education project using the MyPlate educational tools targeting clients at risk for developing or who have type 2 diabetes. This tool offered simple, easy to understand, graphic information that significantly reduced nutrition instruction time and increased client comprehension, evaluated by response from 87 clients who completed a survey immediately following instruction. A follow-up survey was mailed to 70 clients who participated in the initial survey and agreed to be contacted for follow-up to evaluate the effectiveness of the tool on self-reported behavior change and body weight. Results demonstrate that the one-time education session using the MyPlate tool lead to weight loss in this vulnerable population, albeit not statistically significant. Barriers to following the MyPlate included food cost and change in eating habits. Participants who saw their MyPlate tool each day trended towards more weight loss than those who did not see their
\end{abstract}


tool daily. Women were more likely to lose weight than men. Providing this population with education that includes healthy, affordable, and culturally sensitive nutrition choices can lead to greater awareness about healthy nutrition and its impact on body weight and diabetes. Health care providers, nursing students, and lay health workers, who work with at-risk populations may benefit from using this simple, clear, nutrition intervention.

Key Words: obesity, nutrition, Appalachia, diabetes 
Table of Contents

Acknowledgements............................................ 2

Abstract...................................................... 4

Section I- Introduction.......................................... 8

Purpose............................................... 19

Theoretical Framework.................................... 20

Research Question....................................... 22

Section II- Review of the Literature .............................. 23

Implications for Nursing................................ 39

Rationale............................................... 40

Section III- Methods............................................ 42

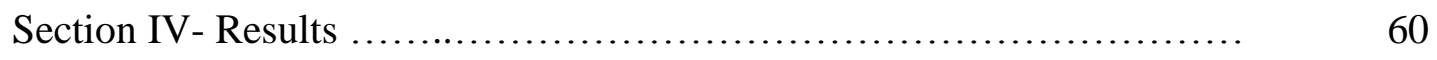

Section V- Discussion........................................... 68

References................................................ 86

Appendices.................................................. 102

Appendix A- Virginians with Diabetes who are Overweight.............. 102

Appendix B- County Economic Status in Appalachia, FY 2014.......... 103

Appendix C- Prevalence of Diabetes in Virginia Appalachia $\quad . . . . . . . . . . . \quad 104$

Appendix D- Inactive Virginia Adults ............................ 105

Appendix E- Obese Virginia Adults............................... 106

Appendix F- Health Promotion Model (revised)...................... 107

Appendix G- Table 1:AMDR................................. 108

Appendix H- MyPlate Tool....................................... 109 
Appendix I- Table 2: RAM Clients, 2012........................ 110

Appendix J- Figure 1, MyPlate Comprehension....................... 111

Appendix K- MyPlate Baseline Survey............................ 112

Appendix L- MyPlate Follow-up Survey........................... 114

Appendix M-Cover Letter...................................... 116

Appendix N- IRB Approvals..................................... 118

Appendix O- Table 3: Baseline and follow-up characteristics............ 122

Appendix P- Figure 2: Ability to Follow MyPlate, baseline and follow-up $\quad 124$

Appendix Q- Figure 3: Perceived and Actual Ability to Follow by Meal... 125

Appendix R- Figure 4: Responder Weight Loss at Baseline and Follow-up 126

Appendix S- Figure 5: Weight Change in Responders by Category........ 127

Appendix T-Table 4: Gender Difference and Weight Change............ 128

Appendix U- Figure 6: MyPlate Tool Visibility and Weight Change...... 129

Appendix V- Figure 7: Tool Visibility and Weight Change by Category.... 130

Appendix W - Author Guideline................................... 131

Appendix X - Manuscript - AJN........................... 139 


\section{Section I - Introduction}

Obesity rates in the United States (U.S.) have increased dramatically over the last 30 years, and obesity is now an epidemic in the U.S. Data for 2003-2004 and 2005-2006 indicated that approximately two thirds of U.S. adults and one fifth of U.S. children were either obese (defined for adults as having a body mass index $[\mathrm{BMI}] \geq 30.0$ ) or overweight (defined for adults as BMI of 25.0--29.9 and for children as at or above the 95\%

percentile of the sex-specific BMI for age-growth charts) (Ogden, 2006, 2010). Updated in 2012, the 2009-2010 results show a $16.9 \%$ obesity rate for children and adolescents age two through 19, which the authors considered unchanged from the previous results (Ogden, Carroll, Kit, Flagal, 2012). Seventy-two percent of men and 64 percent of women are overweight or obese, with about one-third of adults being obese (Flegal, Carroll, Ogden, Curtin, 2010). Poor diet and inactivity are the major contributors to the obesity epidemic (United States Department of Agriculture \& HHS, Dietary Guidelines for Americans, 2010).

Many chronic diseases, including type 2 diabetes, cardiovascular disease, osteoarthritis and psychological illnesses are related to obesity. Additionally, early death and poor quality of life are associated with obesity (Dixon, 2012). The Trust for America's Health (TFAH) and the Robert Wood Johnson Foundation's $F$ as in Fat Report (2013) estimates if American's diet and exercise habits are not improved, the adult obesity rate in the U.S. will rise to 50-60\% (or higher) in most states by 2030 . In addition to poor health outcomes, obesity accounts for billions of U.S dollars each year. A study done by Finkelstein, Trogdon, Cohen \& Dietzthe (2009) notes the annual obesity medical burden has risen to almost 10 percent of all medical spending and amounted to 
$\$ 147$ billion per year in 2008 and the medical costs for people who are obese were $\$ 1,429$ higher than those of normal weight individuals. As a person's BMI increases, so do the number of sick days, medical claims and health care costs associated with that person (Heithoff, Cuffel, Kennedy, Peters, 1997).

The relationship between type 2 diabetes and increase body weight and obesity has been well documented (Centers for Disease Control (CDC), 2011; American Diabetes Association, 2012; Dixon, 2012; Trust for America's Health and the Robert Wood Johnson Foundation, 2013). Diabetes affects 25.8 million people in the United States, 8.3\% of the population (CDC, 2011). According to the Virginia Department of Health (2011), there are 531,000 adults in Virginia (8.7\%) with diabetes that was diagnosed by a health professional. Appendix A shows the percent of adults in Virginia with diabetes who were overweight or obese between 1995 and 2010. Type 2 diabetes mellitus $(\mathrm{t} 2 \mathrm{DM})$ is defined as elevated blood glucose caused by ineffective endogenous insulin usage and $90 \%$ to $95 \%$ of all diagnosed cases of diabetes are this type (CDC, 2011). A precursor to t2DM is pre-diabetes, which affects 79 million Americans (35\% of U.S. adults and $50 \%$ of those over 65 years). According to the Centers for Disease Control and Prevention (2012) pre-diabetes, also known as impaired fasting glucose (IFG), is fasting glucose levels between 100 to $125 \mathrm{mg} / \mathrm{dL}$ and only $7 \%$ of those with pre-diabetes are aware they have it. Studies have shown that people with pre-diabetes who lose weight and increase their physical activity can prevent or delay type 2 diabetes (The DPP group, 2002). The Diabetes Prevention Program (DPP) was a National Institute of Health (NIH) funded study that demonstrated that the introduction of a healthy low-fat diet and physical activity of moderate intensity for at least 150 minutes per week, in conjunction 
with a weight loss goal of $7 \%$, resulted in a $58 \%$ reduction in progression to diabetes when compared with the usual-care group. This was true across all ethnic groups (white, African American, Hispanic, Asian, and American Indian) and both genders. Lifestyle changes worked especially well for participants aged 60 and older, reducing their risk of developing t2DM by $71 \%$ (The DPP group, 2002).

Poor eating habits, along with sedentary lifestyles have been documented as leading to weight gain. The National Health and Nutrition Examination Survey (NHANES) III indicated that of 1,480 individuals with diabetes, $62 \%$ ate fewer than the recommended five servings of fruit and vegetables per day and $68 \%$ consumed more than $30 \%$ of their calories from fat (Nelson, Reiber, \& Boyko, 2002).

The economic cost of diabetes is staggering and affects vulnerable populations at higher rates. According to The American Diabetes Association's (ADA) Economic Costs of Diabetes in the U.S. 2007 report, the national cost of diabetes in the U.S. exceeds $\$ 174$ billion. This includes $\$ 116$ billion in excess medical expenditures and $\$ 58$ billion in reduced national productivity (2008). A diabetes prevention project, in addition to personal burden reduction, is also cost effective. There is accumulating evidence that diabetes prevention is cost-effective and the diabetes prevention interventions are beneficial from both the health system and a societal perspective (Herman, Hoerger, Brandle, Hicks, Sorensen, Zhang, et al, 2005). In a clinical trial, comparison with placebo, costs per quality-adjusted life-year were approximately $\$ 1,100$ for lifestyle interventions and $\$ 31,300$ for treatment with metformin. From a societal perspective, the interventions cost approximately $\$ 8,800$ and $\$ 29,900$ per quality adjusted life-year, respectively. 
According to the Virginia Diabetes Council (VDC), which authored the Virginia Diabetes Plan 2008-2012 to reduce the incidence, prevalence and burden of diabetes within the Commonwealth, primary prevention of diabetes is a top priority. The prevention strategies are highlighted in initiative three: improve public competency to reduce personal risk factors for t2DM by increasing awareness about prediabetes, risk factors for $\mathrm{t} 2 \mathrm{DM}$ and the consequences of diabetes. The group highlights three goals to address the initiative. The first goal is the creation of a work group to encourage Virginians to engage in healthy lifestyles and increase screening for diabetes. The second goal is to ensure that prevention resources are accessible to all Virginians and stakeholders, and the third goal focuses on employers to implement healthy worksite lifestyle practices (Virginia Diabetes Plan 2008-2010, 2008). These goals and intervention strategies are evaluated with quarterly meetings held at varying locations throughout the Commonwealth.

\section{Appalachia Health}

The Appalachian region is a 205,000 -square-mile area that follows the Appalachian Mountains from southern New York to northern Mississippi. This region includes 420 counties, all of West Virginia and parts of 12 states: Alabama, Georgia, Kentucky, Maryland, Mississippi, New York, North Carolina, Ohio, Pennsylvania, South Carolina, Tennessee, and Virginia. Forty-two percent of the Region's population is rural, compared with 20 percent of the national population (Appalachian Regional Commission, 2012).

Appalachians are 1.4 times as likely as non-Appalachians to have diabetes, with rates highest among individuals with low incomes (Stivers, 2002). According to 
Virginia's Diabetes Control Program, from 1996 to 1999, the southwest region of the state was second highest in proportion of the number of hospitalizations for diabetes in Virginia. Historically, the people of Appalachia did not exhibit the mobility that characterized much of the rest of the United States and often remained on their ancestral land. As a result, they became isolated from the mainstream and culturally distinct from the rest of the nation (Birdsall, 2008). This isolation has impacted their view of health and utilization of health care services. Today, Appalachia has high rates of poverty, low rates of education, high rates of unemployment, an aging population, limited access to health care, high rates of cigarette smoking, and generally poor health status (Barker, Crespo, Gerzoff, Denham, Shrewsberry \& Cornelius-Averhart, 2010) (Appendix B). Virginians living in the Appalachian region have a higher prevalence of diabetes than those living in non-Appalachian areas. In 2009, diabetes prevalence was $13.9 \%$ among Appalachians, which is significantly higher $(\mathrm{p}<0.001)$ than prevalence of $7.4 \%$ among non-Appalachian Virginians (Virginia Department of Health (2011) (Appendix C). In addition to a high prevalence of diabetes, the risk for death among people with diabetes is about twice that of people of similar age but without the disease (CDC, 2011). A 2010 study estimating the effects of disease prevention on life expectancy in the Appalachia region noted that men would gain 5.7 years and women would gain 4.5 years of life if risk factor exposures (BMI, blood glucose, blood pressure and smoking) were at optimal levels (Danaei, Rimm, Oza, Kulkarni, Murray, Ezzati). For BMI improvement alone, adults living in Appalachia would gain 1.5 (male) and 1.4 (female) additional life years. An elevated BMI has an impact on cardiovascular wellness, development of type 2 
diabetes and other chronic diseases. A reduction in BMI not only reduces the development of deadly diseases, but also extends life (Danaei, et al, 2010).

Healthy People 2020 defines a health disparity as a particular type of health difference that is closely linked with social or economic disadvantage. Health disparities adversely affect populations who have experienced greater social or economic obstacles to health based on a number of factors including socioeconomic status, gender, race, or ethnic group (2008). The people of Appalachia, specifically those living in southwest, Virginia, have health disparity due to their geographic isolation and low socioeconomic status.

Barker, et al, (2010) found that Appalachians living in economically distressed counties, are at higher risk of diabetes than are residents in other Appalachian counties. In addition, residents of distressed counties in Appalachia had 33\% higher odds of reporting diabetes than residents in non-Appalachian counties. According to the Center for Disease Controls (CDC) Behavioral Risk Factor Surveillance System (BRFSS), in 2009, 32.5\% of the adult population in Wise County, Virginia were classified as inactive, a precursor to t2DM (Appendix D) and 31.6\% of the population of Wise County were obese (Appendix E), also a precursor to t2DM.

Serono, Leiferman, and Dauber (2007), qualitatively studied Appalachian residents' risk of developing diabetes among individuals who were not previously told they were at risk of developing the disease. In addition to diabetes risk, self-efficacy beliefs and selected dietary and physical activity practices that may help prevent diabetes were evaluated. The study concluded that over $63 \%$ of the sample had three or more risk factors for diabetes and that $87 \%$ reported being overweight, compared to a national 
average of $64 \%$. Also, $67 \%$ reported engaging in little or no exercise, compared to a national average of $40 \%$ and those individuals classified as high-risk were even less likely to report being physically active (Appendix D). One of three recommendations by Serono, et al, is to develop culturally appropriate, community-based diabetes prevention programs focusing on improving dietary and physical activity behaviors and self-efficacy aimed toward preventing diabetes, for at-risk individuals in medically underserved areas (2007).

According to Behringer (2006), Appalachians traditionally try to manage their own problems without outside intervention. Denham, Meyer, Toborg, and Mande, studied health education in Appalachia by interviewing people in 54 focus groups, which included 469 people, to determine ways Appalachian women engaged family members in health-promotion activities. They discovered that family was one of the most important themes within this group and both adult and youth participants indicated that women take an active role in maintaining health among the individual family members. Other cultural considerations to disseminate health information included personal contact with those sharing the health information, politely framed messages, and reliance on facts (2004).

A qualitative study (Coyne, Demian-Popescu \& Friend, 2006) examining social and cultural factors that influence health in a section of Appalachia revealed that there exists many positive attributes related to the people who live in this region. These attributes include: friendly, God fearing, proud, law abiding, hard working, and reluctant to share family problems. This unwillingness to share family problems has an impact on seeking health care, as some view the health care visit as an intrusion in their private/family lives. This intrusion is viewed as distrust in the health care provider. This 
region ties health belief to religious beliefs, although they acknowledge the relationship between health problems and health behaviors, they also view poor health as a normal part of their lives (Coyne, et al, 2006).

High rates of disease incidence and mortality in poor Appalachian regions have been linked to economic factors, but there is also a significant relationship between the cultural and social factors of the population and the health disparities that they face (Brown, et al, 2004). There is a way of life associated with many generations of families living in the region that perpetuates some unhealthy practices. Most people in Appalachia are strongly linked to place and family, with multiple generations living, working, and dying in the same area ("Diabetes in Appalachia", 2011). Extended family, kin, and long-time friends still play important roles in the daily lives of many that reside in the region. Thus, cultural values and beliefs get passed down and may contribute to increased risk for poor health among subsequent generations. For example, cultural food patterns within families motivate eating practices, and healthy dietary standards are often in conflict with typical Appalachian eating patterns - which include eating foods high in fat and refined carbohydrates. Ingrained patterns of tobacco use and physical inactivity are also significant health risks faced in the region (VDH, 2011). Furthermore, even though specialized health care services have started to develop within the main cities of the region, both the cultural and financial implications of a family relocating means that large geographic distance often prevents access to much-needed care. Undoubtedly, culture plays an important role in understanding, addressing and meeting the health care needs of the Appalachian people (VDH, 2011). 
Despite recent advances within the infrastructure of the Appalachian region and continued volunteer efforts directed toward lessening the disparities faced by the population, the region still lags behind the rest of the United States on economic and social indicators (Appalachian Regional Commission, 2012) (Appendix B). Long-term poverty and its associated stressors continue to threaten the health of the people in the area. The combination of low socioeconomic status, tobacco use, high obesity rates, limited diet choices, and physical inactivity has contributed to a culture in which poorly controlled chronic diseases are commonplace. Cultural traits tied to Appalachian traditions need to be considered in the development and use of health education materials (Denham, Meyer \& Toborg, 2004).

\section{Remote Area Medical (RAM)}

For the past 13 years, Remote Area Medical Corps (RAM), a nonprofit, international relief organization based in Knoxville, Tennessee, has sponsored a three day health care event in Wise, Virginia, in southwest, Virginia, nestled in the Appalachian south central region (Appendix B). A second RAM event, in Grundy, Virginia, which is also in the Appalachian south central region, is a one-and-a-half-day event in October each year.

Both RAM events attract people from a wide area, primarily Virginia, North Carolina, Tennessee and Kentucky. The event's primary organizer is the non-profit nurse managed, mobile health care provider in southwest Virginia, The Health Wagon. The RAM clinic held yearly in Wise, Virginia, plays a vital role for insured, low wage-earners and the uninsured, by offering services and screening exams they would otherwise forgo due to costly co-payments or lack of coverage by their insurance plan. In July, 2012, the 
Wise RAM event included 2,442 clients seen with 5,046 health care encounters including medical, mammography, eye care, and dentistry. Without these services provided by the 1,379 volunteers, many individuals within the Appalachian region would not receive any health care throughout the year (RAM USA, 2012). A study examining the 2008 Wise RAM population noted the high rates of obesity, heart and vascular disease, diabetes and arthritis that affect this region. During this event, the average BMI $(n=1,730)$ was 30.9 , which is classified as obese. Seventy-seven percent of the clients had a BMI above the normal range: $27 \%$ were classified as overweight and $50 \%$ obese (Snyder \& Milbrath, 2013). The authors recommend future health care services that target these health concerns during this annual three-day health clinic (Snyder \& Milbrath, 2013).

During each RAM event, all attendees have baseline vital signs, height, weight and random blood sugar measurements. If their blood sugar is in the diabetes range (fasting blood glucose $>125$ or random $>200$ ), then an A1C (a blood test that evaluates diabetes control over the previous three months) is also performed (American Diabetes Association, 2012).

There is a strong correlation between obesity and t2DM and many Appalachian residents have never received any healthy diet education and their excess body weight can lead to high rates of t2DM. This population's nutrition choices play a role in causing these high levels of obesity as many Appalachians fry much of their food, drink large amounts of sugar-containing soda, and do not regularly practice portion control (Denham, Manoogian \& Schuster, 2007). The top 10 foods identified as important to Appalachians are bacon, biscuits and gravy, chicken and dumplings, cornbread, coffee, fried potatoes, green beans, soup beans, stack cakes and vegetable soup (Sohn, 2005). Healthy food cost 
is a factor that inhibits Appalachians from choosing healthy foods. A recent study identified cost as a barrier to following a healthy meal plan, the Mediterranean diet, which is a plant-based, high fiber diet that reduces the risk of cardiovascular disease (Hardin-Fanning, 2012).

The prevention and management of diabetes is a complex process that involves healthy meal planning and food portion control, daily exercise, and medication. As demonstrated in the Diabetes Prevention Protocol (2002), clients at high-risk for developing t2DM or who have pre-diabetes, healthy food choices which lead to a 7\% weight loss can dramatically decrease the risk of developing t2DM. For those clients who have previously been diagnosed with t2DM, nutrition education can minimize the potential complications associated with the disease (Norris, Lau, Smith, Schmid, Engelgau, 2002).

Therefore, providing client education that is culturally sensitive, and includes healthy and affordable nutrition choices could lead to greater client awareness about healthy eating. Education for this client population should be tailored to the unique Appalachian culture and include their health beliefs and practices. By offering education about lifestyle modification that is targeted toward the Appalachian population, chronic disease prevention and diabetes management can be better geared towards this underserved region.

A Healthy People 2020 goal (D-16.3) related to diabetes prevention is to "increase the proportion of persons at high-risk for diabetes with prediabetes who report reducing the amount of fat or calories in their diet." Based on the most recent Healthy People data, extrapolated from the National Health and Nutrition Examination Survey 
(NHANES) 2000 report and adjusted for the 2005-2008 age adjusted population,

“48.5 percent of adults aged 18 years and older who were at high-risk for diabetes with prediabetes reported reducing the amount of fat or calories in their diet in 2005-08." The Healthy People target-setting method is a $10 \%$ increase to 53.4 percent who reduce fat and calories (Healthy People, 2012).

Effective diabetes treatment requires knowledge in self-management skills that include healthy eating, daily exercise, daily monitoring of self blood glucose and daily, often self adjusted, medication. The treatment plan requires the person with diabetes to be diligent in their desire to improve their health and prevent the complications that uncontrolled diabetes can cause. This diligence is improved with client education and support (Jarvis, Skinner, Carey, Davies, 2010). Diabetes education can be facilitated by the use of diabetes educators, who are trained in the intricacies of the disease process and the treatment options available to the person with diabetes. Diabetes educators are experts at fostering positive behavior change in people with diabetes, and the interventions they use are effective (Balamurugan, Ohsfeldt Hughes \& Phillips, 2006).

\section{Purpose}

The purpose of this project is to evaluate the effectiveness of an educational intervention, the MyPlate Healthy Eating Guide, used at the 2012 RAM events in Wise and Grundy, Virginia, on healthy eating behavior and body weight. At both events, clients at risk of developing t2DM, or with either pre-diabetes or t2DM, were instructed using an individually tailored MyPlate educational tool to promote healthy eating. The advantage to this type of education is several fold: 1) it is based on evidence-based findings, 2) the entire educational handouts are readily available on the internet and 
include on-line data tracking for the users, (www.myplate.gov) 3) all intervention tools are free, 4) the teaching concepts related to MyPlate are straight forward compared to other nutrition instruction methods used for diabetes education- like carbohydrate counting and the exchange diet, and 5) clients who completed a survey immediately after the RAM MyPlate teaching instruction said the tool was easy to comprehend and made healthy eating instruction comprehensible.

\section{Theoretical Framework}

There are a limited number of diabetes education programs that have documented using a theoretical framework as a basis for the educational program. Whittemore (2006) lists the following cognitive and behavioral theories that can be applied to diabetes selfmanagement: behavior modification theory, cognitive behavior theory, skills empowerment theory, Health Belief Model, Health Decision Model, motivational interviewing, self-regulation theory, readiness to change, social cognitive theory, stage of change theory, stress and coping theory, theory of planned behavior, and theory of reasoned action. The Health Promotion Model by Pender (Appendix F) was used as the theoretical framework for this evaluation study. The Health Belief Model is loosely associated with the Health Promotion Model (HPM). A Cumulative Index to Nursing and Allied Health Literature (CINAHL) search with the terms "Health Promotion Model" and “diabetes” from 2003-present yielded nine results.

The HPM focuses on three areas: individual characteristics and experiences, behavior-specific cognition and affect, and behavioral outcomes (Pender, Murdaugh, Parsons, 1996). The model is a guide to study what motivates an individual to engage in behaviors that enhance health. The HPM is a competence or approach-oriented model 
that does not include fear as a means of motivation. In the revised HPM, there are three new variables: activity-related affect, commitment to a plan of action, and immediate competing demands and preferences (Appendix F) (Pender, Murdaugh, Parsons, 2011). For this project, the commitment to a plan of action, which relates to a commitment to carry out a specific action at a given time and place (in this case, eating) and identifying strategies for carrying out the new behavior. Pender notes "commitment alone without associated strategies often results in good intentions but failure to perform the health behavior" (Pender, et al, 2011, p. 49).

An important aspect of the HPM is tailoring behavior change interventions to the individual. According to Rimer \& Kreuter (2006) tailoring creates individualized communication to meet the needs of an individual. Health materials should be individualized and based on an initial assessment. Tailored materials are more effective than generic materials and are more likely to be read and viewed at a later date (Kreuter, Caburnay, Chen \& Donlin, 2004). Using the HPM as a guide for a targeted nursing client needs assessment and designing individualized teaching materials and interventions will help the client make healthy changes that have the potential to improve overall health.

Studies based on the HPM indicate that perceived barriers (79\%), interpersonal influences (57\%), and situational influences (56\%) were some of the important determinants of health-promoting behavior (Pender, Murdaugh, Parsons, 2006). This implies that nurses need to understand and address these variables when empowering persons with diabetes (Ho, Berggren, Dahlborg-Lyckhage, 2010).

Motivational interviewing is a means of working with lifestyle changes in health promotion practice. The basic plan of motivational interviewing is to help people 
understand their lifestyle problems and make positive lifestyle changes (Miller \& Rollnick, 2011). According to a study by Brobeck, Bergh, Odencrants and Hildinghusing (2011) motivational interviewing was used to guide health promotion practice interventions among primary care nurses. This study determined that the nurses' experience with motivational interviewing was an enriching and useful method that promotes awareness and guidance in the care relationship and it proved to be more effective than conventional methods in increasing client motivation.

The Health Promotion Model's focus on improved wellness through positive behavior change, and the use of motivational interviewing, which has also proven effective in eliciting healthy behaviors, are used to guide the individualized and targeted nursing assessment and teaching MyPlate intervention for the clients at the RAM events. These models were chosen because they address and accept the current health level of each client, while guiding the client towards healthy choices. The people of Appalachia, as previously documented, practice many poor health choices and both the Health Promotion Model and motivational interviewing focus on positive changes that can be worked into the client's routine versus focusing on the negative behavior exhibited by the client.

\section{Research Question}

The purpose of this project is to evaluate the effectiveness of a one-time educational intervention, the MyPlate Healthy Eating Tool, used at the 2012 RAM events in Wise and Grundy, Virginia. Did vulnerable, obese clients at risk for type 2 diabetes living in Appalachia who had a one-time teaching session using the MyPlate Healthy Eating Tool have improved eating behaviors that led to weight loss? 


\section{Section II - Literature Review}

\section{Statement of the Problem}

The Appalachian region of southwest Virginia is experiencing epidemic rates of obesity and t2DM (Behavioral Risk Factor Surveillance System, 2010: Baker, et al, 2010). Many individuals in this region are uninsured and their health care is delivered by a volunteer mobile clinic. Can a one-time MyPlate nutrition education tool taught at the 2012 RAM clinic improve eating behaviors and body weight?

\section{Literature Summary}

\section{Diabetes Education and its impact on health outcomes}

Ovid Medline was used to identify review articles related to type 2 diabetes education, health outcomes and compliance. Search terms included: "client education" and "type 2 diabetes" from 2005 to present and were narrowed to include research and English language articles only. Forty-three articles were identified. Ten were eliminated due to either relating to a specific diabetes medication (eight studies) opinion/commentary (one) or a web-based education program (one). Three Cochrane Database of Systematic Reviews (Deakin, McShane, Cade, Williams (2009); Duke, Colagiuri, Colagiuri (2009); Hawthorne, Robles, Cannings-John, Edwards (2008)) are included. Two comprehensive reviews and meta-analysis: Loveman (2008), Wens (2008) are also included. Additional studies were identified through reference lists, specifically targeting dietary or behavior change interventions, which are beneficial to this particular project.

Whittemore (2006) acknowledges there are many interventions to support behavioral change in diabetes self-management. Interventions that are multifaceted, 
tailored to the individual and provide support for six months or longer, demonstrate improvement in diabetes-related outcomes. Improving access to diabetes selfmanagement education and addressing the more complex social and environmental factors that contribute to behavior change are current challenges in diabetes selfmanagement. According to Deakin, McShane, Cade and Williams (2009), nutritional intake and lifestyle modifications are the cornerstone for treating t2DM.

The American Diabetes Association updated the guidelines for diabetes selfmanagement education (DSME). DSME is defined as the ongoing process of incorporating knowledge, skill, and ability necessary for diabetes self-care (Funnell, Brown, Childs, Haas, Hosey \& Jensen, 2012). This process incorporates the needs, goals, and life experiences of the person with diabetes and is guided by evidence-based standards. The "overall objectives of DSME are to support informed decision-making, self-care behaviors, problem-solving and active collaboration with the health care team and to improve clinical outcomes, health status, and quality of life" (Funnell, Brown, Childs, Haas, Hosey \& Jensen, 2012, p. S101). The new standards (ten) relate to structure, process and outcomes. The task force concluded, based on clinical evidence, that programs which incorporate behavioral and psychosocial strategies demonstrate improved diabetes outcomes. Additional studies show that culturally and age-appropriate programs improve diabetes outcomes. In addition to content, personnel for the DSME were also reviewed. Effective DSME included registered nurses, registered dieticians and pharmacists as the primary educators. All were effective in delivering diabetes education. Assessment of the individual with diabetes is a crucial aspect of DSME, as the education is individualized to meet the needs of the person with diabetes. 
A systematic review by Sumlin and Garcia (2012) summarized research looking at culturally competent food related interventions for African American women with type 2 diabetes. Out of the fifteen studies reviewed, most of the interventions addressed food habits as one of many components for diabetes control. The authors speculated that the research participants may have been overwhelmed with the breath and variety of information presented to them. The authors recommend targeted interventions that focus on dietary changes as the foundation for diabetes self-management education (DSME) for African American women.

A meta-analysis on the effect of self-care management intervention in t2DM compiled by Menet, Moller, Vach, Wagner \& Henriksen (2010), noted a plausible tendency towards shorter educational interventions to be more effective than longer interventions. The authors speculate that shorter interventions allow participants to remember and better synthesize the diabetes education information. The authors recommend that additional and larger studies be conducted with a long follow-up period to establish knowledge about the educational intervention effects over time. In addition to time, content was also reviewed. Nau (2012) reports that less complex diabetes treatment regimens are associated with higher rates of adherence to diabetes treatment programs. Anderson and Fennell (2010) discussed the importance of empowerment and diabetes care and education. Empowerment is helping clients enhance and use their own innate ability to gain mastery over their diabetes. They speculated that healthcare providers, who are trained in the treatment of acute care illnesses, are not properly trained in client empowerment. This lack of training has a significant impact on client practice. "Self-reflection occurring in a relationship characterized by psychological safety, 
warmth, collaboration, and respect is essential for laying the foundation for self-directed positive change in behavior, emotions, and/or attitudes" (Anderson \& Fennell, 2010, p. 281). Joy (2008) lists strategies for diabetes management to support and encourage client adherence to therapeutic regimens. These involve education of clients and health care providers, client follow-up procedures, a teamwork approach for clients and providers, and counseling and teaching techniques such as Motivational Interviewing.

Jarvis, Skinner, Carey and Davies (2010), note that diabetes is a complex disease that is difficult to manage without diabetes education. Most diabetes management is completed by the person with diabetes independently, outside the clinical setting, i.e., their home environment. Diabetes management is extremely complex, requiring education related to diet, exercise, medication management and self-management. Due to these complex management steps, people with diabetes may benefit from a diabetes education program. Many education programs can be adapted to ethnic minority populations, who are affected by diabetes at higher rates than non-minorities. This focused education can reduce inequalities that may improve adherence to the diabetes treatment plan.

Assessing diabetes education cost effectiveness has proven complicated due to several factors. This is due in part to the complexity of the disease, the treatment options and most importantly, the client population the educational program is to benefit. Two systemic reviews were identified that address cost effectiveness of diabetes education. Boren, Fitzner, Panhalkar, Specker (2009) performed a literature review to determine if diabetes education is cost effective. The review included 26 research articles from 19912006. Most of the 26 studies (18) determined that diabetes education was cost effective, 
offered a cost savings and was viewed as a positive healthcare cost investment. The overall findings note that the benefits of education on diabetes self-management and lifestyle modification for people with diabetes are positive and outweigh the costs associated with the education. A second review by Urbanski, Wolf and Herman (2008), focused on the cost effectiveness of diabetes education and medical nutrition therapy (MNT). The authors note that there are a limited number of controlled trials that include full cost analyses of diabetes education, but based on what was reviewed, diabetes education is cost effective. In regards to MNT, there is a small body of evidence evaluating the cost-effectiveness of this specific therapy in diabetes care.

A Cochrane Review by Duke, Colagiuri and Colagiuri (2009), compared randomized controlled and controlled clinical trials evaluating individual education for people with T2DM. Nine trials were identified that fit the selection criteria of individual face-to-face education, compared to controls who received usual care or group education. The authors note that a major limitation was the lack in number and quality of the studies available for the review. Only four of the trials compared individual education to usual care- where clients were not given individualized education. Individual education when compared to usual care did not have a significant impact on glycemic control as measured by $\mathrm{A} 1 \mathrm{C}$ at 6 to 9 months or 12 to 18 months. For the individual education, the face-toface time was limited in most studies, with an average of two to four hours over a six month period. Group education appeared to have a greater impact on glycemic control than individual education at 6 to 9 months with a weighted mean difference of $\mathrm{HbA} 1 \mathrm{C}$ of $0.8 \%$ (95\% CI 0.3 to $1.3, \mathrm{P}=0.0007)$. There was no significant difference between the interventions at 12 to 18 months. For the purpose of this paper, only two studies looked 
at self-management outcomes. Of the two studies, only one evaluated changes in food choices. In the Whittemore (2004) study, women who participated in the individual intervention demonstrated significantly better diet self-management $(\mathrm{P}=0.02)$ with a trend towards better exercise self-management $(P=0.13)$.

A Cochrane Database Review analyzed eleven randomized controlled trials on culturally appropriate health education for t2DM in ethnic minority groups. The review found short-term effects on glycemic control, measured by $\mathrm{A} 1 \mathrm{C}$, and diabetes knowledge and healthy lifestyle. A1C improved following culturally appropriate education at three and six months compared to usual care, this was not significant at 12 months. Knowledge scores improved at three, six, and 12 months. (Hawthorne, Robles, CanningsJohn, Edwards, 2008). The eleven trials were randomized controlled trails totaling 1,603 enrolled clients. Ten trials were used in the meta-analysis. The education intervention varied from one visit to up to 12 month follow-up, none were long term, with many having interventions from six to twelve weeks.

A study by Sigurdardottir, Jonsdott and Benediktsson (2007) was one of the few studies that evaluated theory based educational interventions on outcomes. No statistically significant difference in reduction in A1C level was found between theory guided interventions and non-theory guided interventions. As the interventions were based on different theories it is difficult to compare the theories and decide which ones are most effective to guide diabetes education. The authors also report that teaching methods or content did not influence reduction in A1C level. Compared to the control group, seven interventions out of 18 achieved more than $10 \%$ relative reduction in $\mathrm{A} 1 \mathrm{C}$ level, and the intervention groups' A1C level was on average 6-7\% lower than the 
control group's. For baseline A1C less than 7.9\%, an education intervention achieved a small change in $\mathrm{A} 1 \mathrm{C}$, from +0.1 to $-.07 \%$. For initial A1C greater than $8 \%$, a significant drop in $\mathrm{A} 1 \mathrm{C}$ was found, -0.8 to $-2.5 \%$. Duration, educational content and intensity of education did not predict changes in $\mathrm{A} 1 \mathrm{C}$ levels.

Scollan-Koliopoulos, O’Connell and Walker (2005) recommend a framework for addressing the multigenerational impact of t2DM. Diabetes, particularly, t2DM is directly related to family history. A review of the literature, from 1984-2004, was performed to locate legacies of chronic illness. Two theories were used to guide the development of a framework of multi-generational legacies of diabetes: Family-systemsillness-disability and Model and Theory of Illness Representation. A legacy of diabetes is defined as awareness that diabetes is hereditary. The authors believe that individuals who understand that diabetes is hereditary may have a legacy of diabetes, which might impact their own self-care behavior. Recognition of a legacy of diabetes by healthcare providers may improve client-provider interactions and offer insight into factors that influence self-management behavior.

Gaede and Pedersen (2005) encourage health care providers to treat diabetes aggressively to reduce the risk of acute and long-term complications related to t2DM. Although this was not a study, the authors noted the increased risk of long-term complications related to diabetes; including eye, kidney, cardiovascular and neuropathy diseases. These complications are all preventable with behaviors that improve glycemic control, which include a balanced meal plan, regular physical activity, medication management, and self-monitoring of blood glucose. The inclusion of these behaviors into a daily routine can be quite challenging for many people with t2DM. The authors' 
recommendations include simple but focused behavioral modeling with continuous education, motivation and trouble-shooting.

A Cochrane Review by Deakin, McShane, Cade and Williams (2009) evaluated group t2DM education programs by evaluating randomized controlled trials and controlled trials. Participants in the education programs had improved A1Cs in the short term (four to six months) and long term, 12 months or greater. There is also no evidence to suggest that the program is more effective if delivered by a physician, dietitian or nurse as long as the health professional is trained to deliver diabetes education. Programs based on therapeutic client education using the principles of empowerment, participation and adult learning proved to be effective.

\section{Diabetes Prevention Efforts}

Two important lifestyle intervention studies have supported the proposition that t2DM can be prevented. The Finnish Study, (Tuomilehto, Linstrom, Erisson, Valle, Hamalainen, Ilanne-Parikka, et al, 2001) a randomized controlled trial of 522 middleaged obese adults, who had impaired glucose tolerance (IGT), were randomized to either brief diet and exercise counseling (control) or intensive individualized instruction on weight reduction, food intake, and information on increasing exercise (intervention). After 3.2 years, there was a $58 \%$ reduction in the incidence of diabetes in the intervention group compared with the control group. A second randomized controlled trial, The Diabetes Prevention Program (DPP), which included 3,234 obese adults with IGT, 45\% were minorities: African American or Hispanic, proved almost identical results to the Finnish Study. In the DPP, subjects were randomized into one of three treatment arms: 1) intensive nutrition and exercise counseling, 2) metformin group or 3) placebo group. 
Group 2 and 3 included standard diet and exercise recommendations. After 2.8 years follow-up, a 58\% reduction in the progression to t2DM was seen in the lifestyle group, and a $31 \%$ reduction in the metformin group, compared with the control group (Knowler, 2002).

A community-based study focused on t2DM prevention: the Ackermann, Finch, Brizendine, Honghong, \& Marreroet (2008) DEPLOY study involved 92 urban residents in a randomized controlled trial testing a community program, administered through the local YMCA. The intervention included matched paired groups, by YMCA, and the intervention group attended classes with a goal of a 5-7\% weight loss and 150 minutes of exercise each week. The control group was given information on diabetes risk and a YMCA membership. Results showed a $6 \%$ weight loss in the intervention group and $2 \%$ in the control group, $\mathrm{p}<0.001$. Reductions were also seen in total cholesterol. A second community-based program, by Hill, De Zapien, Staten, McClelland, Garza, MooreMonroy, et al (2007) involved the REACH community change model to prevent diabetes. In this program, five Arizona coalition groups moved from coalition work to policy advocacy for the community. This was done through securing community buy-in by encouraging broad civic participation. The five coalitions had an impact on the environment and policy, which moved from a pragmatic focus to a policy and advocacy focus and included healthy changes from food served in public schools and vending machines to developing walking trails in communities. The long-term health effects of these interventions have not been studied.

According to the American Diabetes Association's position statement on Prevention or Delay of Type 2 Diabetes, "Diabetes prevention policies that focus on 
lifestyle modifications, specifically modest weight loss and increased physical activity, are also very likely to have additional health benefits. Public health messages....should encourage behavior changes to achieve a healthy lifestyle" (ADA, 2004, p. s32). The 2012 Standards of Medical Care for people with diabetes from the American Diabetes Association recommends implementation of the following guidelines to prevent t2DM:

- Among individuals at high-risk for developing type 2 diabetes, structured programs that emphasize lifestyle changes that include moderate weight loss $(7 \%$ body weight) and regular physical activity (150 min/week), with dietary strategies including reduced calories and reduced intake of dietary fat, can reduce the risk for developing diabetes and are therefore recommended.

- Individuals at risk for type 2 diabetes should be encouraged to achieve the U.S. Department of Agriculture (USDA) recommendation for dietary fiber (14 g fiber $/ 1,000 \mathrm{kcal}$ ) and foods containing whole grains (one-half of grain intake).

- Individuals at risk for type 2 diabetes should be encouraged to limit their intake of sugar-sweetened beverages. (ADA, 2012, p. s16)

According to a literature review on cost effectiveness of t2DM prevention, by

Urbanski, Wolf and Herman (2008), of the five published analyses of lifestyle

interventions, four found that lifestyle intervention was cost-saving or resulted in a modest expenditure per life-year or quality adjusted life-year gained. For diabetes treatment, review of four published studies of intensive glycemic management for t2DM suggests that diabetes prevention is more cost-effective than diabetes treatment.

\section{Dietary Guidelines and Medical Nutrition Therapy}

The Dietary Guidelines are jointly developed by the Department of Agriculture (USDA) and the Department of Health and Human Services (HHS) and issued every five years (U.S. Department of Agriculture and U.S. Department of Health and Human Services, 2010). The Dietary Guidelines are written based on an expert panel of scientists and academics, who reviewed 130 research-based questions, reviewed pattern modeling 
analyses, and existing evidence-based reports. Public comments were also incorporated into the guidelines. Based on the rigorous research, literature review, use of evidencebased practice and public input, it can be summarized that the 2010 Dietary Guidelines meets rigorous scientific standards for quality nutritional recommendations and is the "gold standard" for healthy nutrition intake for Americans. The Social Ecological Framework for Nutrition and Physical Activity Decisions was used by the expert panel to identify the influence of the environment on nutrition and fitness. Appendix G shows the Acceptable Macronutrient Distribution Ranges (AMDR) for Americans, including distribution of carbohydrates, protein and fat content throughout the lifespan.

The Dietary Guidelines (USDA \& HHS, 2010), were developed to encourage healthy living through balanced nutrition. With the increased incidence of overweight and obesity, the 2010 Dietary Guidelines encourage Americans to become more conscious of what and how much they eat. This means increasing awareness of what, when, why, and how much they eat, deliberately making better choices regarding what and how much is consumed, and becoming more physically active. The Dietary Guidelines recommend that individuals focus on total number of calories consumed, monitor food intake, choose smaller portions when eating out, prepare, serve, and consume smaller portions of foods and beverages, especially those high in calories, eat a nutrient dense breakfast, limit television and computer screen time, dietary patterns low in calorie density, which is characterized by a relatively high intake of vegetables, fruit, and dietary fiber and a relatively low intake of total fat, saturated fat, and added sugars. Strong evidence shows that eating patterns that are low in calorie density improve weight 
loss and weight maintenance, and also may be associated with a lower risk of type 2 diabetes (USDA \& HHS, 2010).

Medical Nutrition Therapy (MNT) for diabetes treatment has a vast amount of evidence supporting the effectiveness of this therapy in changing dietary behaviors that improve metabolic control, blood glucose, A1C, and cardiovascular risk factors (American Dietetic Association, 2008). According to the Standards of Medical Care for people with diabetes (American Diabetes Association, 2012), MNT is a vital part of the diabetes treatment plan. The following is recommended:

- Individuals who have prediabetes or diabetes should receive individualized MNT as needed to achieve treatment goals, preferably provided by a registered dietitian familiar with the components of diabetes MNT.

- Weight loss is recommended for all overweight or obese individuals who have or are at risk for diabetes.

- For weight loss, either low-carbohydrate, low-fat calorie-restricted, or Mediterranean diets may be effective in the short-term (up to 2 years).

- Physical activity and behavior modification are important components of weight loss programs and are most helpful in maintenance of weight loss. (ADA 2012, p.s23)

The position statement further describes MNT as a form of therapy that involves many members of the diabetes treatment team, especially the person with diabetes.

Wolf, Conaway, Crowther, Hazen, Nadler, Oneida, et al (2004) completed a 1year randomized controlled trial comparing usual medical care to usual care plus lifestyle case management provided by a registered dietitian (RD). People who received lifestyle case management had substantially greater weight loss, reduced hemoglobin A1C, and decreased prescription medication use, compared to usual medical care. 


\section{My Plate}

According to the Department of Agriculture (2011), MyPlate is part of a larger communications initiative based on 2010 Dietary Guidelines for Americans to help consumers make better food choices. The 2010 Dietary Guidelines for Americans is the federal government's evidence-based nutritional guidelines to promote health, reduce the risk of chronic diseases, and reduce the prevalence of overweight and obesity through improved nutrition and physical activity. The recommendations are intended to be an integrated set of advice to achieve an overall healthy eating pattern. MyPlate is designed to remind Americans to eat healthfully; it is not intended to change consumer behavior alone. MyPlate illustrates the five food groups using a familiar mealtime visual, a place setting, see Appendix H: MyPlate, U.S. Department of Agriculture, 2011.

The MyPlate campaign, features selected messages to help people focus on key behaviors. Selected messages include:

- Enjoy your food, but eat less.

- Avoid oversized portions.

- Make half your plate fruits and vegetables.

- Switch to fat-free or low-fat (1\%) milk.

- Make at least half your grains whole grains.

- Compare sodium in foods like soup, bread, and frozen meals - and choose foods with lower numbers.

- Drink water instead of sugary drinks. (US Dept of Agriculture, 2011)

\section{MyPlate Education}

There is very little literature about the effectiveness of the MyPlate teaching tool. Most of the current literature relates to details about the launch of MyPlate, its predecessor, The Food Pyramid, media coverage, social media use with MyPlate and its appeal to young adults. Four months after the launch of MyPlate by the USDA, Uruakpa, 
Moeckly, Fulford, Hollister and Kim (2013) surveyed 51 young adults to determine their knowledge of the MyPlate guidelines. Fifty percent of the participants were aware that MyPlate replaced the MyPyramid graphic for consumer education. The majority of these participants did not understand the MyPlate guidelines.

A study by Wansink \& Kranz (2013) questioned 497 mothers through an on-line survey assessing MyPlate knowledge and practice related to the MyPlate tool and its message. Mothers who found the MyPlate message easy to understand were more likely to follow the recommendations. Those who already incorporated vegetables into their diet were more likely to believe that MyPlate's recommendations would help them eat healthier. The ability to follow the MyPlate recommendations was influenced by personal food preferences, involvement of children in food preparation, overall food preparation, nutritional knowledge, and prior knowledge and familiarity of MyPyramid.

According to Post, Eder, Maniscalco, Johnson-Bailey, \& Bard (2013), MyPlate has launched social media campaigns to target new audiences. In early 2013, MyPlate information expanded its availability to include Pinterest, Facebook and Twitter (@MyPlate). Pinterest has partnered with MyPlate to encourage healthy recipe sharing with Pinterest users. MyPlate's Facebook page includes recipes and healthy eating tips with its "Foodie Friday" weekly link. The twitter link sends out two to three tweets each day related to healthy eating, physical activity tips and healthy food purchases.

A study completed in 2013 evaluated the use of a seven-week, biweekly MyPlate text messages (Mobile MyPlate) on healthy eating behaviors among college students. Students were in one of two groups: text messages (for seven weeks) or sent a brochure on healthy eating through the mail. This study evaluated the effect of receiving repetitive 
educational text messages containing the MyPlate icon and USDA's Dietary Guidelines on college students' knowledge as well as fruit and vegetable consumption behavior. A pre-and post-online survey assessed the students' knowledge of the MyPlate food groups, and fruit and vegetable consumption. The intervention resulted in greater recognition of the MyPlate food groups $(\mathrm{p}<0.05)$ and more consumption of vegetable as compared to the control group (O'Connor, Brown, \& Savaiano, 2013).

A 2012 study by Bobroff, Minton, Diehl, Diaz, Keith, Medina-Solorzano, \& Gylfadottir assessed older Latino adult responses to the MyPlate for Older Adults miniposter for Latinos, MiPlato para Adultos Mayores (MPAM). Evaluation was done through focus groups of 47 Latinos, 60 years or older. Focus group participants found MPAM to be informative, culturally appropriate and appealing. Participants made recommendations for inclusion of additional culturally relevant foods into the MyPlate graphic, including papaya and fresh fish.

\section{Diabetes Health Outcomes and Social Economic Status}

Diabetes health outcomes are related to many factors, including social economic status and access to healthcare services (Brown, 2004). Shea and colleagues found that improved access, which was measured by a greater number of primary care providers in a region or the availability of facilities that provide high quality primary care, may reduce the negative association between income inequality and self-reported health (2000). Compared with uninsured adults with diabetes, those who were insured, had three times the odds of having undergone a dilated eye examination, which is a benchmark for diabetes care and should be performed annually (Beckles, Engelgau, Narayan, 1998). Additionally, the uninsured people with diabetes receive fewer foot examinations and 
preventive health care services. Lower socioeconomic status also effects glycemic control in persons with diabetes. Gregg, Geiss and Saddine (2001) report that uninsured adults with diabetes have poorer glycemic control compared to adults with diabetes who are insured. Studies show that improving the health of diabetes clients depends on keeping them within a structured health-care system (Boddiger, 2006). A lack of medical follow-up can lead to significant deterioration including kidney damage, increasing the risk that they would need dialysis in the future.

\section{Summary of the Literature Review}

Diabetes is an epidemic in the United States, specifically in the Appalachian region which has higher rates than most areas of the country. Numerous studies have demonstrated that $\mathrm{t} 2 \mathrm{DM}$ is a preventable disease related to a seven percent weight loss and healthy behavior change. Community education programs that incorporate healthy lifestyles and that are geared toward the individual have been effective in preventing t2DM.

Evaluating the effectiveness of a diabetes program based on outcome measures was difficult to quantify for most authors in this literature review. This was due in part to a lack of standardized diabetes education related to goals, duration, content and population. Overall, the literature demonstrated that diabetes education is effective in improving diabetes control and metabolic outcomes related to improved success. With improved control comes less risk of complications related to diabetes later in life. Diabetes education has also been proven to be cost effective.

Diabetes education and the tools used to educate should be simple, tailored to the individual and relevant to the client. In regards to diabetes education, the American 
Diabetes Association recommends that registered dieticians teach MNT, but a Cochrane Review saw no evidence to suggest that one healthcare provider was more effective than another in diabetes education. To date, there are few published outcomes on the effectiveness of the MyPlate method, this may be related to its relatively recent release date - June 2, 2011.

\section{Implications for Nursing}

Nurses are the most trusted profession as measured by a yearly Gallop poll (2013). With this trust comes responsibility. Keeping clients and the public healthy is a nursing role that carries significant responsibility. The majority of the people of Appalachia are overweight and at an increased risk for developing preventable diseases, particularly t2DM. Interventions to prevent weight gain and encourage weight loss should be implemented by nurses in one on one interaction and /or at the community/public health level. Nurses benefit from having client teaching tools that are easily accessible, based on scientific evidence, cost effective, culturally appropriate, and comprehensible. More specifically, nurses who live and work in the Appalachian region have a better understanding of the cultural nuances that pertain to eating. By using the MyPlate teaching tools and incorporating the health promotion model and motivational interviewing, nurses in this region are poised to offer healthy eating instruction that is more likely to be followed by the clients, as the nurse is a trusted, reliable resource.

A recent study by Silva-Smith, Fleury \& Belyea (2013) evaluated the effects of a theory-based, 8 week, physical activity and healthy eating nursing intervention on stroke risk in overweight and obese adults. Both the control and intervention groups were given health information: the intervention group received community face to face activity and 
healthy eating support and education. The control group received mailed, written material over the 8 weeks. The dietary measures were not statistically significant between the control and intervention group, but the intervention group consumed a higher quantity of vegetables, which has clinical significance.

\section{Rationale}

In order to more effectively control the t2DM epidemic in Appalachia, more aggressive educational campaigns must be initiated in the region related to the prevention and management of the disease. In particular, individuals who are at high-risk of developing t2DM or are newly diagnosed are prime candidates for education about lifestyle modification, especially healthy nutrition choices. Studies have shown that people with pre-diabetes who lose weight and increase their physical activity can prevent or delay type 2 diabetes (The DPP group, 2002). This approach has the potential to significantly increase their quality of life. Education must appeal directly to the targeted population and take into account low health literacy and the Appalachian culture and norms. For the population of Appalachia, the RAM clinic is an ideal setting for reaching a large population in a short period of time in order to affect lifestyle changes, particularly in the area of healthy nutrition. It is also a prime locale for getting these individuals set up with appropriate and accessible follow-up care as they learn to manage their health in the long term.

People with diabetes in the Appalachian region have unique issues and barriers related to their health and lifestyle behaviors. In Appalachia, family is an important part of health, and women take an active role in maintaining health among the individual family members. Other cultural considerations related to health messages for this 
population: personal contact with those sharing the health information, politely framed messages, and reliance on facts.

Currently, there are very limited diabetes resources targeted towards Appalachian culture (ARC, 2011). Therefore, an appropriate solution must take into account the issues of low health literacy and cultural practices to address the unique educational needs within this population. One valuable tool that can be easily distributed is a visual handout of the "MyPlate method" which is a meal planning approach that promotes consistent high fiber carbohydrate, low fat meals for blood glucose control and heart health (U.S. Department of Agriculture, 2011).

\section{Research Question}

The purpose of this project is to evaluate the effectiveness of a one-time educational intervention, the MyPlate Healthy Eating Tool, used at the 2012 RAM events in Wise and Grundy, Virginia. Did vulnerable, obese clients at risk for t2DM, living in Appalachia who had a one-time teaching session with the MyPlate Healthy Eating Tool have improved eating behaviors that led to weight loss? 


\section{Section III - Methods}

\section{Introduction}

A convenience sample was used to test healthy eating knowledge and practice in a group of clients seen at the 2012 RAM mobile clinics in southwest Virginia. At that clinic, 87 participants completed a baseline survey offered immediately after the instruction, which addressed current eating practices and perceived success and barriers following the MyPlate tools' recommendations. Follow-up surveys were mailed via U.S. Postal Service one year after instruction to the 70 participants who agreed to be contacted.

\section{Research Design}

This convenience sample used a survey with a pre and post design (quasiexperimental study) without a control group. Data collection occurred at baseline, at the time of the intervention to assess participants' eating habits and ability to make healthy nutrition change. The same data were collected one year post intervention. The people in this region move frequently due to economic vulnerabilities and job changes; therefore steps were taken to improve survey return. This study only looked at one group of individuals who received the intervention. This study design allows educators to make inferences on the effect of the teaching intervention by analyzing the pre and post survey results.

\section{Purpose}

The purpose of this project is to evaluate the effectiveness of an educational intervention, the MyPlate Healthy Eating Guide, used at the 2012 RAM events in Wise and Grundy, Virginia. At both events, clients at risk of developing t2DM, or with either 
pre-diabetes or t2DM, were instructed using an individually tailored MyPlate educational tool to promote healthy eating. The advantage to this type of education is several fold: 1) it is based on evidence-based findings, 2) the entire educational handouts are readily available on the internet and include on-line data tracking for the users, (www.myplate.gov) 3) all intervention tools are free, 4) the teaching concepts related to MyPlate are straight forward compared to other nutrition instruction methods used for diabetes education- like carbohydrate counting and the exchange diet, and 5) clients who completed a survey immediately after the RAM MyPlate teaching instruction said the tool was easy to comprehend and made healthy eating instruction comprehensible.

The goal of the follow-up survey is to evaluate the effectiveness of the one-time MyPlate teaching method as a means of addressing nutrition education in vulnerable, high-risk populations, with limited access to health care.

\section{Project Question}

The question this project aims to answer is: did clients who were educated on the MyPlate Healthy Eating Tool have improved eating behaviors that led to weight loss?

\section{Setting: The RAM education environment}

For the past 13 years, Remote Area Medical Corps (RAM), a nonprofit, international relief organization based in Knoxville, Tennessee, has sponsored an annual three day health care event in Wise, Virginia, located in southwest, Virginia. This RAM event attracts people from southwestern Virginia, North Carolina, Tennessee and Kentucky. The event's primary organizer is the Health Wagon, a mobile health care provider in southwest Virginia. The RAM clinic plays a vital role for insured, low wage- 
earners and the uninsured, by offering services and screening exams they would otherwise forgo due to costly co-payments or lack of coverage by their insurance plan.

A second RAM event was held in Grundy, Virginia for one-and-a-half-days in October, 2012. At this event clients received many of the same services that are available at the Wise RAM events, including primary medical care, and pharmacy, dental and optometry services.

\section{RAM Participants / Population}

For many of the RAM clients, these events are the only opportunity to receive free health and dental care and many traveled for hours and slept in their cars overnight to attend the events (University of Virginia Health Systems, 2012). Without these services provided by volunteers, many individuals within the Appalachian region would not receive any health care throughout the year. Lack of preventative health care, risk of chronic diseases related to excessive body weight, and lack of regular health check-ups puts this population at risk for poor health outcomes related to acute and chronic illnesses. For this reason, a simple, easy to comprehend, positively-tailored intervention that focuses on chronic disease prevention and management is needed for this population. During the 2012 Wise RAM event, a total of 2,442 clients were seen for 5,046 client encounters (RAM USA, 2012). During each RAM event, all attendees are assessed for baseline vital signs, height, weight and random blood sugar measurements. If their blood sugar is in the diabetes range, then an A1C is also performed. Common health concerns identified for the adult medical clients seen at the Wise and Grundy RAM events include: diabetes, hypertension, arthritis, poor dentition, depression, heart disease, obesity and pulmonary diseases. The most recent available RAM data which tracked diabetes and 
weight found that of the 1,510 clients seen, $66 \%$ had diabetes or a family history of the disease, $22 \%$ had either diabetes or pre-diabetes, $50 \%$ were obese and $27 \%$ were overweight $(\mathrm{N}=1,730)$. This elevated $\mathrm{BMI}$ in $77 \%$ of the attendees is above the national average of 66\% (Snyder \& Milbrath, 2013).

\section{RAM Educators and Nursing Students}

The 2012 Wise RAM event had five nurse educators, all registered nurses, who are educated in client assessment and well trained in tailoring educational interventions to meet the needs of the individual clients. Four of the five educators are Certified Diabetes Educators (CDE) who possess comprehensive knowledge and experience in diabetes management, pre-diabetes, and diabetes prevention (National Certification Board for Diabetes Educators, 2012). Each educator was assigned to a medical team and was responsible for evaluating each client seen by team. In Grundy, there was one client educator, a CDE, who partnered with two graduate nursing students to educate the clients seen in the medical clinic for the event. The nursing students were educated in client assessment, motivational interviewing and teaching the MyPlate method and assessing client comprehension after the education.

The Wise event nursing students were from The University of Virginia and included undergraduate and entry level graduate students in various stages of their nursing education. Nursing students worked in partnership with the nurse educators to assess and educate the clients seen in the medical clinic. Because of the high prevalence of diabetes, diabetes education was a significant part of the treatment provided by the educators and students. Diabetes self-management education (DSME), gives people with diabetes the knowledge, skills, and tools they need to effectively manage their diabetes 
(National Diabetes Education Program, 2009). There are seven guiding principles to guide diabetes education: healthy eating, being active, self blood glucose monitoring, taking medication, problem solving, reducing risks and healthy coping (American Association of Diabetes Educators, AADE 7, 2012).

During the October, 2012 Grundy RAM event, UVA Health System clinicians treated 143 clients (University of Virginia Health Systems, 2012). Table 2 (Appendix I) summarizes the clients seen at both RAM events.

\section{Intervention}

\section{Client assessment.}

Client assessment is the key to guide the teaching intervention. Each educator/client encounter included a client assessment. The student nurses were instructed in this educational/behavioral assessment. Motivational interviewing was used to guide the open-ended discussion and to ascertain the nutritional habits (guiding principles) of the client. Diet related questions about a typical day that are asked during the assessment included:

1. "Have you seen a change in your weight during the past year?

2. Tell me how you start your day?

3. What time do you awaken?

4. What is your first meal? (time, location amount, including beverages)?

5 . When is your second meal?

6. Do you eat in-between the first and second meal?

7. What is the next time you eat and what does it consist of?

8. Who does the cooking in your house?"

Activity is also assessed with the following questions:

1. "What type of work do you do?

2. Tell me about your activity throughout the day? Include: household activities, hobbies, gardening, hours of sleep." 
Blood glucose monitoring was also assessed: type of meter, frequency of monitoring and ranges of blood glucose by time of day. Medication management, including all diabetes and non-diabetes medications was reviewed with each client, including dosage, administration times, rationale for taking the medication, side effects, purchasing location, generic versus trade name and frequency of taking the daily medications. If insulin is used, an assessment of syringe brand, needle length, syringe reuse, insulin storage, insulin brand, how long the insulin vial is used, insulin injection sites, with a physical assessment of the injection sites was performed.

Diabetes medical follow-up was assessed by asking participants who is their usual health care provider and how often are their visits? If there is no primary care provider, a list of diabetes care providers was given to the clients, with an address, phone number and website, if applicable. Prevention of diabetes related problems was assessed and included foot exams and self foot care, dental care (self and dentist) and assessment of diabetes long-term complications, including eye disease (when and where a dilated eye exam was done) and kidney involvement. Cigarette smoking was also assessed, as this is a high health risk for the population of Appalachia (Wewers, Ahijevych, Chen, Dresbach, Kihm, Kuun, 2000). Client coping and support were also assessed and included questions related to food preparation, medication regimens, daily diabetes routines related to activity, food intake, costs associated with the care and assistance with medical follow-up. These assessment questions helped the educators and students identify the clients' strengths related to healthy behavior. 


\section{Client education intervention.}

The educational needs varied for this Appalachian population, but due to the high rates of obesity and overweight people at the RAM clinics, most client education encounters focused on healthy eating. The MyPlate handouts, obtained from the MyPlate.gov website, were distributed during the 2011 and 2012 RAM events by the nurse educators, undergraduate and clinical nurse leader (CNL) and other graduate nursing students. Each of the five nurse educators used the MyPlate educational tools exclusively for diet and nutrition education. These handouts were both easy to comprehend and allowed the nurse educator to individualize them for client use. They were simple and gave a new meaning to the phrase, "a picture is worth a thousand words." In addition to the handouts a MyPlate 2 foot by 3 foot poster was displayed above the education table for easy reference and viewing. The colorful graphic easily showed the learner that $50 \%$ of their plate should contain vegetables and fruits. The instruction time varied from client to client based on many variables including age, educational background, number and extent of health problems, and food preparation abilities. On average, the MyPlate instruction lasted approximately fifteen minutes per client encounter. This intervention fits well within the Health Promotion Model, which focuses on an individualized client assessment and targeted interventions based on the learning needs of the individual.

Student nurses were an important part of the client education process. The nursing students were a combination of third and fourth year undergraduates and graduate students. Students were partnered with the nurse educators for a three-hour rotation. After several teaching observations, and an introduction to healthy eating education using 
the MyPlate tools and motivational interviewing techniques, the students were assigned clients to assess and educate. The nurse educators were available as back-up, if the student needed assistance or had questions.

After the assessment, the educators and nursing students presented the client with the MyPlate handout that had a clear visual picture of a plate with the recommended portions of vegetables, fruits, lean proteins, and grains. Further, one handout listed recommended sources of each type of food. For example, suggested non-starchy vegetables included cucumbers, carrots, cabbage, green beans, tomatoes, etcetera. Serving sizes were also provided on the handout as well as alternative seasonings to sodium (U.S. Department of Agriculture, 2011). The nurse educator and nursing students would explain the MyPlate method and then ask the client if they enjoyed any of the recommended foods and whether they had access to these types of foods. Many of the clients had large gardens where they grew the suggested foods, so these foods were strongly reinforced. The nurse educators and nursing students also worked with the clients to find small ways they could improve their food choices, such as baking rather than frying, and reducing the amount of sugar containing beverages they consumed.

The pre-printed MyPlate handouts were $8 \frac{1}{2} \times 11$ inches with the graphic occupying the top $50 \%$ of the page. The bottom $50 \%$ was used for independent goal setting based on client input and nursing assessment. To further educate with the MyPlate, 8 inch paper plates were divided into the four MyPlate colors with a colored magic marker that corresponded to the color-coded MyPlate design. Client food preferences for each of the four plate food groups were written on the plate. Clients were instructed to take the MyPlate graphic home and place it on the refrigerator for two 
reasons: 1) to generate family support and 2) to have a daily reminder of healthy eating choices and portion size. The education emphasis was on making small changes over time to create lifestyle improvement that would lead to improved diabetes control, with a long- term goal of a $7 \%$ weight reduction to decrease the likelihood of developing t2DM in those at risk.

At the end of each education session, the nurse educator and/or nursing students asked the client to make nutritional goals for themselves to gauge their understanding of the presented information. If the clients were able to state goals such as "drink less soda" or "use different seasonings other than salt," or "make half my plate fruit and vegetables," the educator could conclude that the client had at least understood small changes they could make in meal planning to improve their nutrition and future diabetes management or disease prevention.

The graphic and MyPlate handouts were well received by all learners, both men and women and people of all ages, including adolescents and elderly. Some comments from learners included, "I don't think I get enough fruits and vegetables," "Why can't my meat take up half my plate?" "This picture makes it easy to see what I should eat," "I never eat vegetables with every meal," and "I've never even tried diet soda".

\section{Process evaluation.}

Each client who was instructed in the MyPlate method was offered a brief written survey to determine if this type of nutrition education was preferable to previous nutrition education they had received and if they thought this type of healthy eating could be sustained after instruction (Appendix K). Questions \# 6 and 7 used a Likert scale and this proved challenging for many clients as they did not understand the scale and an 
explanation was required for comprehension. For example, a Likert scale question related to "How I eat" and another question asking "Will I be able to eat like this" with 1=Never and 5=Always, some clients who said they eat like this currently, will not follow this after the instruction. This inconsistency and the amount of explanation that was required of the Likert scale is the reason it is omitted from the follow-up survey. A check-off list replaced the Likert scale for both questions on the follow-up survey.

\section{Sample Population}

During the 2012 Wise RAM event, 68 adult clients, who were seen in the medical clinic, were educated on the MyPlate method, either by the nurse educators or student nurses, and completed the written survey (Appendix K). During the 2012 Grundy RAM event, 19 clients who were educated on the MyPlate method completed the MyPlate survey. All subjects were over 18 years of age, mentally competent and included both males (28) and females (59). The respondents average BMI was 31, which is defined as obese (Ogden, 2006). Results of the initial survey demonstrate that participants thought the MyPlate method was easy to understand (based on question one on the initial survey, where $99 \%$ of the respondents responded that it was easy to understand) and $74 \%$ answered that it was easier to comprehend than previous meal plan education methods. In 2012, of the 87 initial survey responders, a maximum of 70 , responded that they were willing to be contacted for a follow-up survey.

This small sample size serves as a pilot study to determine if the MyPlate educational tool is an effective method of healthy eating instruction that elicits a behavior change. 


\section{Measures}

\section{Survey tool.}

The follow-up survey tool (Appendix L) was developed by the researcher based on the initial survey given to and completed by the clients at the time of the MyPlate instruction. The Likert scales that were on the original survey were removed from the follow-up survey and response options were simplified, without changing the meaning or interpretation of the intervention. Each survey question is summarized below. Many of the questions are duplicated from initial to follow-up survey and were chosen to determine if this type of instruction is perceived as beneficial to the client and if the client has changed eating habits as a result of the My Plate teaching.

A systematic review by Edwards, Roberts, Clarke, DiGuiseppi, Pratap,Wentz \& Kwan (2002) on increasing response rates for mailed surveys was used as a guide to generate a robust survey response in this vulnerable population. According to Battaglia, et al, (2008) survey response rates have declined greatly over the years, so extra care was taken to improve response rate in this population. The following steps were taken to increase survey response: short questionnaire (nine questions), personalized letter with colored ink, survey printed on colored paper, self-addressed stamped return envelopes, first class envelope delivery, accenting university and academic involvement, financial incentive (gift card upon receipt of completed survey), and sending non-respondents a second copy of the questionnaire and cover letter.

The follow-up survey questions and the rationale for each question are as follows: 
- Question one asks if the MyPlate tool is still with the client and if so, where is it located. A study evaluating an educational tool's effectiveness determined that if the tool was posted as a visual reminder, diabetes outcomes were improved (Levetan, Dawn, Robbins, Ratner, 2002)

- Question two relates to the amount of time the MyPlate portion sizes are followed, divided into the following categories: Always, Most of the time, Half of the time, Some of the time, or Never. These categories directly correspond to the Likert scale on the original survey.

- Question three assesses the reason the client cannot follow the MyPlate, with common barriers listed: dislike food choices, cost, big change in eating habits, or lack of family support. Clients are told to check all that apply. This identification of perceived barriers can help to improve educational interventions for future use in similar rural populations. This perceived barrier assessment fits into the Health Promotion Model.

- Question four asks about following MyPlate by meal: Breakfast, Lunch, Dinner/Supper. This can help identify which meals are easier to follow the MyPlate recommendations. If a particular meal is more challenging to follow, educational interventions can be tailored to address this particular meal(s).

- Question five assesses the client's desire to eat healthier, which relates to their motivation. This is important to assess as it identifies ones readiness to change behavior.

- Questions 6 assesses current weight. 
- Question 7 assesses weight change and was included to determine if the participant's weight has changed since their MyPlate instruction. This provides a quantifiable indicator of success or failure of the My Plate approach.

- Question 8 assesses any additional diet/healthy eating instruction since RAM, 2012. Different types of diets were included for easy check-off. There is space included for a non-participation response. This question helps to identify factors external to this project that may impact eating habits.

- Question 9 was added after analysis of results from the initial survey determined that only $52 \%$ of the respondents checked any of the chronic disease options on the survey.

The baseline and follow-up paper and pen surveys were chosen for a variety of reasons and include: low cost to implement, accessibility to the client population for follow-up, responder convenience, and the possibility that the respondent will provide more reliable information in writing - which is viewed in a non-threatening or judgmental manner. Several disadvantages related to self-administered surveys include the following, with solutions in parentheses: potential low response rate (an incentive for returning completed forms was offered), participants may not respond to all questions (more sensitive questions placed at the end of the survey), must be literate to complete the survey (respondents were encouraged to seek help in filling out the survey), may not return within the time period (incentive is time dependent), self selection bias including respondents who are not following the MyPlate instruction (the cover letter addresses the confidentiality of the survey and explains that the purpose is to test the MyPlate educational tool, not the respondent). 


\section{IRB Approval}

This study was approved by the University of Virginia Institutional Review Board for Health Sciences Research, protocol \# 17004 on October 8, 2013 (Appendix N). A second approval was obtained on December 4, 2013, allowing a follow-up mailing to the initial non-responders.

\section{Procedure}

After approval from The University of Virginia Institutional Review Board (\#17004), the 70 participants who completed the initial MyPlate survey and agreed to follow-up, were contacted by U.S. Postal Service. The follow-up paper and pen survey (Appendix L), and cover letter (Appendix M), were approved by the University of Virginia IRB-HSR, and mailed to participants. The Notice of Privacy Practices form was collected and signed by all participants during their RAM medical visit and is part of their existing medical record.

\section{Protection of Human Subjects}

Client confidentiality was protected by coding the survey responses and separating them from the client record. The mailed follow-up client survey (Appendix L) was coded numerically, without any client identifier on it to protect confidentiality. For example: Jane Doe is coded \#101, John Smith is coded \#102, until all participants were given a separate number. The coded responses were correlated to the original survey response which contain only the client medical record number as the client identifier. The initial survey was coded with the numeric code for confidentiality.

The follow-up survey was returned to the researcher in a self-addressed, postagepaid envelope to one address at the University of Virginia, School of Nursing. All 
returned follow-up surveys are kept in a locked cabinet at the School of Nursing at the University of Virginia. The participants were reminded in the cover letter not to put their name on the survey (the survey is identified by blue paper). Only the de-identified survey responses were used by the researcher. Data has been coded and entered into an excel database. Only aggregate data is reported.

\section{Study Benefits and Risk}

The risk to study participants is minimal, as client identity is not included in the analysis of the survey response. A possible risk would be client anxiety related to a request to fill out a survey that they may not wish to complete. Another potential risk is possible client confusion; the participant may not remember the MyPlate intervention or instruction, and the survey could lead to confusion about the services they received at the RAM event.

The study may benefit the individual by highlighting their dietary habits over the past several months, which may impact their dietary health behaviors. The larger benefit will be generalizable knowledge that will be applicable to similar populations (rural, diabetes, pre-diabetes, and economically depressed) pertaining to health education and nutrition/lifestyle behaviors.

For the RAM event, the nurse educators are RNs and nursing students, not nutritionists. Streamlining client education to this vulnerable and chronically ill population is beneficial not only to the client, but also to the volunteer staff, as it allows for faster, more consistent, evidence-based, and individualized nutrition education. Another benefit is that the analysis can guide future teaching methods that can be incorporated into the RAM events and the Health Wagon. 


\section{Client Incentive - Compensation}

To encourage maximum participation in the survey, an incentive ( $\$ 25$ Food City gift card) was offered once the survey was returned. The goal was a 50\% return rate on a total of 70 clients who agreed to be contacted for survey follow-up.

\section{Data Management}

Each client who completed the initial paper and pen survey and agreed to be contacted with a follow-up survey was mailed the follow-up survey (on blue paper) and cover letter. The mailed follow-up client survey (Appendix L) was coded numerically prior to mailing to the participant, without the client's name or address on it to protect confidentiality. The coded surveys were correlated to the original survey which contains only the client medical record number as the client identifier. The initial survey was coded with the numeric code for confidentiality and the survey results were entered into an excel computer database. All follow-up surveys were originally returned to the University Of Virginia School Of Nursing and kept in a locked cabinet, in a locked office. All data that was entered into a computer database were de-identified.

\section{Data Analysis}

The results of the follow-up paper and pen survey answers were analyzed and compared to the initial survey responses. Behavior change was compared from baseline survey results to follow-up results. The following questions were consistent from baseline to follow-up: \#8 baseline, \#3 follow-up and related to reasons the MyPlate 
method could not be followed after instruction and \#9 baseline and \# 4 follow-up, relating to ability to follow the MyPlate by meals: breakfast, lunch and dinner. Followup survey question $\# 2$ will be used for further education program development, as it illuminates how this educational tool is used by the client in their home environment. Height, weight and calculated BMI were compared from baseline to follow-up. While the follow-up weights are "stated," this has been found to be a reliable indicator of true weight (Shapiro \& Anderson, 2003).

All data, including baseline and follow-up survey responses were entered into the excel computer program and the appropriate statistical tests were performed in SPSS (Statistical Product and Service Solutions), version 21 computerized statistical program. Descriptive statistics were calculated for all variables. Height, weight and calculated BMIs were compared for each participant at baseline and follow-up with mean and standard deviation calculated. The "Yes / No" and "True/False" questions are nominal variables and were analyzed using a non-parametric test for the dependent variable group. Responses were coded as ordinal dependent variables " 0 ," " 1 ," and " 2 ," respectively.

\section{Strengths and Weaknesses of the Design}

This quasi-experimental (also known as the pre-post intervention) design often is used to evaluate the benefits of specific interventions. The primary advantage to this study is that it evaluates an intervention used within a vulnerable population who is at high-risk for developing complications and early death related to their body weight, composition and obesity. This convenience sample illuminates the eating habits and behavior change in this population and can help health care providers and client educators in the region offer nutrition interventions targeted to the population. The 
advantages of this convenience sample are: low cost, easy to administer, and convenient for the researchers and study participants. There are several disadvantages to the convenience sample. First, the results of a convenience sample are not representative of the greater population. Second, the results may be difficult to replicate.

\section{Nursing Practice Implications}

This study design allows educators to make inferences on the effect of the teaching intervention by analyzing the pre and post survey results. Understanding the impact of the MyPlate tool on healthy eating guides nursing assessment and interventions in populations at risk for $\mathrm{t} 2 \mathrm{DM}$ and obesity. 


\section{Section IV - Results}

\section{Educator and Student Response}

At the time of the MyPlate intervention, the nurse educators were asked how this healthy eating tool compared to other nutrition teaching methods. The comments from the RAM nurse educators related to the MyPlate teaching tool:

- Less 1:1 client education time,

- Encourages individual, family and group discussions and interactions,

- Focuses on the positive behavior change versus negative behaviors,

- Interactive - especially with food models, paper plates, and tool evaluation assessment,

- Not as complicated as other methods: exchanges, pyramid, carb counting, glycemic index,

- Instruction is independent of registered dietician.

A brief survey sent via Survey Monkey to the University of Virginia nursing students who attended the 2012 RAM events, highlighted the following student findings:

- $50 \%$ had never taught 1:1 nutrition prior to the RAM events,

- $50 \%$ had never taught nutrition in a community setting,

- $100 \%$ said that compared to other nutrition teaching tools, the MyPlate tool took less client education time,

- $100 \%$ said the tool was effective for portion size instruction,

- $100 \%$ said the tool was effective for teaching Healthy Food choices. 


\section{Participant Survey Response}

A total of 70 clients who were seen and educated on the MyPlate method during both 2012 RAM events agreed to follow-up and had available contact information. A two page, single-sided blue survey and a two page, single-sided cover letter were sent to the seventy potential study participants, along with a pre-printed, self-addressed stamped envelope addressed to the study team at The University of Virginia. The initial cover letter requested a survey return date by November 14, 2013. Participants who completed and returned the survey were informed they would receive a $\$ 25$ gift card to Food City, a popular grocery store chain in southwest Virginia and neighboring states.

The first mailing generated nine completed surveys. There were two "return to senders" (UVA School of Nursing) by the November 14, 2013 request date. An IRB expedited review was requested to send a follow-up survey and cover letter to all nonresponders. An additional three surveys were returned prior to the second mailing, for a total of 12 returned surveys with the first mailing. Fifty-six surveys were sent via postal mail on December 6, 2013 to the original non-responders, with omission of the two "return to senders." A return date of December 31, 2013 was requested, with a guaranteed $\$ 25$ gift card to Food City to participants who returned the completed survey by $12 / 31 / 13$. This second mailing generated an additional 22 survey returns and four additional "return to sender." This equals a 53\% survey response rate (the total survey return was $34 / 64$ potential returns (70-6 "return to sender") $=53.1 \%$ ). One of the 34 returned surveys was eliminated from the total, as the participant stated his weight did not change; his baseline weight was recorded as 191 pounds and the follow-up weight was 
130 pounds. A follow-up letter was sent to this participant, with the $\$ 25$ gift card, asking him to verify weight, but no response was received.

\section{Baseline survey results}

Baseline survey results demonstrated that $99 \%$ of the responders found the MyPlate tool easy to comprehend. Eighty-four percent said their family would be able to follow the tool. Ninety-six percent said they could follow this tool when eating at home, while only $64 \%$ responded they could follow this when eating out. Compared to other diet methods, the MyPlate tool was easier to comprehend, $74 \%$ versus 5\% (22\% had no opinion) (figure 1, Appendix J).

Participants were asked at baseline if they would be able to follow the MyPlate. Using a Likert scale, $60 \%$ said they would be able to follow the MyPlate recommendations. No participants said they could not follow the recommendations.

\section{Descriptive Results}

The baseline and follow-up demographic data are represented in Table 3

(Appendix O). The average age of the 84 participants who answered this question on the baseline survey was 46 (range: 19 to 78 years). Sixty-seven percent $(n=58)$ of the 87 participants at baseline were females and for follow-up $73 \%(n=24)$ were females. The mean BMI at baseline was 32.34 (SD 8.25), range 17-56. The follow-up BMI mean was 31.41 (SD 7.61), range 22-57. Further BMI breakdown shows that $21 \%(n=18)$ had a BMI $<25$ (considered normal) at baseline, compared with $26 \%(n=8)$ at follow-up. At baseline, $23 \%(n=19)$ were classified as overweight $(B M I>25-29.99)$ and $19 \%(n=6)$ at follow-up. At baseline, 56\% $(\mathrm{n}=47)$ were classified as obese $(\mathrm{BMI}>=30)$, similar to follow-up of $55 \%(n=17)$ of the respondents. 
The ability of each client to follow the MyPlate portion size was assessed at baseline and follow-up. At baseline, 26\% $(n=22)$ said they follow the MyPlate portion size most or all of the time, while at follow-up $39 \%(n=12)$ said they follow portion size most or all of the time. Even though a higher percentage of responders said they followed MyPlate portion size at follow-up, $42 \%(n=13)$ said they follow MyPlate portion size some of the time or never, compared to $16 \%(n=14)$ at baseline (Figure 2, Appendix P).

Based on the follow-up results, respondents identified several barriers to following the MyPlate including cost and a big change in eating habits. Among the follow-up respondents, $46 \%(\mathrm{n}=15)$ said that cost was a barrier to following MyPlate, compared to $19 \%(n=17)$ who perceived this as a barrier at baseline. A McNemar test was used to test significance between both groups and was not found to be significant, probability $=.250$. Another barrier to following MyPlate was, "a big change in eating habits." At baseline, 10\% $(n=9)$ identified this as a barrier, whereas, 30\% $(n=10)$ identified it at follow-up, McNemar = 1.0. A perceived barrier at baseline was a dislike of MyPlate food choices, $11 \%(n=10)$, but no one reported this at follow-up. The cost of MyPlate healthy food options and change in eating habits were barriers that were stated more at follow-up than at baseline.

The ability to follow MyPlate by meal was also assessed. Participants were asked at baseline "I can follow MyPlate for breakfast," "I can follow MyPlate for lunch," and "I can follow MyPlate for dinner/supper," with yes/no responses. At follow-up, the questions were, "I follow MyPlate for breakfast," "I follow MyPlate for lunch," and "I follow MyPlate for dinner." 
Initially participants perceived that they would follow MyPlate more than they actually did. At the time of the MyPlate instruction, participants thought that following MyPlate for all meals would be manageable: $85 \%(n=74)$ for breakfast, $89 \% \quad(n=74)$ for lunch and $98 \%(n=78)$ for dinner. For follow-up, 59\% $(n=16)$ of respondents noted that MyPlate was difficult to follow for breakfast, compared to $48 \%(n=14)$ for lunch and 43\% ( $\mathrm{n}=12)$ for dinner/super (Figure 3, Appendix Q). Participants reported that breakfast was the most challenging meal to follow. This may be related to the MyPlate graphic which includes traditional lunch and dinner pictured plates versus breakfast plates.

Participants were asked at baseline and follow-up if they wanted to eat healthier. The baseline and follow-up results were similar with $96 \%(n=81)$ answering affirmatively at baseline and 93\% (n=28) at follow-up. Additional diet instruction was assessed at follow-up to determine if this had an impact on weight loss. Of the 31 responders who answered this question, four had additional diet instruction.

The presence of chronic diseases that are related to high BMIs were assessed at baseline and follow-up. Out of the 87 total individuals seen at both the Wise and Grundy RAM events who completed a survey at the time of the MyPlate education, thirty-five responded that they had diabetes. Many of the respondents did not answer the question related to medical conditions; of the 87 surveys, forty-six responded that they had a chronic condition. The percentage who reported diabetes increased from $38 \%$ at baseline to $49 \%$ at follow-up. There were five responders who reported diabetes at the time of follow-up who did not include diabetes at baseline. Of the five, four did not include any medical condition at baseline; this question may have been omitted by these individuals. People with diabetes may have been more likely to complete the follow-up survey due to 
personal knowledge about healthy eating and its relationship to blood sugar control. The percentage of respondents who had hypertension decreased slightly from $52 \%$ at baseline to $46 \%$ at follow-up. There were three responders who reported hypertension at the time of follow-up who did not report this at baseline. None of these responders claimed any chronic condition at baseline.

\section{Outcome Results}

Perceived weight loss was assessed by asking the participants if they lost weight from the time of the 2012 RAM event. Eighty-one percent of the participants said they lost weight. Stated weights were used in follow-up and these were compared to baseline weights for all responders. The stated weight was subtracted from the baseline weight to determine weight loss change. Based on the recorded weight change, $57 \%$ of the responders lost weight. The mean weight of the responders at baseline was 195.03 pounds ( $\mathrm{SD}=42.47$ ). The mean weight at follow-up was 190.67 pounds ( $\mathrm{SD}=45.62)$. The difference ( 4.36 pounds weight loss) is not statistically significant, $t=1.45, \mathrm{df}=31$, $\mathrm{p}=0.158$ (Figure 4, Appendix R). The lack of significance could be due to the small sample size. The weight change ranged from -58 to +34 pounds. It was further classified into three categories: weight loss of five or more pounds $(n=17,53.1 \%)$, stable (within five pounds) $(\mathrm{n}=6,18.8 \%)$ and weight gain (gained five or more pounds) $(\mathrm{n}=9,28.1 \%)$. These results are summarized in Figure 5 (Appendix S).

The relationship between gender and weight loss was explored using Fisher's exact text (due to the limited number of respondents). Among males, four (44.4\%) lost weight, while five (55.6\%) gained weight from baseline to follow-up. Among females, $13(56.5 \%)$ lost five or more pounds from baseline to follow-up, six (26.1\%) remained 
stable, and four $(17.4 \%)$ gained five or more pounds. It appeared that females were more likely to lose weight in comparison with males, Fisher's exact $=5.24, \mathrm{p}=0.074$. However, due to the small sample size, the result is not statistically significant. The details are summarized in Table 4 (Appendix T). The relationship between age and weight loss was also explored using the Pearson $\mathrm{r}$ correlation. There is no significant relationship between the two variables, $\mathrm{r}=0.11, \mathrm{p}=0.543$.

The location in the home of the MyPlate tool was assessed at the one-year followup. People who kept the MyPlate tool visible reported more weight loss; however, this difference is not statistically significant. Seventeen clients had their MyPlate visible and the average weight loss was 6 pounds (SD14). Fourteen clients did not see their MyPlate tool daily and their mean weight loss was 2.9 pounds (SD21.36) $\mathrm{t}=0.49, \mathrm{df}=29, \mathrm{p}=0.63$. This is detailed in Figure 6, Appendix U. In addition to weight loss and MyPlate visibility, the relationship between BMI change and MyPlate visibility was also assessed. The mean BMI change for the participants who did not have the MyPlate visible was 0.49 (SD3.87). The mean BMI change for those who had the MyPlate visible was -0.876 $(\mathrm{SD} 2.038) \mathrm{t}=0.49, \mathrm{df}=29, \mathrm{p}=0.63$.

Further analysis was done on the relationship between MyPlate visibility and weight loss by categories: lost five or more pounds, stable within five pounds, or gained five or more pounds. Sixty-five percent (65\%) of the participants who had their MyPlate tool visible on a daily basis lost five or more pounds, compared to $43 \%$ of the group who did not see their MyPlate tool each day. Chi-square was used and results are summarized in Figure 7, Appendix V, chi-square $=7.27, p=0.104$, Cramers V=0.337, $p=0.122$. 
Although the results were not statistically significant, the Cramers' V indicates a moderate effect size. 


\section{Section V - Discussion}

The Appalachian region is plagued with high rates of poverty, obesity and preventable chronic diseases, including t2DM (Stivers, 2002). This Appalachian region is in need of targeted interventions that address healthy eating to curb both the obesity and $\mathrm{t} 2 \mathrm{DM}$ epidemics.

This nurse-led pilot study used the MyPlate education tool- a simple, evidencebased, easily accessible, and free tool in a vulnerable, obese, Appalachian population, who are at high-risk or who have t2DM. Compared to other healthy eating methods (carbohydrate counting, fat gram counting, food pyramid and exchange diet), this type of healthy eating instruction takes less time (approximately $40 \%$ reduction in teaching time) and is easily understood, as the visual graphic helps clients identify serving size and healthy food options.

\section{Educator and Nursing Student Response to using the MyPlate Tool}

The nurse educators thought that the MyPlate tool, compared to other healthy eating teaching methods, reduced teaching time, encouraged individual, family and group discussion and interactions, and focused on positive behavior change versus focusing on negative behavior. In addition, the tool fostered interaction between the educator and the client, especially with food models, paper plates, and tool evaluation assessment. Educators stated that the MyPlate tool was not as complicated as other methods, for example, the exchange diet, food pyramid, carbohydrate counting, or the glycemic index. Peer reviewed literature has demonstrated that shorter interventions allow participants to remember and better synthesize health and diabetes education information. According to Menet, et al, (2010) shorter educational interventions are more effective than longer 
interventions and that shorter interventions allow participants to remember and better synthesize client education information. These observations are important, as the nurses are readily available in these rural clinics and this type of education can be implemented independent of the registered dietician.

The Cochrane Review by Deakin, McShane, Cade and Williams (2009), which evaluated group diabetes education programs demonstrated that there is no evidence to suggest that teaching is more effective if delivered by a physician, dietitian or nurse as long as the health professional is trained to deliver diabetes education. Programs based on therapeutic client education using the principles of empowerment, participation and adult learning proved to be effective. The MyPlate tool is proving to be simple and easy to comprehend, when instruction is provided by nurses and nursing students.

\section{Participant Survey Response}

The $53 \%$ survey return in this study is well above the national average of mailed survey responses (Battaglia, Khare, Frankel, Murray, Buckley \& Peritz, 2008). For example, the Behavioral Risk Factor Surveillance Survey has seen a response rate decline of one-half to one percentage point each year, with a survey return between 30-40 percent (Kreuter, 2013). This study survey return is particularly impressive given the time between the one-time intervention and survey follow-up, and the fact that this vulnerable population is affected by frequent housing relocation.

\section{Baseline Survey Results}

The baseline, point of care survey results demonstrated that the responders said that the MyPlate tool was easy to comprehend. The ease of comprehension and reduced 
teaching time can encourage nurses who have previously not included healthy eating instruction in their practice to consider including it when educating clients.

The high percentage of clients who said their family members could follow the MyPlate tool indicates that this type of eating is likely to be accepted by family members, this could reduce the clients' feelings of isolation related to healthy eating. Family acceptance and support of a behavior change is critical for client success. Having family buy-in benefits not only the client, but the family too. According to Tyler \& Horner (2008), family centered care and health care practices are learned in the family and health promotion change is improved by family support.

The majority of responders said they could follow this tool when eating at home, and slightly less, but still a majority, said they could follow this when eating out. This has teaching implications for nurses working with this population. Client instruction should address the differences between food selections inside and outside the home. Examples of healthy choices when eating outside the home should be included in the teaching.

Compared to other diet methods, the majority of the participants in this study stated that the MyPlate tool was easier to comprehend compared to other diet methods. In line with Nau (2012), less complex diabetes treatment regimens are associated with higher rates of adherence to diabetes treatment programs. Serono et al (2007) suggested that educational tools geared towards those with diabetes or those at risk of developing diabetes and who reside in medically underserved areas, need to be simple, culturally appropriate, focusing on improving dietary and physical activity behaviors.

The results of the MyPlate baseline survey demonstrated that the tool was seen as easy to understand and that the clients thought they could follow these food 
recommendations at home and when eating outside the home. Levine, et al, evaluated the MyPlate communication campaign and made two recommendations: focus evaluation efforts in understanding the effectiveness of the MyPlate, and evaluate strategies to increase the effectiveness of Dietary Guidelines in vulnerable populations (2012). With this improved client comprehension, nurses working with this population should incorporate this simple teaching tool into their nursing plan of care.

\section{Outcome Results}

This study demonstrated several documented trends, albeit not statistically significant. These trends have clinical significance in vulnerable, high-risk individuals who are at risk of developing t2DM due to excess body weight.

\section{Weight loss.}

Comparisons made for responders at baseline and follow-up demonstrated that weight loss did occur in this population, who received no additional weight loss instruction since the initial MyPlate teaching. Considering that weight gain is normally gradual and continuous in obese populations, the mean weight loss is encouraging. Even though the 4.4 pound weight loss in the responders in this pilot study was not statistically significant, it does demonstrate a weight trend in a healthy direction. The majority of the responders $(53.1 \% \mathrm{n}=17)$ lost weight.

An analysis by Sheehan, et al, notes that the mean yearly weight gain for American women and men aged $36-47$ is $0.23 \mathrm{~kg}$ per year, which is equal to 0.5 pound (2003). If Appalachians improve their BMI, they would benefit from a gain of 1.5 (male) and 1.4 (female) additional life years (2010). An elevated BMI has an impact on cardiovascular wellness, development of t2DM and other chronic diseases. A reduction in 
BMI not only reduces the development of deadly diseases, but also extends life (Danaei, et al, 2010). The weight loss seen in this study is trending in a healthy direction, which is impressive, considering that the evidence suggests that, given this population's age and geographical location, they would be expected to have experienced a weight gain of 0.5 pounds.

\section{Gender difference.}

Women who were taught the MyPlate method lost more weight than men, although not statistically significant. Gender difference in healthy eating has been documented in the literature. For example, Denham, et al (2004) studied focus groups to determine how Appalachian women engage family members in health-promotion activities. The research demonstrated that family was one of the most important themes and that women take an active role in maintaining health among the individual family members. Whittemore (2004) reports that women who participated in diet education interventions demonstrated significantly better diet self-management $(\mathrm{P}=0.02)$ compared to men. The weight change between men and women in this MyPlate study may be related to female family member's familiarity with food preparation, as many women in this Appalachian region are the primary food preparers for the household. A study by Wansink \& Kranz noted that women, particularly mothers, are early adapters to the MyPlate (2013). Further MyPlate teaching strategies in this population could include targeted messages related specifically to women as food preparers and food purchasers. By tailoring the education interventions towards women, healthier food choices may impact all family members. 


\section{MyPlate visibility and weight loss.}

The location of the MyPlate tool in the home had an impact on weight loss; if the tool was seen daily, the weight trended towards a greater loss. A study by Levetan, et al, demonstrated that a visual educational tool placed in a location that was seen daily improved diabetes control (2002). The importance of tool visibility incorporates concepts of the HPM, in reinforcing positive behavior change by keeping "health benefits in front of the client as a reminder that the behaviors in the plan are personally worthwhile and directed toward important life goals" (Pender, et al, 2011, p. 129).

Further analysis was done on MyPlate visibility and weight change by categories: lost five or more pounds, stable within five pounds, or gained five or more pounds. Most of the participants who had their MyPlate tool visible on a daily basis lost five or more pounds. Although this is not statistically significant, the Cramers' V indicates a moderate effect size, which has implications for nursing practice. Further studies are needed to determine if the MyPlate tool location has an impact on weight loss and behavior change. Making the educational tools visible is easy, simple and free. This effect size could have important clinical implications for this region and population and other vulnerable populations.

Based on this preliminary result, further study related to daily visual reminders, would be beneficial. The literature review documents that visible educational tools have been proven effective and are a simple means of incorporating the HPM into nursing practice. Examples of integrating visual MyPlate tools into daily practice include: the MyPlate smart phone App, daily texts, tweets or social media messages, poster placement 
and table placemats. These simple tool placement interventions may have important clinical significance.

\section{MyPlate by meal.}

At baseline, participants believed that MyPlate could be followed for all meals. One year after instruction, the ability to follow MyPlate by meal was less than what was anticipated by the participants. Breakfast is the most challenging meal to follow the recommendations for proper nutrient distribution. Future teaching interventions should incorporate strategies that include breakfast balanced plates; for example, graphics should be used to show a healthy balanced breakfast plate, and breakfast food models. Specific breakfast items should be assessed by the nurse. Clients should be provided with written examples of MyPlate breakfast foods. Use of these visual reminders is supported by the HPM recommendation to develop a behavior-change plan.

\section{Portion Size.}

The ability of each client to follow the MyPlate portion size was assessed at baseline and follow-up. Correct portion size improved in the follow-up evaluation. This is an improvement, since food choices and portion size affect weight gain. Even though a higher percentage of responders said they followed MyPlate portion size at follow-up, a higher percentage said they follow MyPlate portion size some of the time or never at follow-up, compared to baseline. It must be noted that the survey questions relating to portion size varied from baseline to follow-up. For the baseline survey, a Likert scale was used and this appeared to confuse many participants. This baseline Likert scale was replaced in the follow-up survey with a check-off list. The change from a Likert scale to 
list check-off may have impacted the results and account for the discrepancy and inconsistencies in reported portion control.

The participant perception that MyPlate could be followed more than it actually was is an important finding that should be incorporated into nurse's MyPlate teaching plan. The nurse can offer teaching strategies by further incorporating the HPM into practice. For example, the teaching plan could include a formalized commitment to the behavior-change plan by developing written goals with the client that pertains to portion size.

\section{Barriers to following MyPlate.}

The post survey results, participants reported two barriers to following the MyPlate: cost and a big change in eating habits. The HPM suggests that perceived barriers are important determinants of health-promoting behavior. The responses to the follow-up survey are congruent with the HPM's perceived barriers. Understanding these barriers can help the nurse tailor participant education and encourage dialogue to address perceived barriers to healthy eating, with a focus on solutions. Examples include: low cost fruits, vegetables, whole grains, low fat protein sources and gradual incorporation of healthy foods into the diet to encourage long-term behavior change.

Using the HPM to guide the MyPlate intervention encouraged educators to tailor the MyPlate materials to the individual, by addressing favorite foods, individual eating habits, food preparation and family involvement. Future interventions can address these potential barriers during the nursing assessment and guide the discussion towards overcoming these barriers to healthy eating. Other vulnerable populations may experience similar barriers to following MyPlate. Nurses can incorporate specific 
examples of cost savings, including inexpensive food options, substitutes for higher cost foods can be taught during client education sessions.

\section{Theory-based Nursing Practice}

This MyPlate teaching project incorporated Pender's Health Promotion Model, which focuses on improving a client's well-being (Pender, et al, 2010). Ho, et al, (2010) identified four themes of the HPM that influence client empowerment including: trust in a nurse's competence, striving for client control, a desire for shared experiences, and an ability to personalize the material. Each nurse educator and student nurse incorporated the four themes identified by Ho with each client interaction. Trust was developed through the targeted nursing assessment and the use of individualized healthy eating interventions. Clients were given control of their behavior change by incorporating what they viewed as healthy eating priorities and writing these as goals that were given to the client, which personalized the teaching material for them.

The HPM model was used for this pilot study to guide assessment and interventions because it appreciates the current health level of each client, while guiding the client towards healthy choices. The model's commitment to a plan of action, as it relates to a commitment to carry out a specific action at a given time and place (in this case, eating) and the identification of strategies for carrying out the behavior, make it particularly appropriate with this vulnerable population. The Health Promotion Model's focus on improved wellness through positive behavior change, and the use of motivational interviewing, which has also proven effective in eliciting healthy behaviors, were used by the nurse educators and student nurses to guide the individualized and 
targeted nursing assessment and teaching MyPlate intervention for the clients at the RAM events.

The people of Appalachia, as previously documented, practice many poor health choices and both the Health Promotion Model and motivational interviewing focus on positive changes that can be worked into the client's routine rather than focusing on the negative behavior. The identified perceived barriers to following MyPlate will help target nursing and educational interventions in future MyPlate client instruction sessions.

\section{Participant Perceptions}

The perceived ability to follow MyPlate by meal and the actual ability to follow by meal was assessed and found that MyPlate was more challenging to follow than anticipated. Likewise, at follow-up $81 \%$ of the responders said they had lost weight however actual weight loss showed that $57 \%$ of the responders actually lost weight.

Participants were more optimistic about their perceived ability to follow the treatment plan than their actual ability to follow it. And, participants thought they lost more weight than they actually did. In future education sessions about My Plate, nurses should assess a client's ability to follow the MyPlate, but understand that a client's belief and desire to change behavior may be more optimistic than reality. Nurses can target the teaching interventions with these perceived beliefs in mind, as the client may become discouraged if their progress towards their healthy eating goal is not met. Incorporating concepts of the HPM into targeted interventions for a behavior change plan, allows the nurse and client to work towards realistic and achievable goals. 


\section{Study Benefits}

The nursing implications are far-reaching, as this one-time teaching tool was used with a vulnerable, obese population who is at risk of developing or worsening their t2DM. Due to its ease of use and easy accessibility, the MyPlate teaching tool can be adapted to a variety of client education environments. Analysis of these results can be used to guide future teaching interventions at the RAM events. The "train the trainer" model used with the nurse educators and student nurses can be adapted for lay educators and health promoters. The reduced client instruction time can be mimicked in other health care settings where nutritionists and nurse educators are not available. The MyPlate tool could be implemented in non-traditional health education settings, for example, telemedicine. This tool may be effective in similar economically disadvantaged client groups who are obese or at risk of developing t2DM.

The literature review noted that there were very few educational intervention studies that identified a nursing theory as a guide to the client intervention. This MyPlate teaching tool intervention is based on Pender's Health Promotion Model, which focuses on the client's desire to change behavior, versus scare tactics used in other health models. This theory-based educational assessment and intervention model can help nurses use a nursing theory to guide assessment and evidence-based practice interventions. By following this model, barriers to learning and health behavior change was identified and addressed at baseline and further assessed at follow-up. Interpersonal and situational influences were also assessed at baseline and individualized nursing interventions were employed with the client. 
Considerations for future MyPlate process evaluation could include: telephone follow-up, mailed information follow-up, telemedicine communication, MyPlate social media connections (twitter, Facebook or Pinterest), and client follow-up with the Health Wagon staff.

\section{Study Limitations and Lessons Learned}

One limitation is the convenience sample, which may show an under or over representation of the population. A second limitation related to the "stated weight" versus an actual weight in the follow-up survey. According to Shapiro \& Anderson (2003), selfreported weights are an accurate measure of true weight. A third limitation is the written survey; the respondent may not understand a question in the survey. This was addressed by re-wording the follow-up survey from the baseline survey, for example, the Likert scale was confusing for some respondents, so this was removed from the follow-up survey. Only forty-six of the original eighty-seven responders identified having a chronic disease on the survey and this was not verified by the nurses or students. For this reason, this question was repeated in the follow-up survey. Responders at baseline and follow-up may have omitted this question due to confusion, fatigue, or the respondent may have felt it was private information they did not want to share on the survey. A fourth limitation is the lack of race data collected at baseline. Based on the U.S. Census data (2012), each of the Virginia counties represented at the RM. clinics consists of a Caucasian population great than 93\% (Wise County 93.4\%, Lee County 94.7\%, Scott County 98.2\%, Dickenson County 98.8\%, Russell County 98\%, and Washington County 97.1\%). Demographic information collected during the 2008 Remote Area Medical clinic noted 
that of the 2,831 clients seen, 95.7\% were Caucasian (Snyder \& Milbrath, 2013). Finally, the small sample size could have impacted significance.

\section{Nursing Practice Implications}

The results of this pilot study have implications for an immediate and impressive impact on nursing practice. As health educators, nurses are often the first health care professionals that deal with clients on an intimate level. The effectiveness of the MyPlate tool is far reaching, as there are limited health care providers in this Appalachian region. The public has a great deal of trust in nurses. The evidence demonstrates that both obesity and $\mathrm{t} 2 \mathrm{DM}$ in the Appalachian region leads to shorter life expectancy and chronic, painful and expensive diseases. Nurses are well equipped with knowledge, assessment and communication skills to educate clients and communities on healthy eating.

In order to more effectively control the obesity and t2DM epidemic in Appalachia, more aggressive educational campaigns must be initiated in the region related to the prevention and management of the disease. In particular, individuals who are at high-risk of developing t2DM or are newly diagnosed are prime candidates for education about lifestyle modification, especially healthy nutrition choices. Studies have shown that people with pre-diabetes who lose weight and increase their physical activity can prevent or delay type 2 diabetes (The DPP group, 2002). This approach has the potential to significantly increase their quality of life. Education must appeal directly to the targeted population and take into account low health literacy and the Appalachian culture and norms.

For the RAM event, the nurse educators are RNs and nursing students, not nutritionists. Streamlining client education to this vulnerable and chronically ill 
population is beneficial not only to the client, but also to the volunteer staff, as it allows for faster, more consistent, evidence-based, and individualized nutrition education. Another benefit is that the analysis can guide future teaching methods that can be incorporated into the RAM events and the Health Wagon.

Nurses who live and work in the Appalachian region have a better understanding of the cultural nuances that pertain to eating. By using the MyPlate teaching tools and incorporating the health promotion model and motivational interviewing, nurses in this region are poised to offer healthy eating instruction that is more likely to be followed by the clients, as the nurse is a trusted, reliable resource.

The MyPlate intervention that was evaluated here used the HPM to guide assessment and interventions. Using the HPM model to develop targeted nursing assessment and interventions that focus on positive behavior change by empowering clients to make healthy, realistic and achievable goals, can help nurses in a variety of health care settings develop a health promotion plan for clients or the community. These steps are easily adaptable in many clinical settings; including hospitals, clinics, parish nursing, public health departments, community health centers, free clinics and schools. Nurses can and should develop interventions that address preventative measures related to the chronic disease and obesity epidemic. Future studies are needed to replicate this study in larger samples and in a variety of health care settings.

There were several results that can be immediately incorporated into client education to effectively improve healthy eating. First, all clients should be encouraged to place their educational materials in a location that will be viewed each day. Second, the role of the female as food preparer and family caregiver should be addressed when 
teaching all clients, particularly females. Specific suggestions for MyPlate food options at all meals should be addressed, particularly breakfast. Portion size, perceived and actual, should be discussed to guide clients in meeting their goals. And lastly, barriers to healthy food choices should be addressed in all MyPlate teaching sessions, as these barriers have an impact on client success.

\section{Sustainability}

This pilot study demonstrated that the MyPlate educational tool was effective in changing behavior by improving eating habits that led to weight loss. Sustainability efforts for the RAM participants will include educating the nurses and nursing students on these findings and incorporating these findings into tailored education interventions. These findings will also be summarized for the Health Wagon staff, including providers, nurses, community outreach workers and the tele-medicine diabetes staff at the University of Virginia Medical Center.

\section{Implications for Further Nursing Research and Development}

This nursing intervention, which tested an available teaching tool that was simple and was developed using evidence-based guidelines and practice, demonstrated positive results in a vulnerable, obese population at risk for T2DM. This pilot study demonstrated several clinically significant trends which should be further studied. Further nursing research is needed to test this tool in other vulnerable populations, including but not limited to: clients with English as a second language, Medicare and Medicaid recipients, recipients of Women Infant and Children (WIC), free clinics, and urban community health centers. 
The partnership between the nurse educators and nursing students needs to be further studied and encouraged. A recent interprofessional student service learning project on preventive care with an Appalachian population demonstrated that the program created clinical opportunities for students to practice collaboratively while assisting clients (Lee, Hayes, McConnell, Henry, 2013). This type of student professional partnership should be further tested and encouraged.

Another future area of research should include a comparison using the MyPlate teaching tool in a variety of teaching environments: comparing traditional face to face instruction to new methods of communication, for example, tele-medicine, skype and peer educators. Gender differences and MyPlate use should be further assessed and analyzed and specific gender education interventions should be tailored to the results. The differences in perceived and actual portion size and meal food distribution should be further evaluated and teaching interventions should be tailored specifically towards improving actual portion size and meal distribution.

This pilot study evaluation was done one year post intervention. Future MyPlate study should evaluate the intervention at three, six and 12 months post instruction. Various amounts of weight loss may be seen at these different stages.

Using a nursing theory to guide health promotion assessment and interventions should be evaluated on a larger scale. Using a nursing-based theory helps the profession identify health promotion goals that strengthen the bond between clients and nurses by empowering the clients to make healthy decisions. This theory integration can be used with many education programs affecting those with chronic disease management, where the day-to-day steps towards health promotion may prove overwhelming for the client. 
The results of this pilot study should guide further program evaluation and research in vulnerable populations at risk for chronic disease and obesity. The small sample size had an impact on statistical significance. A study design with a larger population should be a future consideration. Another consideration is chronic disease management and MyPlate instruction, including but not limited to diabetes, hypertension, heart disease and obesity. This pilot study evaluated only one intervention related to diabetes prevention: healthy eating. The DPP (2002) demonstrated that a combination of healthy eating and physical activity led to a $7 \%$ weight loss that reduced the chances of developing T2DM. Further study should evaluate a teaching program that incorporates both healthy eating with daily activity.

\section{Products}

The completed manuscript will be submitted to the American Journal of Nursing, as this is a simple nursing intervention that may benefit a variety of client populations. In addition, the reduction in one-on-one nursing instruction time and potential for family involvement is very relevant for nurses and the clients they help (Appendix W). The general nursing professional can benefit from this teaching tool and the results of a onetime education intervention in a vulnerable population.

In addition to the general nursing population, this simple MyPlate tool may be useful for health care providers in rural areas to improve client knowledge and health practices. A second method of disseminating these findings is through professional presentations geared towards professionals who work with vulnerable populations. A professional podium symposium presentation in June, 2013, highlighted the educational intervention and nursing student involvement at the combined annual meeting of The 
Association of Community Health Nursing Educators (ACHNE) and the Association of Public Health Nurses (APHN). Further presentations, summarizing the results of the baseline and follow-up surveys are planned in 2014. Targeted groups include the American Public Health Association, the American Association of Diabetes Educators, and Rural Nursing. 


\section{References}

Ackermann, RT, Finch EA, Brizendine E, Honghong Z, Marrero DG. (2008). Translating the diabetes prevention program into the community The DEPLOY pilot study. Am J Prev Med 35(4), 357-363. Doi:10.1016

American Association of Diabetes Educators, AADE 7 Self Care Behaviors (2012).

Retrieved from:

http://www.diabeteseducator.org/ProfessionalResources/AADE7/

American Diabetes Association (2011). Retrieved from: http://www.diabetes.org/

American Diabetes Association (ADA). (2008). Position Statement: Economic Costs of Diabetes in the U.S. in 2007. Diabetes Care, 31 (3), 1-20.

American Diabetes Association (2012). Position Statement: Standards of Medical Care in Diabetes-2012 Diabetes Care, 35, S11-S63; doi:10.2337/dc12-s011

American Diabetes Association (2004). Prevention or delay of type 2 diabetes. Diabetes Care 27 (Suppl 1). S47-S54.

American Dietetic Association (2006). Evidence based library. ADA Evidence Library Web site.ADA Evidence Library Web site. http://www.adaevidencelibrary.com

Anderson, R. M., \& Funnell, M. M. (2010). Patient empowerment: Myths and misconceptions. Patient Education and Counseling, 79(3), 277-282. doi: 10.1016/j.pec.2009.07.025

Appalachian Regional Commission (2006-2011) (2012). Retrieved from: www.arc.gov/ Balamurugan, A, Ohsfeldt, R, Hughes, T, Phillips, M. (2006). Diabetes self-management education program for Medicaid recipients: a continuous quality improvement process. Diabetes Eduator; 32, 893-900. 
Barker L, Crespo R, Gerzoff RB, Denham S, Shrewsberry M, Cornelius-Averhart D.(2010). Residence in a distressed county in Appalachia as a risk factor for diabetes, Behavioral Risk Factor Surveillance System, 2006-2007. Prev Chronic Dis 7(5),1-9. Retrieved from: http://www.cdc.gov/PCD/issues/2010/sep/pdf/09_0203.pdf

Battaglia,M.P., Khare, M., Frankel, M.R., Murray,M.C., Buckley, P. \& Peritz,S.(2008). Response rates: How have they changed and where are they headed? In Wiley, J.(Ed.), Advances in telephone survey methodology.

DOI: 10.1002/9780470173404.ch24. Retrieved from: http://onlinelibrary.wiley.com.proxy.its.virginia.edu/doi/10.1002/9780470173404. $\operatorname{ch} 24 /$ summary

Beckles GL, Engelgau MM, Narayan KM, et al (1998). Population based assessment of the level of care among adults with diabetes in the U.S. Diabetes Care,21, 14328.Behavioral Risk Factor Surveillance System.(2010). Retrieved from: http://www.cdc.gov/brfss/

Behringer, B., \& Friedell, G. H. (2006). Appalachia: Where place matters in health. Preventing Chronic Disease, 3(4), 1-4. http://www.ncbi.nlm.nih.gov/pmc/articles/PMC1779277/pdf/PCD34A113.pdf

Birdsall S, Florin J. (2008) An outline of American geography. Chapter 7: Appalachia and the Ozarks. US Department of State.

Bobroff, L. B., Minton, E., Diehl, D. C., Diaz, X., Keith, M., Medina-Solorzano, A., \& Gylfadottir, U. K. (2012). Evaluation of MyPlate mini-poster for older latino adults: MiPlato para adultos mayores. Faseb Journal, 26 
Boddiger, D. (2006). Lack of insurance hinders Americans' diabetes care. Lancet, 368(9529), 15-16.

Boren, S., Fitzner, K., Panhalkar, P., \& Specker, J. (2009). Costs and benefits associated with diabetes education: a review of the literature. Diabetes Educator, 35(1), 72 96.doi:10.1177/0145721708326774

Brobeck E, Bergh H, Odencrants S, Hildinghusing, C. (2011). Primary healthcare nurses' experiences with motivational interviewing in health promotion practice. Journal of Clinical Nursing: 20, 3322-3330.

Brown, AF, Ettner, SL, Piette, J, Weiberger, M, Gregg, E, Shapiro, MF, Karter, AJ, Safford, M., Waitzfelder, B, Prata, PA, Beckles, GL. (2004). Socioeconomic position and health among persons with type 2 diabetes: A conceptual model and Review of the literature. Epidemiological Reviews, 26, 63-

77.doi:10.1093/epirev/mxh002

Bybee, R., \& Thompson, S. (2004). An innovative program to fund health-oriented student projects and research. Journal of American College Health, 53(2), 85-87.

Centers for Disease Control and Prevention (2011). National diabetes fact sheet: national estimates and general information on diabetes and prediabetes in the United States. Atlanta, GA: U.S. Department of Health and Human Services, Centers for Disease Control and Prevention.

Centers for Disease Control, Diabetes Prevalence Data, retrieved from: http://apps.nccd.cdc.gov/DDT_STRS2/CountyPrevalenceData.aspx?stateId=51\& Mode $=$ DBT 
Concha, J., Kravitz, H., Chin, M., Kelley, M., Chavez, N., \& Johnson, T. (2009).

Perspectives in practice. Review of type 2 diabetes management interventions for addressing emotional well-being in Latinos. Diabetes Educator, 35(6), 941-958. doi:10.1177/0145721709344124

Coyne, C. , Demian-Popescu,C., Friend, D. (2006). Social and Cultural Factors Influencing Health in Southwestern West Virginia: A qualitative study. Prev Chronic Dis, 3(4), 1-8.

Crandall JP, Knowler, W.C., Kahn, S.E., S.E., Marrero, D., Florez, J.C., Bray, G.A., Haffner, S.A., Hoskin, M. \& Nathan, D.M. (2008) The prevention of type 2 diabetes.Nat Clin Pract Endocrinol Metab, 4, 382-393.

Dabney, B. \& Gosschalk, A. (2010). Diabetes in rural America: A literature review. Rural Healthy People 2010. Retrieved from: http://srph.tamhsc.edu/centers/rhp2010/05Volume2diabetes.pdf

Danaei G, Rimm EB, Oza S, Kulkarni SC, Murray CJL, et al. (2010) The Promise of Prevention: The Effects of Four Preventable Risk Factors on National Life Expectancy and Life Expectancy Disparities by Race and County in the United States. PLoS Med 7(3): e1000248. doi:10.1371/journal.pmed.1000248

Deakin, McShane, Cade and Williams (2009). Group based training for self-management strategies in people with type 2 diabetes mellitus, Cochrane Database of Systematic Reviews, (2). DOI: 10.1002/14651858.CD003417.pub2

Della, L. (2011). Exploring diabetes beliefs in at-risk Appalachia. The Journal of Rural Health, 27(1), 3-12. doi: 10.1111/j.1748-0631.2010.00311.x Retrieved from: http://onlinelibrary.wiley.com/doi/10.1111/j.1748-0361.2010.00311.x/abstract 
Denham, S.A. (2003). Family health: A framework for nursing. Philadelphia, PA: F.A. Davis.

Denham, S. A., Manoogian, M. M., \& Schuster, L. (2007). Managing family support and dietary routines: Type 2 diabetes in rural appalachian families. Families, Systems, \& Health, 25(1), 36-52. doi: 10.1037/1091-7527.25.1.36

Denham, S., Meyer, M. \& Toborg, M. (2004). Tobacco cessation in adolescent females in Appalachian communities. Family \& Community Health, 27(2), 170-181.

Denham, S. A., Meyer, M. G., Toborg, M. A., \& Mande, M. J. (2004). Providing health education to appalachia populations. Holistic Nursing Practice, 18(6), 293-301.

"Diabetes in Appalachia" (2011). Retrieved from: http://www.diabetesfamily.net/

Dixon, J.B. (2010). The effect of obesity on health outcomes. Molecular Cell Endocrinology, 316(2),104-8.

DPP Program Group. (2002). Reduction in the incidence of type 2 diabetes with lifestyle intervention or metformin. New England Journal of Medicine, 346, 393-403.

Duke, S., Colagiuri, S., \& Colagiuri, R. (2009). Individual patient education for people with type 2 diabetes mellitus. Cochrane Database Of Systematic Reviews, (1), doi:10.1002/14651858.CD005268.pub2

Edwards, P., Roberts, I., Clarke, M., DiGuiseppi, C., Pratap, S., Wentz, R., \& Kwan, J. (2002) Increasing response rates to postal questionnaires: systematic review. $B M J, 327,1183-1192$. Retrieved from: http://www.bmj.com/content/324/7347/1183 
Finkelstein, E.A., Trogdon,J.G., Cohen, J.W., Dietz, W. (2009). Annual Medical Spending Attributable To Obesity: Payer-And Service-Specific Estimates. Health Affairs, 28(5), 822-831.

Flegal KM, Carroll MD, Ogden CL, Curtin LR. (2010). Prevalence and trends in obesity among U.S. adults, 1999-2008. JAMA,303(3), 235-241.

Funnell, M., Brown, T., Childs, B., Haas, L., Hosey, G., Jensen, B., \& Weiss, M. (2012). National standards for diabetes self-management education. Diabetes Care, $35 \mathrm{~S} 101-8$.

Gaede,P., Pedersen, O. (2005). Multi-targeted and aggressive treatment of patients with type 2 diabetes at high-risk: what are we waiting for? Hormone \& Metabolic Research, 37, Suppl 16-82.

Gallop Poll (2013). Retrieved from: http://www.gallup.com/poll/1654/honesty-ethicsprofessions.aspx

Graziano, J., \& Gross, C. (2009). The effects of isolated telephone interventions on glycemic control in type 2 diabetes: a literature review. Advances In Nursing Science, 32(3), E28- 41. doi:10.1097/ANS.0b013e3181b0d6d6

Gregg, EW, Geiss, LS, Saaddine J, et al (2001). Use of diabetes preventive care and complications risk in two African-American communities. Am J Prev Med, 21, 197-202.

Halverson, J. A, Ma, L., and Harner, E. J. (2004). An analysis of disparities in health status and access to health care in the Appalachian region. Washington DC: Appalachian Regional Commission. 
Hardin-Fanning, F. (2013). Adherence to a Mediterranean diet in a rural Appalachian food desert. Rural and Remote Health, 12(2293), 8-12.

Hawthorne, K., Robles, Y., Cannings-John, R., \& Edwards, AGK. (2008). Culturally appropriate health education for type 2 diabetes mellitus in ethnic minority groups. Cochrane Database Of Systematic Reviews, (3)

Healthy People 2020 (2012). Improving the Health of Americans: US Department of Health and Human Services. Retrieved from: http://healthypeople.gov/2020/topicsobjectives2020/objectiveslist.aspx?topicId=8

The Health Wagon (2011). Retrieved from http://www.thehealthwagon.org/clinics2.htm. Heisler, M. (2010). Different models to mobilize peer support to improve diabetes selfmanagement and clinical outcomes: evidence, logistics, evaluation considerations and needs for future research Family Practice 27(suppl 1), i23-i32 doi:10.1093/fampra/cmp003

Heithoff, K.A., Cuffel, B.J., Kennedy, S., Peters, J. (1997). The Association Between Body Mass and Health Care Expenditures. Clinical Therapy, 19(4):811-820.

Herman WH, Hoerger TJ, Brandle M, Hicks K., Sorensen S, Zhang P, Hamman RF, Ackermann RT, Englegau MM, Ratner RE., Diabetes Prevention Program Research Group. (2005). The cost-effectiveness of lifestyle modification or metformin in preventing type 2 diabetes in adults with impaired glucose tolerance. Ann Intern Med. 142, 323-332.

Hill, A., Guernsey De Zapien, J., Staten, L.K., McClelland, D.J., Moore-Monroy, M. Meister, J.S., Rebecca Garza, R., Elenes, J., Steinfelt, V., Tittelbaugh, I.,\& 
Whitmer, E. (2007) From Program to Policy: Expanding the Role of Community Coalitions, Prev Chronic Dis. October; 4(4), A103.

Ho, A., Berggren, I., \& Dahlborg-Lyckhage, E. (2010). Diabetes empowerment related to Pender's Health Promotion Model: a meta-synthesis. Nursing \& Health Sciences, 12(2), 259-267. doi:10.1111/j.1442-2018.2010.00517.x

Institute of Medicine. Dietary Reference Intakes for Energy, Carbohydrate, Fiber, Fat, Fatty Acids, Cholesterol, Protein, and Amino Acids. (2002).Washington (DC): The National Academies Press.

Jarvis, J., Skinner, TC, Carey, ME, Davies, MJ. (2010). How can structured selfmanagement patient education improve outcomes in people with type 2 diabetes? Diabetes, Obesity \& metabolism 12(1), 12-9.

Joy, S. (2008). Clinical Pearls and Strategies to Optimize Patient Outcomes The Diabetes Educator 34, 54S-59S, doi:10.1177/0145721708319233

Knowler, WC, Barrett-Conner E, Fowler SE, Hamman RF, Kachin JM, Walker EA, Nathan DM.Diabetes Prevention Program Research group (2002). Reduction in the incidence of type 2 diabetes with lifestyle intervention or metformin. $N$ Engl J Med; 346, 393-03.

Kosaka, K, Noda M, Kuzuya T., (2005). Prevention of type 2 diabetes by lifestyle intervention: a Japanese trial in IGT males. Diabetes Res Clin Pract; 67,152-162.

Kreuter, F. (2013) Facing the Nonresponse Challenge. The ANNALS of the American Academy of Political and Social Science January (645), 23-35, doi:10.1177/0002716212456815 
Retrieved from:

http://ann.sagepub.com.proxy.its.virginia.edu/content/645/1/23.full

Lee, M., L., Hayes, P., A., McConnell, P., \& Henry, R., M. (2013). Students delivering health care to a vulnerable Appalachian population through interprofessional service-learning.Gerontology \& Geriatrics Education, 34(1), 43-59. doi:10.1080/02701960.2013.737388

Levetan, C. Dawn, K., Robbins, D., Ratner, R. (2002) Impact of Computer-Generated Personalized Goals on HbA1c. Diabetes Care, 25(1), 2-8.

Levine, E., Abbatangelo-Gray, J., Mobley, A. R., McLaughlin, G. R., \& Herzog, J. (2012). Evaluating MyPlate: An expanded framework using traditional and nontraditional metrics for assessing health communication campaigns. Journal of Nutrition Education and Behavior, 44(4), S2-S12. doi:10.1016/j.jneb.2012.05.011

Loveman, E., Frampton, G.K. and Clegg, A.J. (2008). The clinical effectiveness of diabetes education models for Type 2 diabetes: a systematic review. Health Technology Assessment, 12, (9), 1-136. (oi:10.3310/hta12090). (MID:18405469). Miller,W., Rollnick, S. (2002). Motivational Interviewing, Second Edition: Preparing People for Change. Guilford Press, New York.

Minet, L., Møller, S., Vach, W., Wagner, L., \& Henriksen, J. (2010). Mediating the effect of self-care management intervention in type 2 diabetes: a meta-analysis of 47 randomised controlled trials. Patient Education \& Counseling, 80(1), 29-41. doi:10.1016/j.pec.2009.09.033

Morgan, L. L., \& Reel, S. J. (2003). Developing Cultural Competence in Rural Nursing. Online Journal of Rural Nursing and Health Care, (3)1, 28-37. 
National Certification Board for Diabetes Educators, (2012). http://www.ncbde.org/

National Diabetes Education Program (NDEP) (2009). Guiding Principles for Diabetes

Care: For Health Care Professionals. Available at:

http://ndep.nih.gov/media/GuidPrin_HC_Eng.pdf

Nau, DP. (2012). Recommendations for improving adherence to type 2 diabetes mellitus therapy-focus on optimizing oral and non-insulin therapies. American Journal of Managed Care. 18(3 Suppl), S49-54.

Nelson, KM., Reiber, G., Boyko, EJ. (2002). Diet and exercise among adults with type 2 diabetes: findings from the third national health and nutrition examination survey (NHANES III) Diabetes Care, 25, 1722-1728.

Norris, SL, Lau, J., Smith SJ, Schmid, DH, Engelgau, MM. (2002). Self-management education for adults with type 2 diabetes: a meta analysis of the effect on glycemic control. Diabetes Care; 25,1159-1171.

O'Connor, L.E., Brown, O., \& Savaiano, D.(2013) Text messaging enhances nutrition knowledge and behavior among college students: "Mobile MyPlate" FASEB Journal 27:626.2

Ogden CL, Carroll, MD, Curtin, LR, et al. (2006). Prevalence of overweight and obesity in the United States, 1999--2004. JAMA, 295, 1549-55.

Ogden, C. L., \& Carroll, M. D. (2010). Prevalence of overweight, obesity, and extreme obesity among adults: United States, trends 1960-1962 through 2007-2008. National Center for Health Statistics, 6, 1-6. 
Ogden CL, Caroll, MD, Kit, BK, Flegal, KM. (2012). Prevalence of Obesity and Trends in Body Mass Index Among US Children and Adolescents, 1999-2010. JAMA, 307(5),483-490. doi: 10.1001/jama.2012.40.

Pan, XR, Li GW, Hu YH, Wang JX, Yang WY, An ZX, Lin J, Siao JZ, Cao HB, Liu PA, Jiang, XG, Jiang YY, Wang JP, Zheng H, Zhang H, Bennett, PH, Howard BV. (1997). Effects of diet and exercise in preventing NIDDM in people with impaired glucose tolerance. The Da Zing IGT and Diabetes Study. Diabetes Care; $20,537-544$.

Pender, N. J., Murdaugh, C. L., \& Parsons, M. A. (1996). Health promotion in nursing practice. USA: Appleton \& Lange.

Pender NJ, Murdaugh CL, Parsons MA. (2006).Health Promotion in Nursing Practice (5th ed). Upper Saddle River, NJ: Prentice Hall.

Pender NJ, Murdaugh CL, Parsons MA. (2011). Health Promotion in Nursing Practice (6th ed). Upper Saddle River, NJ: Prentice Hall.

Post, R. C., Eder, J., Maniscalco, S., Johnson-Bailey, D., \& Bard, S. (2013). MyPlate is now reaching more consumers through social media. Journal of the Academy of Nutrition and Dietetics, 113(6), 754-755. doi:10.1016/j.jand.2013.04.014

RAM USA (2012). Available from: http://www.ramusa.org/

Ramachandran A, Snehalatha C, Mary S, Mukesh B, Bhaskar AD, Vijay V, Indian Diabetes Prevention Programme (IDPP) (2006). The Indian diabetes prevention programme shows that lifestyle modification can metformin prevent type 2 diabetes in Asian Indian subject with impaired glucose tolerance (IDPP-1). Diabetologia; 49, 289-297. 
Satterfield D, Volansky M, Caspersen C, Engelgau M, Bowman B, Gregg E, Geiss L, Hosey G, May J, Vinicor F (2003). Community-based lifestyle interventions to prevent type 2 diabetes. Diabetes Care 26, 2643-2652

Scollan-Koliopoulos, M., O'Connell, K., \& Walker, E. (2005). The first diabetes educator is the family: using illness representation to recognize a multigenerational legacy of diabetes. Clinical Nurse Specialist: The Journal For Advanced Nursing Practice, 19(6), 302-307.

Seidel MC, Powell RO, Zgibor JC, Siminerio LM, Piatt GA. (2008). Translating the Diabetes Prevention Program Into an Urban Medically Underserved Community: A nonrandomized prospective intervention study Diabetes Care, 31(4), 684-689.

Serrano, E., Leiferman, J., \& Dauber, S. (2007). Self-efficacy and health behaviors toward the prevention of diabetes among high-risk individuals living in Appalachia. Journal of Community Health, 32(2), 121-133. http://www.springerlink.com/content/2013v1q755313274/fulltext.pdf

Shapiro, J., Anderson, D. (2003). The effects of restraint, gender and body mass index on the accuracy of self-reported weight. Int J Eat Disor, 34,177-180.

Sheehan, T.J., DuBrava, S., DeChello, L.M., Fang, Z. (2003). Rates of weight change for black and white Americans over a twenty year period. International Journal of Obesity, 27,498-504.

Shi L, Starfield B. (2000). Primary care, income inequality, and self rated health in the United States: a mixed-level analysis. Int J Health Serv, 30,541-55. 
Sigurdardottir, A. K., Jonsdottir, H., \& Benediktsson, R. (2007). Outcomes of educational interventions in type 2 diabetes: WEKA data-mining analysis. Patient Education and Counseling, 67(1-2), 21-31. doi: 10.1016/j.pec.2007.03.007

Silva-Smith, A. L., Fleury, J., \& Belyea, M. (2013). Effects of a physical activity and healthy eating intervention to reduce stroke risk factors in older adults. Preventive Medicine, 57(5), 708-711.

doi:http://dx.doi.org.proxy.its.virginia.edu/10.1016/j.ypmed.2013.07.004

Snyder, A.E., Milbrath, G.R. (2013). Description of healthcare needs at an episodic clinic in rural southwest Virginia. Rural and Remote Health, 13, 2557. Retrieved from: http://www.rrh.org.au

Sohn M.F. (2005). Food origins: regional and cultural roots. In: Appalachian home cooking: history, culture and recipes. Lexington, KY: University of Kentucky Press.

Stivers C, Powell, T, Haussler, J, Forburger, AM. Diabetes in Virginia (2002). Available from: http://www.vahealth.org/diabetes/Diabetes_in_VA_2002.pdf

Sumlin, L.L., \& Garcia, A.A. (2012). Effects of Food-Related Interventions for African American Women with Type 2 Diabetes. Diabetes Educator, 38(2), 236-249. doi: $10.1177 / 0145721711422412$

Trust for Americans Health and the Robert Wood Johnson Foundation (2013). F as in Fat : How obesity Threatens America's future, 2013 report. Retrieved from: http://fasinfat.org/

Tuomilehto J, Linstrom J., Erisson JG, Valle TT, Hamalainen H, Ilanne-Parikka P, Keinanen-Kiukaanniemi S, Laakso M, Louheranta A, Rastas M, Salminen V, 
Uusitupa M, Finnish Diabetes Prevention Study Group (2001).Prevention of type 2 diabetes mellitus by changes in lifestyle among subjects with impaired glucose tolerance. N Engl J Med; 344,1343-1350.

Tyler, D. O., \& Horner, S. D. (2008). Family-centered collaborative negotiation: A model for facilitating behavior change in primary care. Journal of the American Academy of Nurse Practitioners, 20(4), 194-203. doi:10.1111/j.17457599.2007.00298.x

U.S. Census Bureau (2009). http://censtats.census.gov/cgibin/usac/usatable.pl?State $=\&$ County $=51195 \&$ TableID $=$ AAA

U.S Census Bureau (2012). http://quickfacts.census.gov/qfd/states/51/51195.html

U.S. Department of Agriculture and U.S. Department of Health and Human Services (2010). Dietary Guidelines for Americans, 2010. $7^{\text {th }}$ Edition, Washington, DC: U.S. Government Printing Office.

U.S. Department of Agriculture (2011). Center for Nutrition Policy and Promotion, MyPlate campaign. Retrieved from: http://www.choosemyplate.gov/index.html University of Virginia Health Systems (2012). Retrieved from: https://uvahealth.com/about/community/ram/ram-grundy

Urbanski, P., Wolf, A., \& Herman, W. (2008). Cost-effectiveness of diabetes education. Journal Of The American Dietetic Association, 108(4), S6-11.

Uruakpa, F. O., Moeckly, B. G., Fulford, L. D., Hollister, M. N., \& Kim, S. (2013). Awareness and use of MyPlate guidelines in making food choices. Procedia Food Science, 2(0), 180-186. doi:http://dx.doi.org.proxy.its.virginia.edu/10.1016/j.profoo.2013.04.026 
UVa today (2009). Retrieved from: http://www.news.virginia.edu/content/uva-healthsystem-planning-20-percent-increase-patients-seeking-care-ram-clinic

Van Dam, RM, Rimm, EB, Willet, WC, Stampfer, MJ, Hu FB. (2002). Dietary patterns and risk for type 2 diabetes mellitus in US men. Ann Intern Med: 136, 201-209.

Virginia Department of Health (2011). Appalachian region and diabetes. Retrieved from: http://www.vahealth.org

Virginia Department of Health (2011). Diabetes in Virginia. Richmond, VA: VDH, Division of Prevention and Health Promotion. Retrieved from: https://www.vdh.virginia.gov/ofhs/Prevention/diabetes/documents/2012/pdf/Diab etes\%20Burden\%20Report1.pdf

Virginia Diabetes Plan 2008-2017. Virginia Diabetes Council, www.virginiadiabetes.org Wansink, B., \& Kranz, S. (2013). Who's using MyPlate? Journal of Nutrition Education and Behavior, 45(6), 728-732. doi:10.1016/j.jneb.2013.03.009

Wens, J., Vermeire, E., Hearnshaw, H., Lindenmeyer, A., Biot, Y., \& Van Royen, P. (2008). Educational interventions aiming at improving adherence to treatment recommendations in type 2 diabetes: A sub-analysis of a systematic review of randomised controlled trials. Diabetes Research and Clinical Practice, 79(3), 377-388. doi: 10.1016/j.diabres.2007.06.006

Wewers, M., Ahijevych, K., Chen, M., Dresbach, S., Kihm, K., Kuun, P. (2000). Tobacco use characteristics among rural Ohio Appalachians. J. Community Health, 25(5), 377-88.

Whittemore R, Melkus GD, Sullivan A, Grey M. (2004). A nurse-coaching intervention for women with type 2 diabetes. Diabetes Educator; 30(5),795-804. 
Whittemore, R. (2006). Behavioral interventions for diabetes self-management. Nursing clinics of North America. 41(4),641-54.

Wolf A.M., Conaway M.R., Crowther J.Q., Hazen K.Y., Nadler J.L., Oneida B., Bovbjerg V.E (2004). Translating lifestyle intervention to practice in obese patients with type 2 diabetes: Improving Control with Activity and Nutrition (ICAN). Diabetes Care 27. 1570-1576.

Zabaleta, AM, Forbes, A. (2007). Structured group-based education for type 2 diabetes in primary care. British Journal of Community Nursing 12/(4), 158-162. 


\section{Appendix A}

Virginia - Percentage of Adults (aged 18 years or older) with Diabetes Who Are Overweight or Obese, By Age, 1995 - 2010

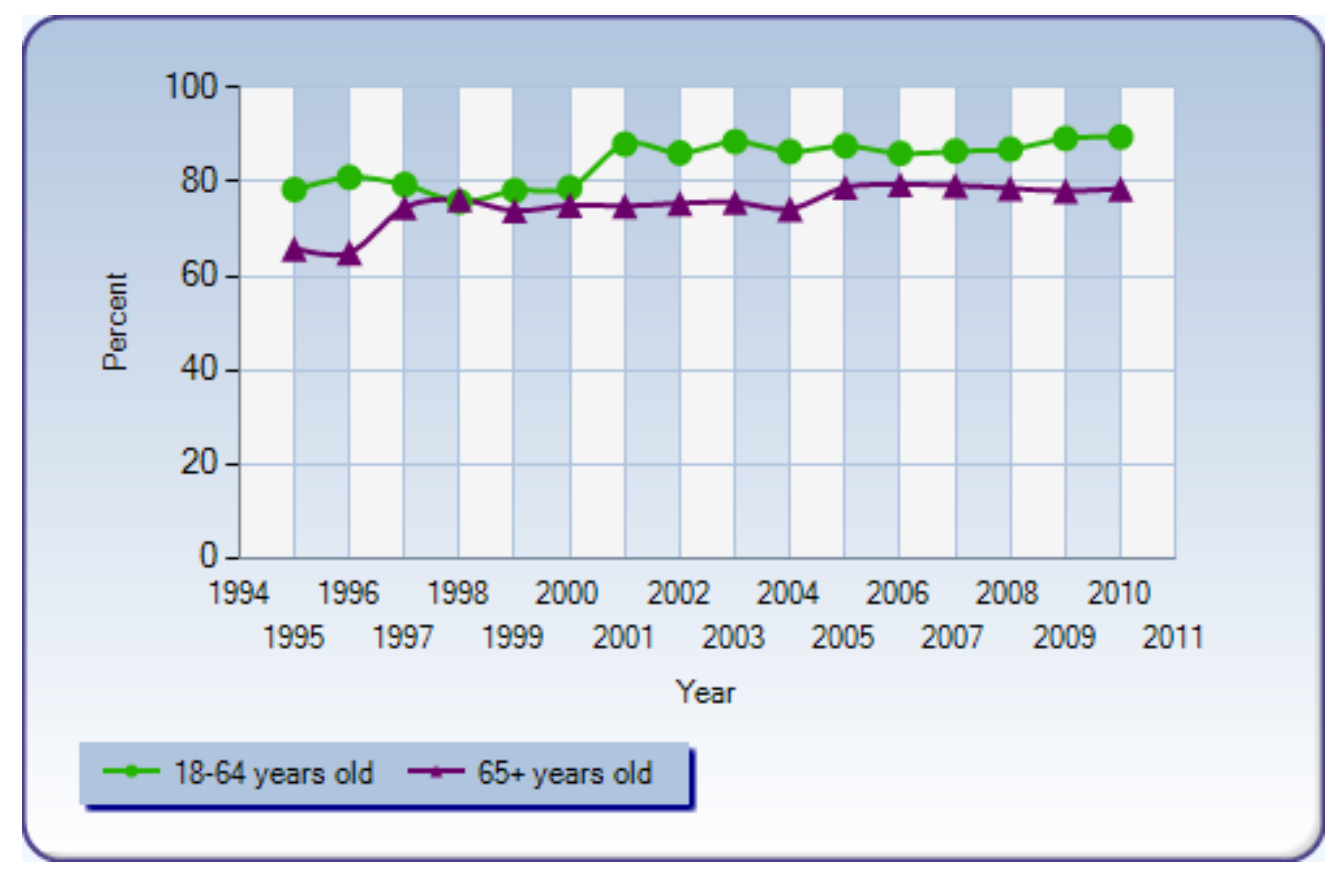

Retrieved from:

http://apps.nccd.cdc.gov/ddtstrs/Index.aspx?stateId=51\&state=Virginia\&cat=riskfactors $\&$ Data $=$ data $\&$ view $=$ TO $\&$ id $=21 \&$ trend $=$ overobese 


\section{Appendix B}

County Economic Status in Appalachia, FY 2014

(Effective October 1, 2013 through September 30, 2014)

The Appalachian Regional Commission uses an index-based county economic classification system to identify and monitor the economic status of Appalachian counties. See the methodology for a description of each economic level.

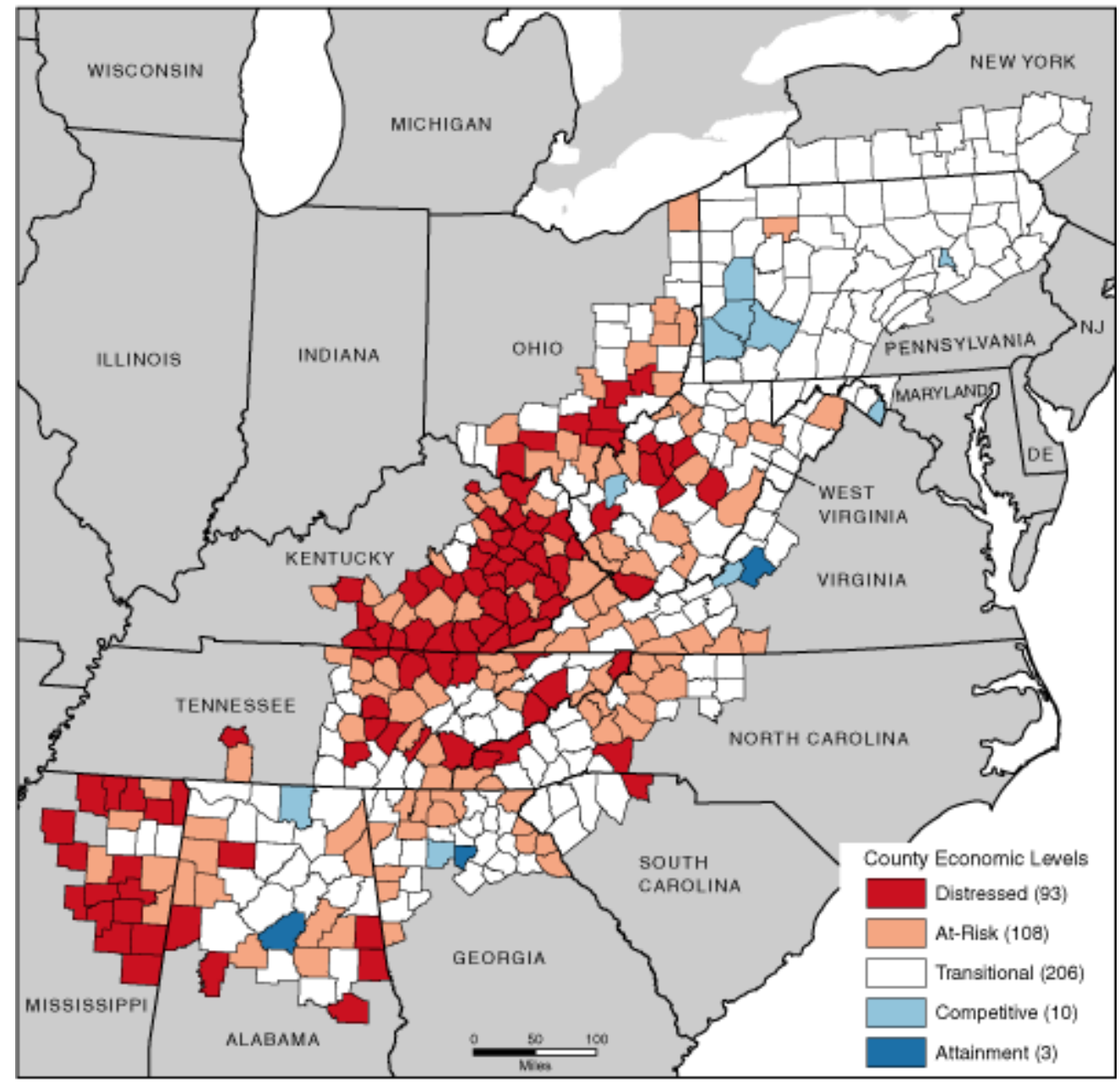

Created by the Appalachian Regional Commission, March 2013 Data Sources:

Unemployment data: U.S. Bureau of Labor Statistics, LAUS, 2009-2011

Income data: U.S. Bureau of Economic Analysis, REIS, 2010

Poverty data: U.S. Census Bureau, American Community Survey, 2007-2011

Effective October 1, 2013 through September 30, 2014

Accessed from: http://www.arc.gov/research/MapsofAppalachia.asp?MAP_ID=71 


\section{Appendix C}

Prevalence of Diabetes in Appalachian versus Non-Appalachian Counties of Virginia,

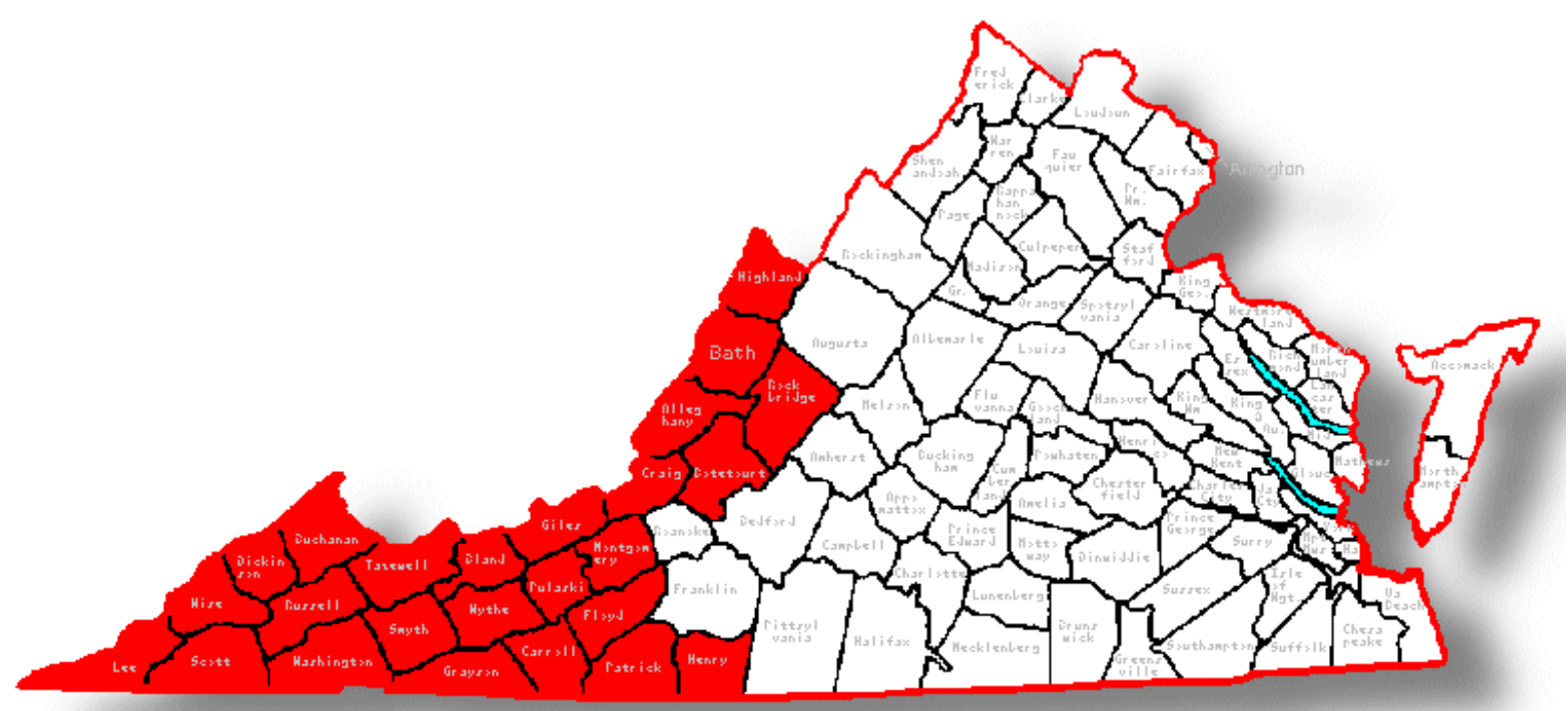

Non-Appalachian counties (in white): $7.4 \%$

Appalachian counties (in red): $13.9 \%$

Source: Appalachian Regional Commission: www.arc.gov/; Virginia Behavioral Risk Factor Surveillance System (BRFSS), 2009. 


\section{Appendix D}

2009 Age-Adjusted Estimates of the Percentage of Adults ${ }^{\dagger}$ Who Are Physically Inactive in Virginia
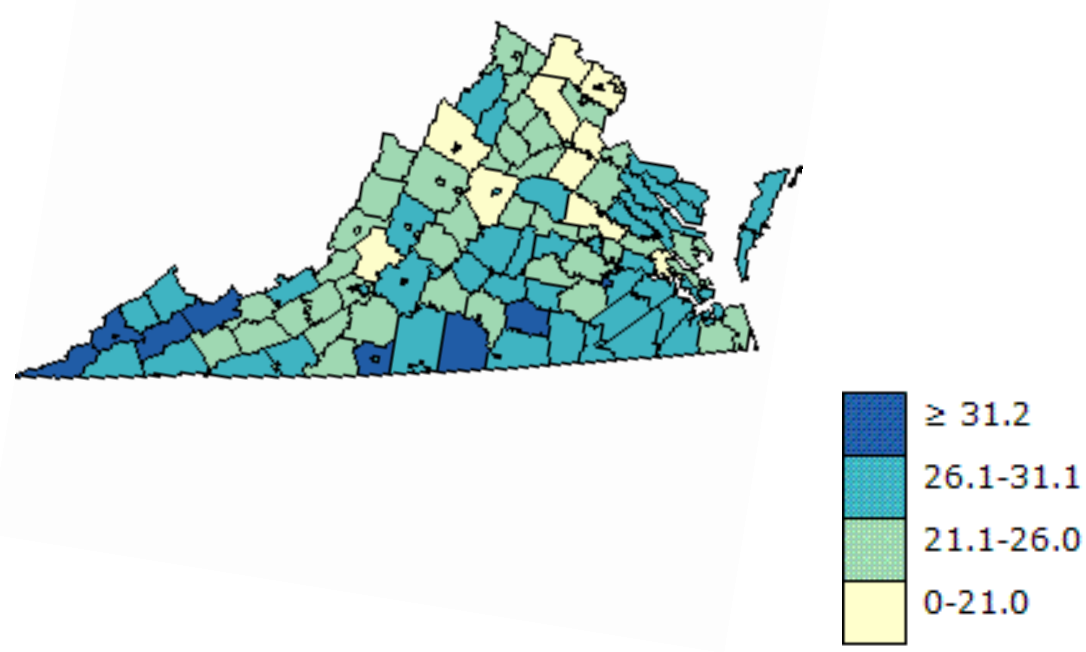

Retrieved from:

http://apps.nccd.cdc.gov/DDT_STRS2/CountyPrevalenceData.aspx $?$ stateId=51\&Mode=P HY 


\section{Appendix E}

2009 Age-Adjusted Estimates of the Percentage of Adults ${ }^{\dagger}$ Who Are Obese in Virginia
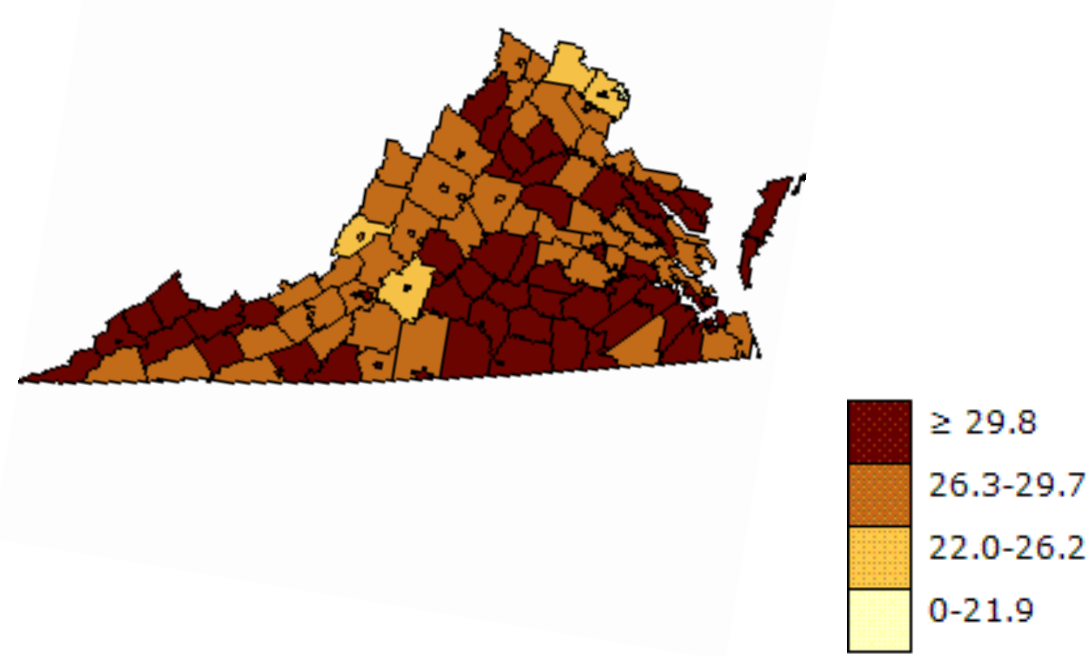

Retrieved from:

http://apps.nccd.cdc.gov/DDT_STRS2/CountyPrevalenceData.aspx stateId=51\&Mode= OBS 


\section{Appendix F}

Health Promotion Model (revised)

Pender, Murdaugh, Parsons, 2011

INDIVIDUAL CHARACTERISTICS AND EXPERIENCES

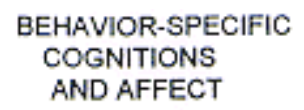

BEHAVIORAL OUTCOME

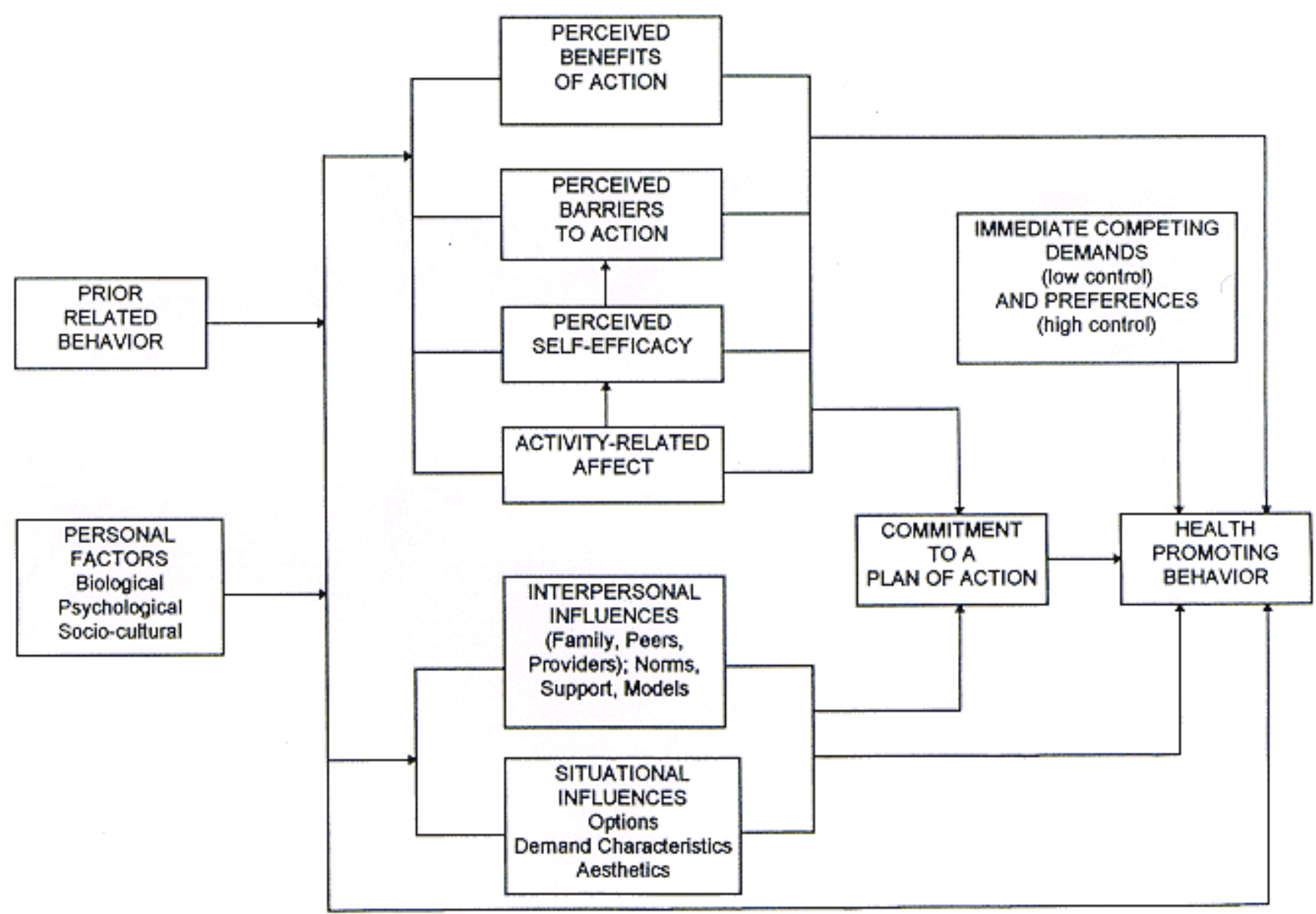

Revised Health Promotion Model

Source:

Pender NJ, Murdaugh CL, Parsons MA. (2011). Health Promotion in Nursing Practice

(6th ed). Upper Saddle River, NJ: Prentice Hall. p.45 


\section{Appendix G}

Table 1: Acceptable Macronutrient Distribution Ranges (AMDR)

\begin{tabular}{|l|r|r|r|}
\hline & Carbohydrate & Protein & Fat \\
\hline Young children (1-3 years) & $45-65 \%$ & $5-20 \%$ & $30-40 \%$ \\
\hline Older children and adolescents (4- & & & $25-35 \%$ \\
18 years) & $45-65 \%$ & $10-30 \%$ & $20-35 \%$ \\
\hline Adults (19 years and older) & $45-65 \%$ & $10-35 \%$ & \\
\hline
\end{tabular}

Source: Institute of Medicine. Dietary Reference Intakes for Energy, Carbohydrate,

Fiber, Fat, Fatty Acids, Cholesterol, Protein, and Amino Acids. Washington (DC): The National Academies Press; 2002. 
Appendix $\mathrm{H}$

MyPlate, U.S. Department of Agriculture, 2011

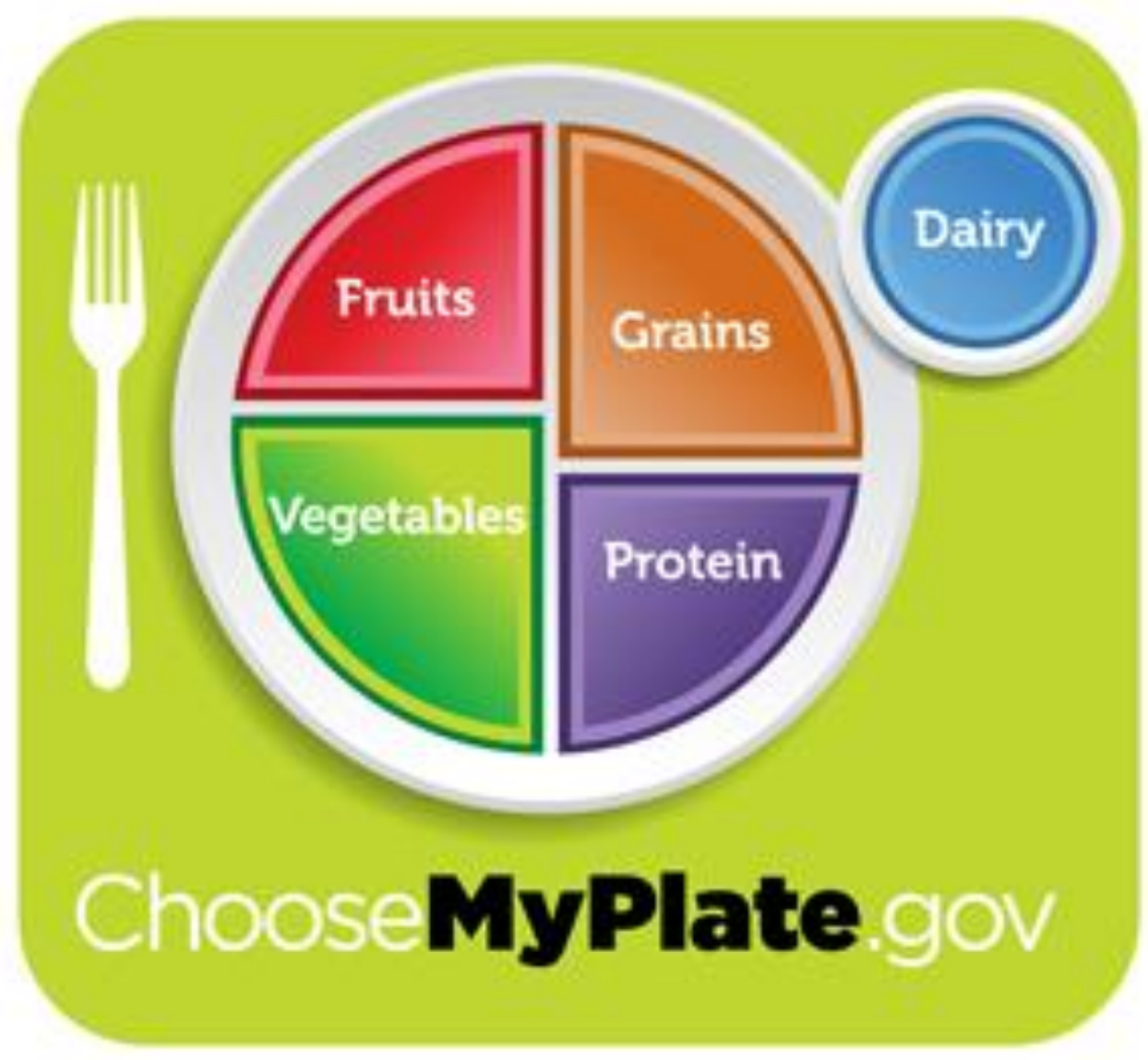

Retrieved from: www.myplate.gov 


\section{Appendix I}

Table 2. 2012 RAM Participants Seen at Both Medical Clinics.

\begin{tabular}{|l|c|c|}
\hline & Wise RAM - July 2012 & Grundy RAM - October 2012 \\
\hline $\begin{array}{l}\text { Clients seen in the medical } \\
\text { clinic }\end{array}$ & 2,619 & 143 \\
\hline Clients seen by an educator & 297 & 60 \\
\hline $\begin{array}{l}\text { Clients with diabetes seen } \\
\text { by an educator }\end{array}$ & 115 & 36 \\
\hline MyPlate instruction & 107 & 37 \\
\hline $\begin{array}{l}\text { Clients who filled out the } \\
\text { MyPlate survey }\end{array}$ & 68 & 19 \\
\hline $\begin{array}{l}\text { Clients willing to have } \\
\text { follow-up MyPlate survey }\end{array}$ & 57 & \\
\hline
\end{tabular}


Appendix $\mathbf{J}$

Figure 1: MyPlate comprehension

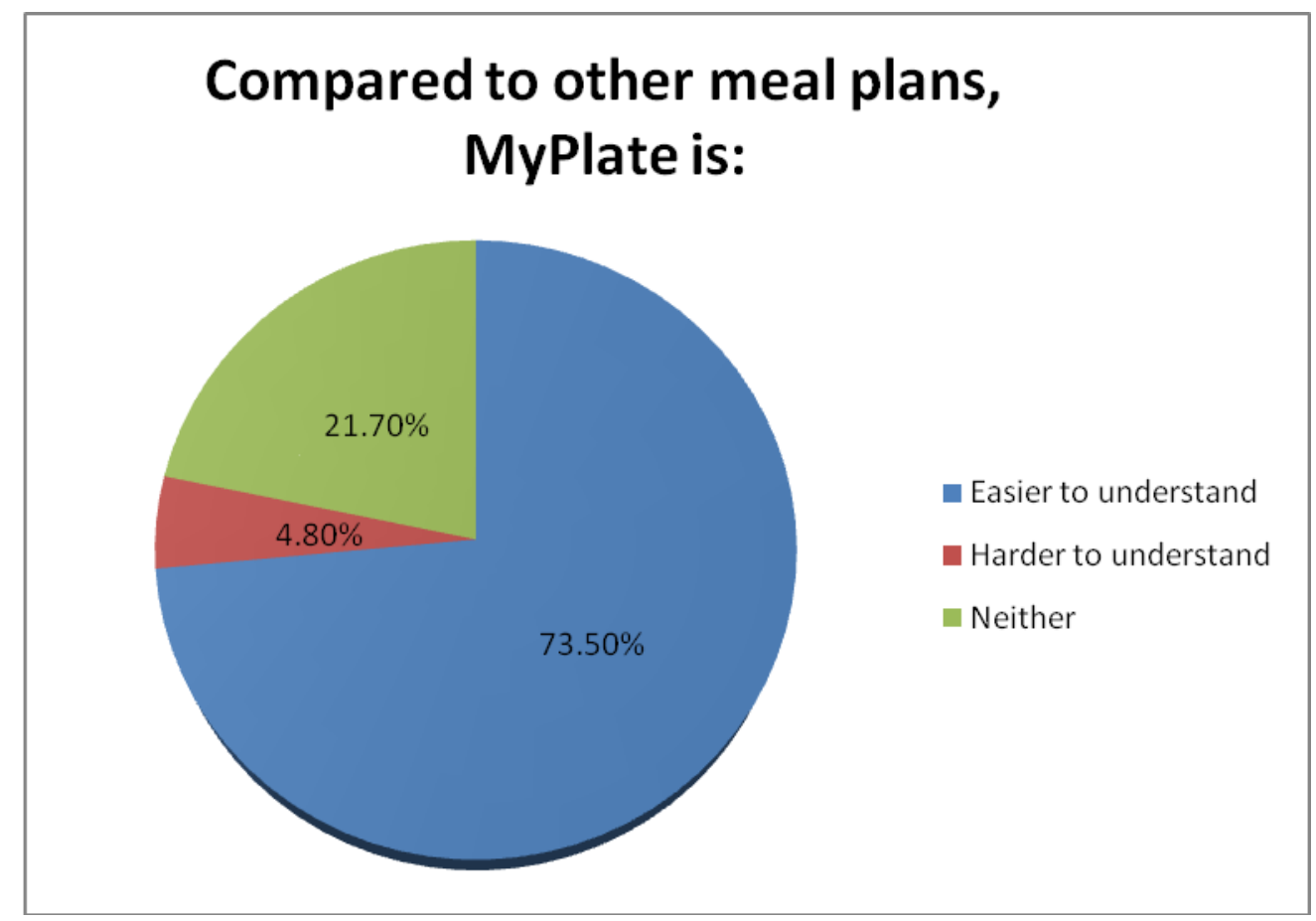

Results based on the survey given to 87 clients instructed on the MyPlate method at the 2012 RAM events. 


\section{Appendix K}

\section{My Plate Evaluation Tool}

Given to participants during the RAM event, immediately after MyPlate Education

RAM/Pt. Ed. \#

1. The My Plate handout was easy to understand: True False

2. I can follow this meal plan when I get home: True False

3. My family will be able to follow this meal plan: True False

4. Compared to other meal plans I have been taught, My Plate was
Easier
Harder About the same

5. When I eat out, this will be,
Easy to follow
Hard to follow Neither

6. This is how I eat

$\begin{array}{lllll}1 & 2 & 3 & 4 & 5\end{array}$

never

Always

7. I will be able to eat like this

$\begin{array}{lllll}1 & 2 & 3 & 4 & 5\end{array}$

never Always

8. If you are not able to eat like this, please check the reason why:

dislike food choices ___ cost ___ big change in eating habits

family will not support this type of food other

9. I can follow this for Breakfast: True False

I can follow this for Lunch: True False

I can follow this for dinner/ supper: True False

10. I want to eat healthier: True False 
Age: Height Weight

Male Female

I have: Heart disease Kidney disease Diabetes Cancer High blood pressure

Can we send you a follow-up survey in 3 months? Yes No

Please return to educator, or Karen Dawn, RN 


\section{Appendix L}

RAM Follow-Up Survey (printed on light blue paper)

Study number: (Pre-filled in prior to mailing to participants)

Date:

1) The MyPlate handout I was given at the 2012 RAM clinic is:
a) On my desk
b) On my refrigerator
c) Put in a place that I do not see every day
d) In my house, but I do not know where it is
e) No longer with me
f) Other

2) I follow the MyPlate food portion size:
a) Always
b) Most of the time
c) Half of the time
d) Some of the time
e) Never

3) If you are not able to follow the MyPlate, please check all the reason(s) that apply. You may check more than one.
a) Dislike food choices
b) Cost
c) Big change in eating habits
d) Family will not support this type of food other 
4) I follow the MyPlate for:
a) Breakfast Yes
No
b) Lunch Yes
No
c) Dinner / Supper Yes
No

5) I want to eat healthier. Yes

No

6) Weight in pounds

7) Has your weight changed since RAM, 2012? Yes

No

Lost ___ number of pounds_ Gained____ number of pounds

8) Have you had any other diet teaching since RAM 2012. Yes No

a) If yes, where

When

What type? Weight Watchers Jenny Craig Nutrisystem

Carb Counting Fat gram counting Atkins

Other

9) I have: Diabetes Pre-Diabetes , High Blood Pressure

Other

Thank you for completing this tool evaluation. Please send this, in the self-addressed and pre-stamped business envelope that came in your original large envelope to the University of Virginia, School of Nursing, P.O. Box 800782, Charlottesville, VA 22908. Please so not write your name on this form. 
Appendix M

Cover Letter, second mailing

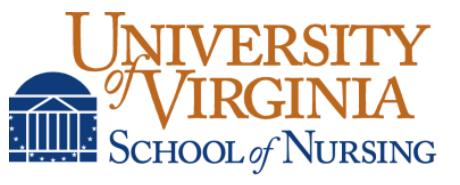

December 6, 2013

P.O. Box 800782

Charlottesville, VA 22908

Dear RAM participant,

I am writing to tell you about a research study that is being done through the University of Virginia. The purpose of this study is to learn whether the MyPlate healthy eating method is a good way of helping people understand and practice healthy eating habits in their daily lives.

We are contacting you because you participated in a teaching session at UVa's Remote Area Medical (RAM) Clinic, using MyPlate.

If you agree to participate, this study will involve filling out the attached survey, which will take about 10 minutes of your time. The survey asks questions about your use of the MyPlate method, and your eating habits.

You may want to know how we obtained your name and information to contact you. We obtained your information from your medical records at UVa's Remote Area Medical (RAM) Clinic. Federal regulations allow the UVa Health System to release your information to researchers at UVa, so that we may contact you regarding studies in which you may be interested in participating.

We want to assure you that we will keep your information confidential, as required by federal privacy regulations. By taking and returning this survey, you agree to allow Dr. Snyder and her study team to use and disclose health information about you to conduct this study. Your information will not be shared outside of this study team except to those groups inside and outside of UVa who are responsible for making sure studies are conducted correctly and ethically.

You do not have to be in this study if you do not want to participate.

Your decision to be in any study is totally voluntary.

Your care at UVa will not be altered by your decision to participate or not participate. 
If you decide to participate in this study now, but decide later to stop, you need to know that the information already collected will continue to be used.

If you are willing to participate in this study, please complete the attached questions and mail it back to us using the enclosed self-addressed, stamped envelope. If we receive the envelope by December 31, 2013, you will receive a $\$ 25$ Food City gift card as a thank you for your time.

If you have any questions, please call the numbers below so that a researcher can talk with you about the study and answer your questions.

If you have any concerns about your rights as a participant in this study you may contact the University of Virginia Institutional Review Board (IRB-HSR\# 17004) by telephone (434-924-2620).

If you have already returned the survey, thank you very much; your gift card is on the way.

Sincerely,

\section{Audrey Snyder}

Audrey Snyder, PhD

Principal Investigator

IRB/HSR \# 17004

Contact information:

Karen Dawn, RN, DNP (c)

karendawn@virginia.edu

Phone: 703-868-3296

Audrey Snyder, RN, PhD

aew3e@virginia.edu

Phone: 434-924-0094 


\section{Appendix N \\ IRB Approval: 10/8/13 and 12/4/13}

UVA IRB OnLine

\section{University of Virginia}

Institutional Review Board for Health Sciences Research

Protection of Human Subjects Approval

Assurance Identification/Certification/Declaration

(Common Federal Rule)

\begin{tabular}{|l|l|l|}
\hline \multicolumn{2}{|c|}{ HSR \# 17004} \\
\hline $\begin{array}{l}\text { Event: } \\
\text { Approval New Protocol - } \\
\text { Expedited }\end{array}$ & $\begin{array}{l}\text { Type: } \\
\text { Protocol }\end{array}$ & $\begin{array}{l}\text { Sponsor(s): } \\
\text { Sponsor Protocol \#: }\end{array}$ \\
\hline & Principal Investigator: Audrey Snyder, PhD \\
\hline
\end{tabular}

Title: Evaluating the MyPlate method to improve patient knowledge and behavior in a rural population at risk of type 2 diabetes

Assurance: Federal Wide Aaserance (FWA)y: 00006183

Certification of IIB Review: The IFB-HSR abides by 2ICFHSO 21CFR56, 46CFR46, 45CFR160, 48CFH164, 32CFR219 and ICH gaidelines. This activity has been reviewed and approved by the IRB in accordance with these renulations.

Approval Date: $10 / 08 / 13$

Protocol Expiration Date: 10/07/14

Approved to Enroll 73 subjects.

HSR Protocol Version Date: 09/25/13

Current Status: Open to enrollment

Consent Version Dates:

Committee Members (did not vote):

Comments: The purpose of this stidy is to evaluate the effectiveness of the MyPlate method as a dietary zuide for Remote Area Medical elinic patients in Southwest Virginia. The study will involve a questionnare maled to subiects which includes evaluation of the tool and basic clinical information. Results of the survey

will be combined with information obtained from the subjects' medical record.

There is no outside sponsor for this study.

No additionat committee approvals are required.

Vulnerable populations being recruited include potential subjects who afe elderly.

$\mathrm{N}=73$, Ages greater than or equal to 18 years:

The following items were reviewed with this approval: questionnaire to be mailed to potential subjects. Compensation vin nlternative route and tax infarmation will not be collected.

Approved with this protocol is a recruitment letter.

REGULATORY INFORMATION:

The IRB determined this protocol met the criteria of minimal risk:

Protocol Expedited by Category \#5: Research involving materials (data, documents, records or specimens\} that have been collected solely for non-rescarch purposes (such as medical treatment and/or diagnosis)

Protocol Expedited by Category \#7: Research on individual or group characteristics or behavior (including, but not limited to, research on perception, cognition, motivation, identity, language, communication, cultural beliefs or practices and social behavior) or research employing survey, interview, oral history, focus group. program evaluation, human factors evaluation, or quality assurance methodologies.

This protocol has been granted a Waiver of Consent to identify potential subjects via 45CFR46.116.

http://www.irb,virginia.edu/index.cfm?fuseAction=hsr_HTMLReports.reportProtocolAssu... 10/8/2013 
This protocol has been granted is Waiver of Consent via 45 CFR 46.116 to contact potential subjects by direct contact by a person who is their health care provider. Direct contact may include phone, letter. direct email or potential subject approached at UVa by a person is their health care provider. Phone. letter or emails will be approved by the IRB-HSR prior to use.

This protocol has been granted in waiver of documentation of consent under 45CFR46.117(c), and an alteration of HIPAA Authorization under 45CFR164.512(ik2) to obtain an oral HiPAA nuthorization for the study. The IRB determined that obtaining written HIPAA authorization would be impracticable because survey will be conducted by mail.

The following HIPAA identifiers will be collected: Name, All geographic subdivisions smaller than a state, all elements of dates directly related to an individual. Medical Record number, For subjects over the age of 89 , their date of birth and age will not be recorded. All will be recorded as greater than 89 years of age,

No identifiable health information will be taken or shared outside of the UV as HIPAA covered entity.

PLEASE REMEMBER:

- Ir an outside sponsor is providing funding or supplies, you must contact the SOM Grants and Contracte Office/ OSP regarding the need for a contract and letter of indemnification. If it is determined that either of these documents is required, participants cannot be enrolled until these documents are complete.

- You must notify the IRB of any new persounel working on the protocol PRIOR to them beginning work.

* You must obtain IRB approval prior to implementing any changes to the approved protocol or consen

form except in an emergency, if necessary to safeguard the well-being of currently enrolled subjects.

- If you are obtaining consent from subjects, prisoners are not allowed to be enrolled in this study. If one of your subjects becomes a prisoner after they are enrolled in the protocol you must notify the IRB immediately.

* You must notify the IRB-HSR office within 30 days of the closure of this study

- Continuation of this study past the expiration date requires re-approval by the IRB-HSR

Conturuation of this study past

The official simming below certifies that the information provided above is correct and that, as required, future reviews will be performed and certification will be provided.

Name: Lynn R, Noland, RN PhD $\quad$ Name and Address of Institution:

Title: Vice Chair, Institutional Review Board

for Health Sciences Research

Phone: 434-924-9634 Fax: 434-924-2932

institutional Review Board for Health

Sciences Research

$\mathrm{PO}$ Bax 800483

\begin{tabular}{l|l} 
Charlottesville, $\sqrt{\mathrm{A}} 22908$ \\
\hline
\end{tabular}

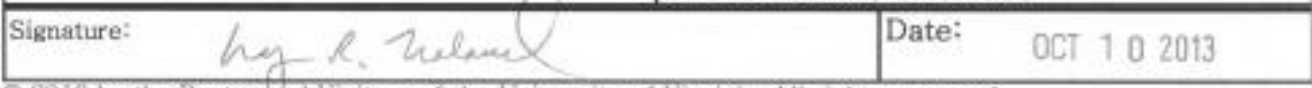

9 2013 by the Rector wind Visitors of the University of Virginia. All rights reserved. 


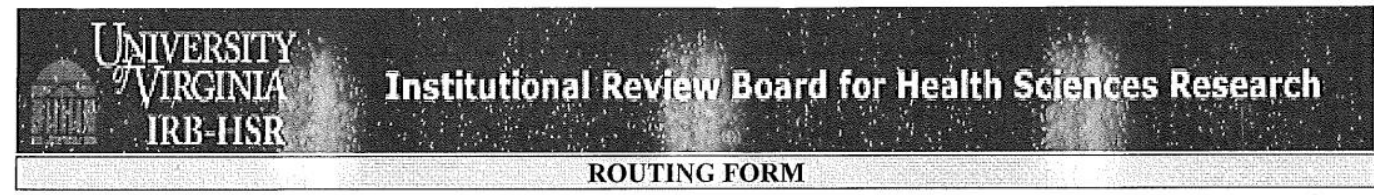

IRB-HSR\#: 17004
Note: The IRB-HSR cannot process this request without one of these numbers.

Complete this form and attach it to the top of ALL submissions to the IRB-HSR Office.

Which IRB staff member did you work with on the pre-review of this submission? Margaret Ball

If another IRB is the IRB of record for this protocol, check an IRB below:

$\square$ NCI CIRB (NCI Cooperative Group protocols)

NeuroNEXT CIRB (NeuroNEXT protocols)

Fred Hutchinson CIRB (CITN protocols)

Western IRB-(NCI Cooperative Group)

Other: Name

Type of Submission:

New Grant

New Protocol-Exempt/ Coded/ Not Engaged

New Protocol Expedited

New Protocol- Full Board

Follow up to Requests from Full Board Review of: $\square$ New Protocol $\square$ Modification

Modification - Attach Appropriate Modification Forms : $\square$ Personnel Change

Adverse Event Report

Advertisement

Protocol Violation/Enrollment Exception

Status Report

Study Closure

Other, Specify:

If multiple documents may submit with Ancillary Document Submission Form

IND/IDE Annual Report **

Revised Investigator Brochure**

**May be submitted as PDF document on Readable Disc (CD) Complete Ancillary Document Submission Form and attach to this

form. The following information must be written on the CD: IRB-HSR \#, Document Name, Document Version Date.

(Check one item below)

No changes required to protocol or consent

Changes required to protocol or consent (attach Modification Request Form and revised protocol and or consent)

\section{CONTACT INFORMATION}

Provide contact information for a representative who can answer any questions the IRB might have concerning this submission:

Approvals/receipt of acknowledgments will also be sent to the person listed below.

Contact Name: Karen Dawn

Phone Number: $703-868-3296$

E-Mail Address: krd6jy@virginia.edu

Messenger Mail Box \#: $\underline{800782}$

Select how you would like the IRB to send the approval/receipt of acknowledgment to you.

$\bigotimes$ Return reply in messenger mail

I will pick up reply at Davis 5- Room 5293 Pick up Box

I will pick up reply from Morton Drive

Replies not picked up within 5 week days will be sent to the contact via Messenger Mail.

The IRB-HSR is unable to send approvals via regular mail unless the individual is not physically located at UVa.

Comments:

Website: http://www.virginia.edu/vpr/irb/hsr/index.html

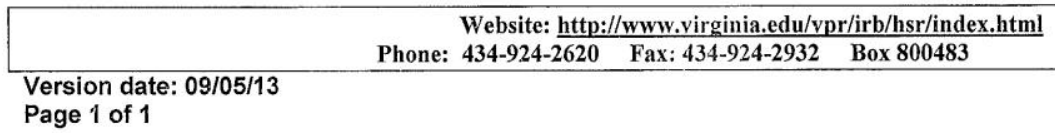




\section{University of Virginia \\ Institutional Review Board for Health Sciences Research}

Protection of Human Subjects Approval

Assurance Identification/Certification/Declaration

(Common Federal Rule)

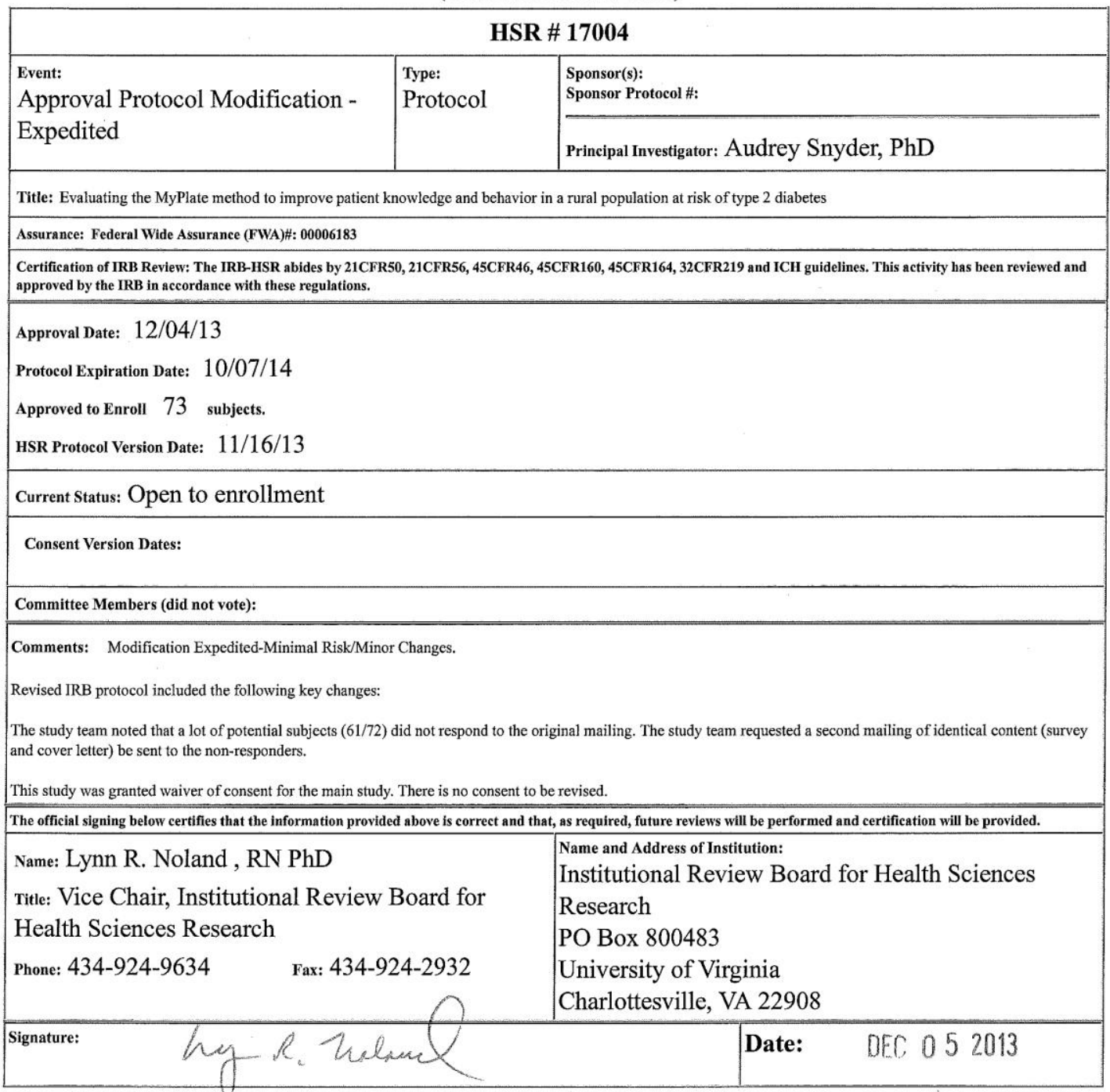

(C) 2013 by the Rector and Visitors of the University of Virginia. All rights reserved. 
Appendix O

Table 3: Baseline and Follow-up Participant Characteristics

\begin{tabular}{|c|c|c|c|c|}
\hline \multirow[t]{2}{*}{ Variables } & \multicolumn{2}{|r|}{ Baseline } & \multicolumn{2}{|r|}{ Follow-up } \\
\hline & $\begin{array}{l}\text { Total } \\
\text { Number }\end{array}$ & $\begin{array}{l}\text { Frequency (\%) OR } \\
\text { Mean (SD) }\end{array}$ & $\begin{array}{l}\text { Total } \\
\text { Number }\end{array}$ & $\begin{array}{l}\text { Frequency (\%) OR } \\
\text { Mean (SD) }\end{array}$ \\
\hline Gender & 87 & & 33 & \\
\hline - Male & & $29(33.3 \%)$ & & $9(27.3 \%)$ \\
\hline - Female & & $58(66.7 \%)$ & & $24(72.7 \%)$ \\
\hline Age & 84 & $\begin{array}{l}\text { Mean: } 46.05(12.87) \\
\text { Range: } 19-78\end{array}$ & 33 & $\begin{array}{l}\text { Mean: } 46.97(10.06) \\
\text { Range: } 22-63\end{array}$ \\
\hline BMI & 84 & $\begin{array}{l}\text { Mean: } 32.34(8.25) \\
\text { Range: } 17.01-55.78\end{array}$ & 31 & $\begin{array}{c}\text { Mean: } 31.41(7.61) \\
\text { Range: } 21.92-56.83 \\
\end{array}$ \\
\hline - $\quad$ Normal $(<25)$ & & $18(21.4 \%)$ & & $8(25.8 \%)$ \\
\hline - Overweight (25-29.99) & & $19(22.6 \%)$ & & $6(19.4 \%)$ \\
\hline - Obese $(>=30)$ & & $47(56.0 \%)$ & & $17(54.8 \%)$ \\
\hline Agree to Follow-up & 87 & & & \\
\hline - No & & $15(17.2 \%)$ & & \\
\hline - Yes & & $72(82.8 \%)$ & & \\
\hline MyPlate is easy to comprehend: & 87 & & & \\
\hline - Yes & & $86(98.9 \%)$ & & \\
\hline - Other & & $1(1.1 \%)$ & & \\
\hline My Family will follow MyPlate: & 82 & & & \\
\hline - True & & $69(84.1 \%)$ & & \\
\hline - False & & $8(9.8 \%)$ & & \\
\hline - Other & & $5(6.1 \%)$ & & \\
\hline I can follow MyPlate at home: & 86 & & & \\
\hline - True & & $83(96.5 \%)$ & & \\
\hline - False & & $2(2.3 \%)$ & & \\
\hline - Other & & $1(1.2 \%)$ & & \\
\hline $\begin{array}{l}\text { Compared to other meal plans, } \\
\text { MyPlate is: }\end{array}$ & 83 & & & \\
\hline Easier to understand & & $61(73.5 \%)$ & & \\
\hline - Harder to understand & & $4(4.8 \%)$ & & \\
\hline - $\quad$ Neither & & $18(21.7 \%)$ & & \\
\hline I can follow MyPlate when I eat out: & 86 & & & \\
\hline - True & & $55(64.0 \%)$ & & \\
\hline - False & & $21(24.4 \%)$ & & \\
\hline - Neither & & $10(11.6 \%)$ & & \\
\hline I follow my Plate portion size now: & 85 & & 31 & \\
\hline - Always & & $7(8.2 \%)$ & & $3(9.7 \%)$ \\
\hline - Most of the time & & $15(17.6 \%)$ & & $9(29.0 \%)$ \\
\hline - Half of the time & & $49(57.6 \%)$ & & $6(19.4 \%)$ \\
\hline - Some of the time & & $11(12.9 \%)$ & & $9(29.0 \%)$ \\
\hline - Never & & $3(3.5 \%)$ & & $4(12.9 \%)$ \\
\hline I will be able to eat like MyPlate: & 85 & & & \\
\hline • Never & & $0(0 \%)$ & & \\
\hline
\end{tabular}




\begin{tabular}{|c|c|c|c|c|}
\hline Variables & \multicolumn{2}{|c|}{ Baseline } & \multicolumn{2}{|c|}{ Follow-up } \\
\hline - 2 & & $4(4.7 \%)$ & & \\
\hline - 3 & & $30(35.3 \%)$ & & \\
\hline - 4 & & $31(36.5 \%)$ & & \\
\hline - $\quad$ Always & & $20(23.5 \%)$ & & \\
\hline Barriers to following MyPlate: & & & & \\
\hline - $\quad$ Dislike food & & $10(11.5 \%)$ & & \\
\hline - Cost is prohibitive & & $17(19.5 \%)$ & & $15(45.5 \%)$ \\
\hline - $\quad$ Big change in eating habits & & $9(10.3 \%)$ & & $10(30.3 \%)$ \\
\hline - Other barriers & & & & $9(27.3 \%)$ \\
\hline - No family support & & $4(4.6 \%)$ & & \\
\hline I can follow MyPlate for Breakfast & 84 & & 27 & \\
\hline - True & & $74(85.1 \%)$ & & $11(40.7 \%)$ \\
\hline - $\quad$ False & & $6(6.9 \%)$ & & $16(59.3 \%)$ \\
\hline$\bullet \quad$ Other & & $4(4.8 \%)$ & & \\
\hline I can follow MyPlate for Lunch & 83 & & 29 & \\
\hline - True & & $74(89.2 \%)$ & & $15(51.7 \%)$ \\
\hline - $\quad$ False & & $4(4.8 \%)$ & & $14(48.3 \%)$ \\
\hline - Other & & $5(6.0 \%)$ & & \\
\hline I can follow MyPlate for Dinner & 85 & & 28 & \\
\hline$\bullet \quad$ True & & $78(97.8 \%)$ & & $16(57.1 \%)$ \\
\hline - $\quad$ False & & $3(3.5 \%)$ & & $12(42.9 \%)$ \\
\hline - Other & & $4(4.7 \%)$ & & \\
\hline I want to eat Healthier & 84 & & 30 & \\
\hline$\bullet \quad$ True & & $81(96.4 \%)$ & & $28(93.3 \%)$ \\
\hline - $\quad$ False & & $2(2.4 \%)$ & & $2(6.7 \%)$ \\
\hline • Other & & $1(1.2 \%)$ & & \\
\hline I have heart disease & 87 & & & \\
\hline$\bullet \quad$ No & & $82(94.3 \%)$ & & \\
\hline - Yes & & $5(5.7 \%)$ & & \\
\hline I have diabetes & 87 & & 33 & \\
\hline - No & & $54(62.1 \%)$ & & \\
\hline - $\quad$ Yes & & $33(37.9 \%)$ & & $16(48.5 \%)$ \\
\hline I have hypertension & 87 & & 33 & \\
\hline - $\mathrm{No}$ & & $42(48.3 \%)$ & & \\
\hline - Yes & & $45(51.7 \%)$ & & $15(45.5 \%)$ \\
\hline Stated weight change & & & 32 & \\
\hline - Yes & & & & $26(81.2 \%)$ \\
\hline$\bullet \quad \mathrm{No}$ & & & & $6(18.8 \%)$ \\
\hline Stated weight lost or gained & & & 22 & \\
\hline - Lost & & & & $18(81.8 \%)$ \\
\hline • $\quad$ Gained & & & & $4(18.2 \%)$ \\
\hline Other diet education since RAM & & & 30 & \\
\hline - No & & & & $27(87.1 \%)$ \\
\hline - Yes & & & & $4(12.9 \%)$ \\
\hline
\end{tabular}




\section{Appendix P}

Figure 2: Ability to Follow MyPlate Portion Size, baseline and follow-up

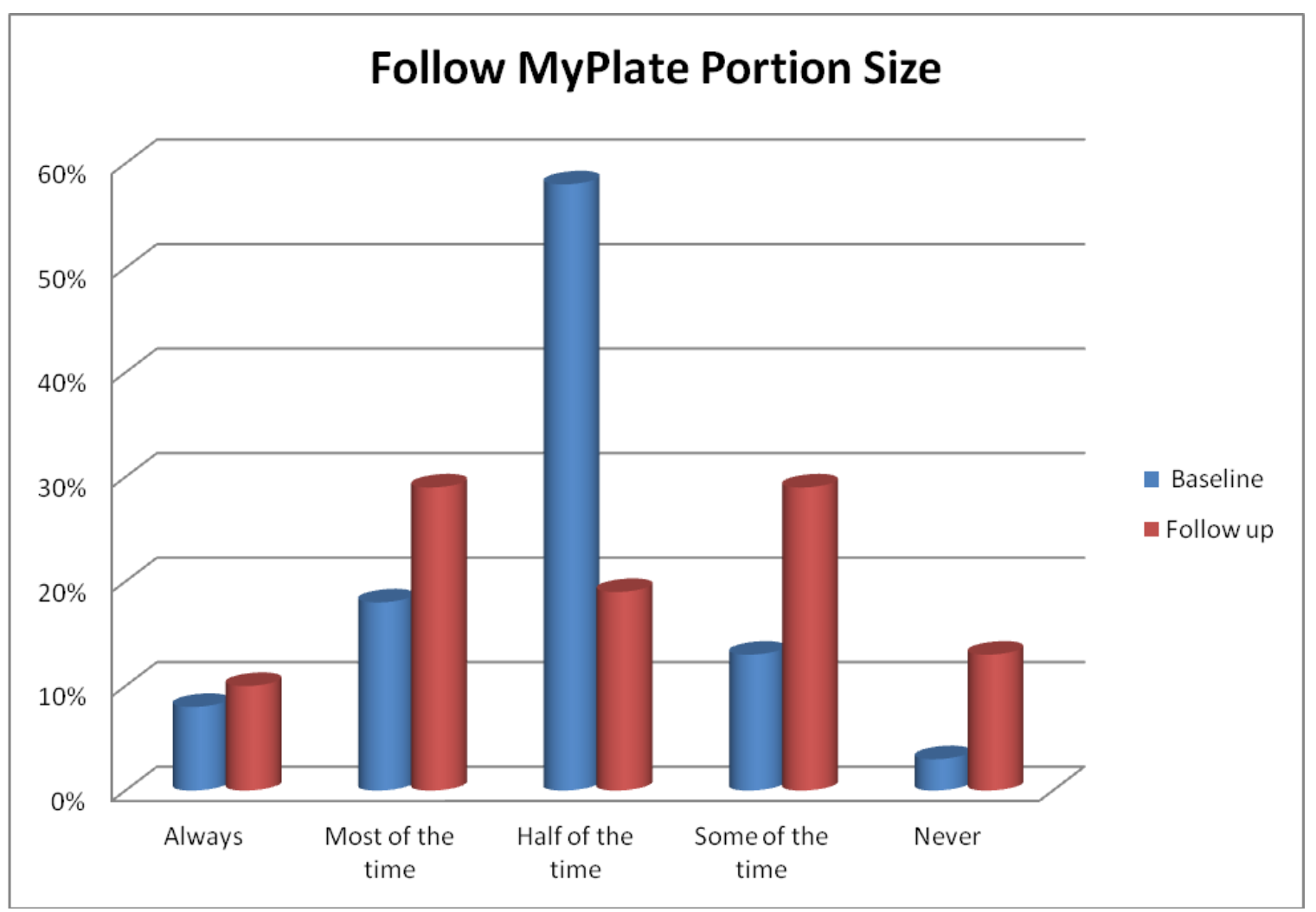

Participants were asked at baseline, "This is how I eat now", with a Likert scale response. At follow-up, the Likert scale was changed to the above label check off. This change in measurement (Likert scale to check list) may account for the large discrepancy. 
Appendix Q

Figure 3: Perceived and Actual Ability to Follow MyPlate by Meal

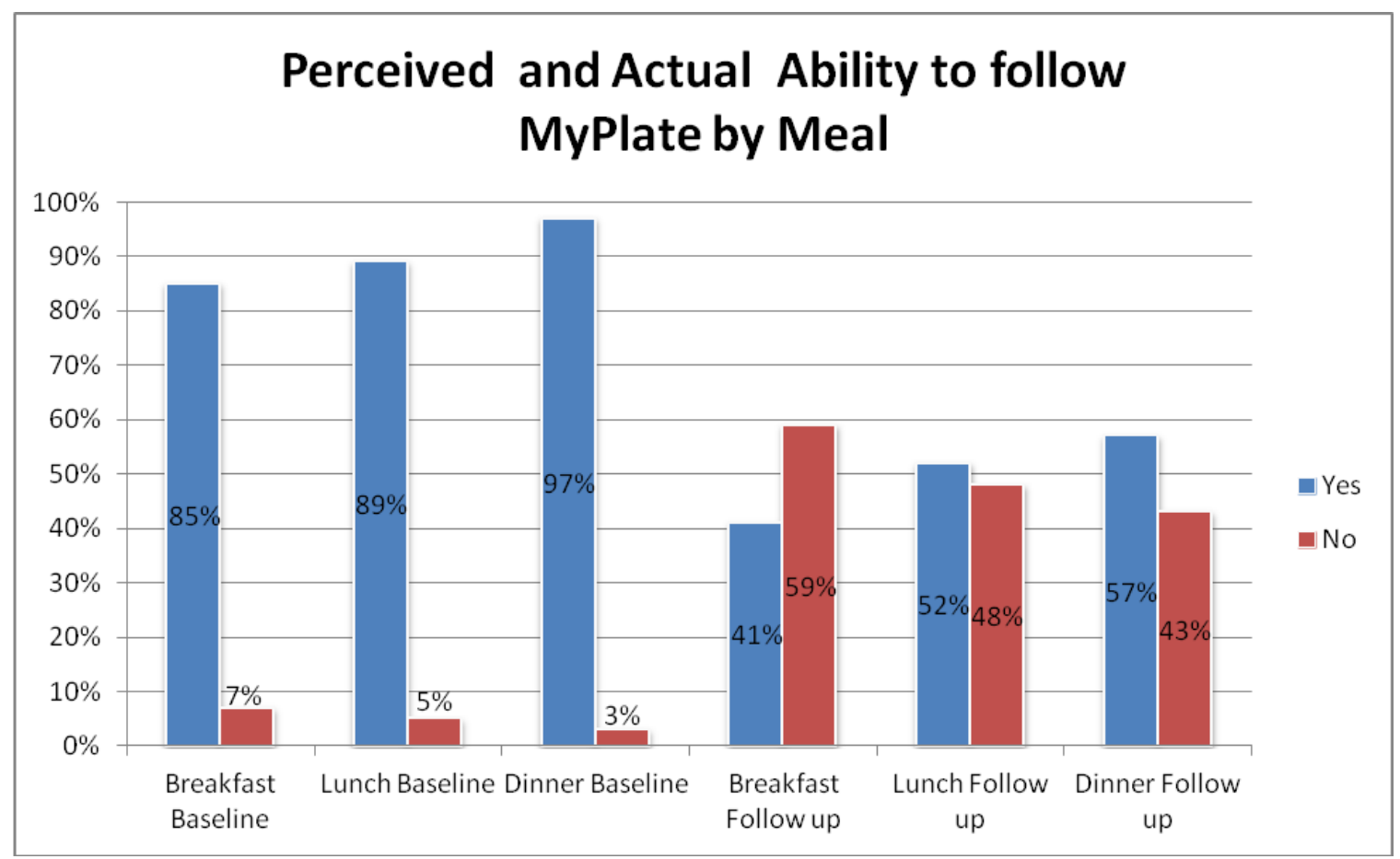

Participants were asked at baseline "I can follow MyPlate for breakfast," "I can follow MyPlate for lunch," and "I can follow MyPlate for dinner/supper." At follow-up, the questions were, "I follow MyPlate for breakfast," "I follow MyPlate for lunch," and "I follow MyPlate for dinner.” Participants perceived they would follow MyPlate more than they actually did. 
Appendix R

Figure 4: Responder Weight Loss in pounds at Baseline and Follow-up

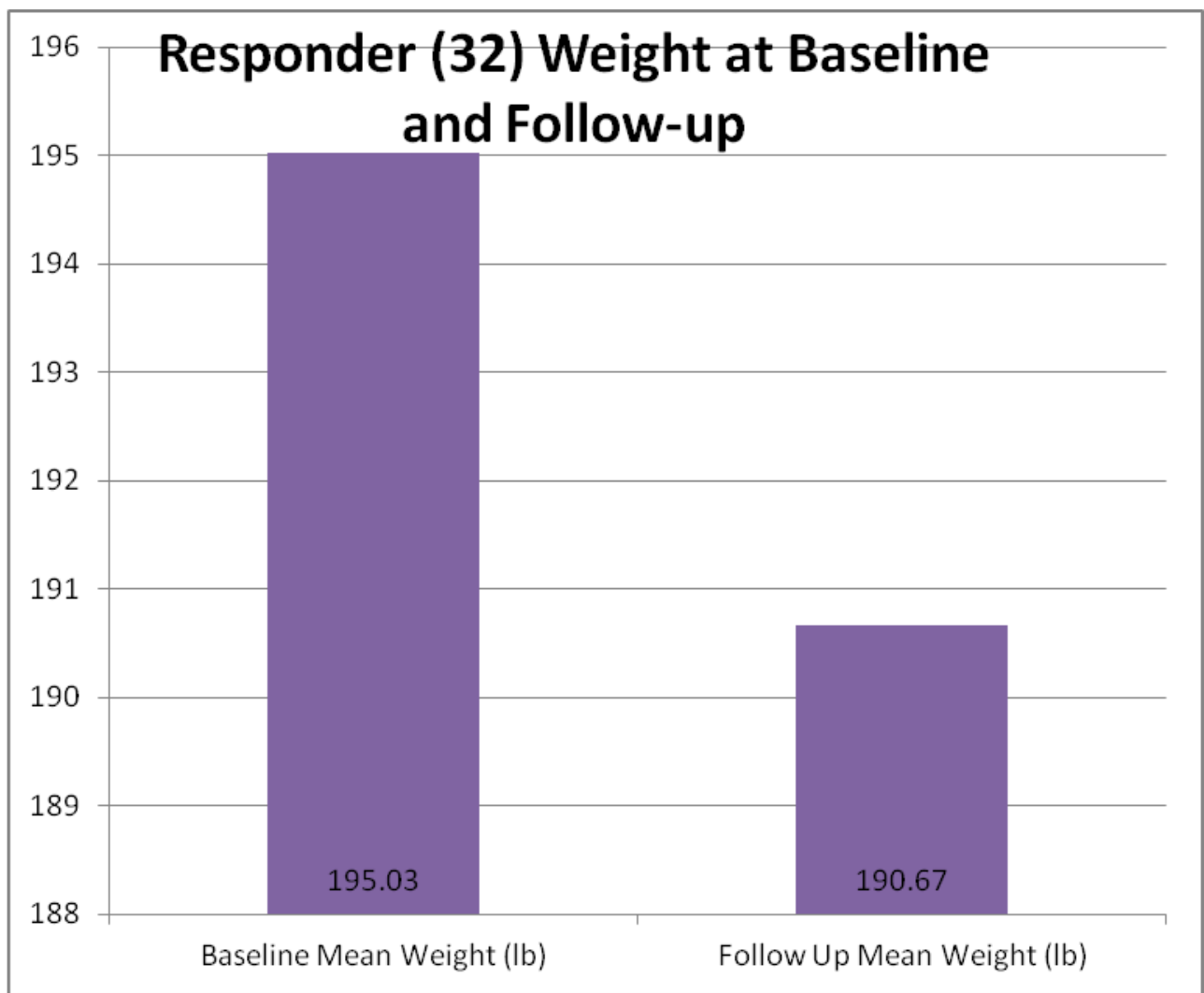

Mean baseline weight was 195.03 pounds $(\mathrm{SD}=42.47)$. The mean weight for follow-up was 190.67 pounds ( $\mathrm{SD}=45.62)$. The difference (4.36 pounds weight loss) is not statistically significant, $\mathrm{t}=1.45, \mathrm{df}=32, \mathrm{p}=0.158$. 
Appendix S

Figure 5: Weight Change in Responders by Category

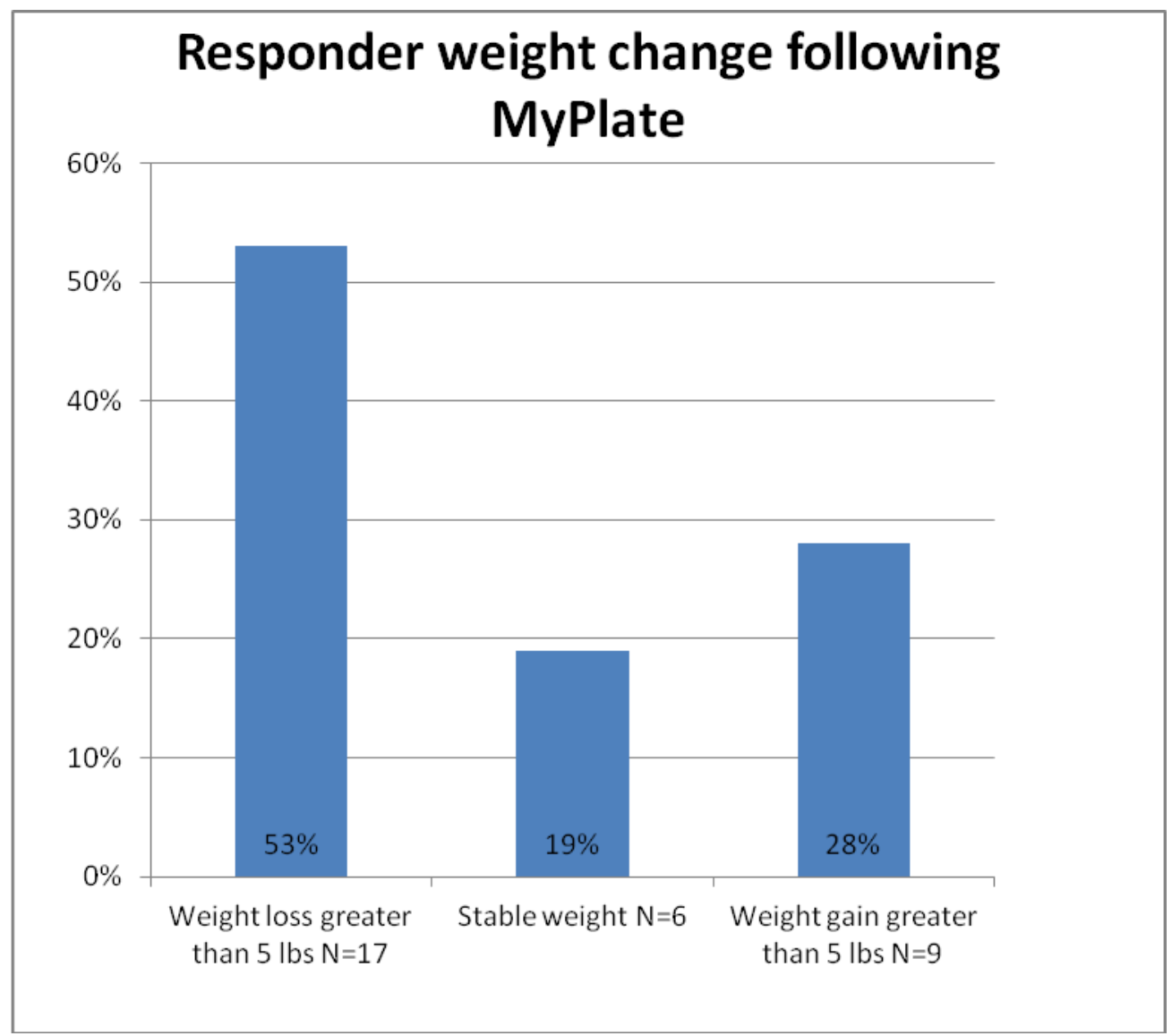


Appendix T

Table 4: Gender Difference and Weight Change

\begin{tabular}{|c|c|c|c|c|}
\hline & $\begin{array}{c}\text { Lost 5 pounds } \\
\text { or more } \\
\mathrm{N}(\%)\end{array}$ & $\begin{array}{c}\text { Stable Wt, } \\
\text { within 5 } \\
\text { pounds change } \\
\mathrm{N}(\%)\end{array}$ & $\begin{array}{c}\text { Gained 5 } \\
\text { pounds or } \\
\text { more } \\
\mathrm{N}(\%)\end{array}$ & total \\
\hline Males & $4(44 \%)$ & 0 & $5(56 \%)$ & 9 \\
\hline Females & $13(57 \%)$ & $6(26 \%)$ & $4(17 \%)$ & 23 \\
\hline $\begin{array}{c}\text { Participants in } \\
\text { each weight } \\
\text { category }\end{array}$ & $17(53 \%)$ & $6(19 \%)$ & $9(28 \%)$ & 32 \\
\hline
\end{tabular}

Fisher's exact $=5.24, \mathrm{p}=0.074$. 
Appendix U

Figure 6: MyPlate Tool Visibility and Mean Pound Weight Loss

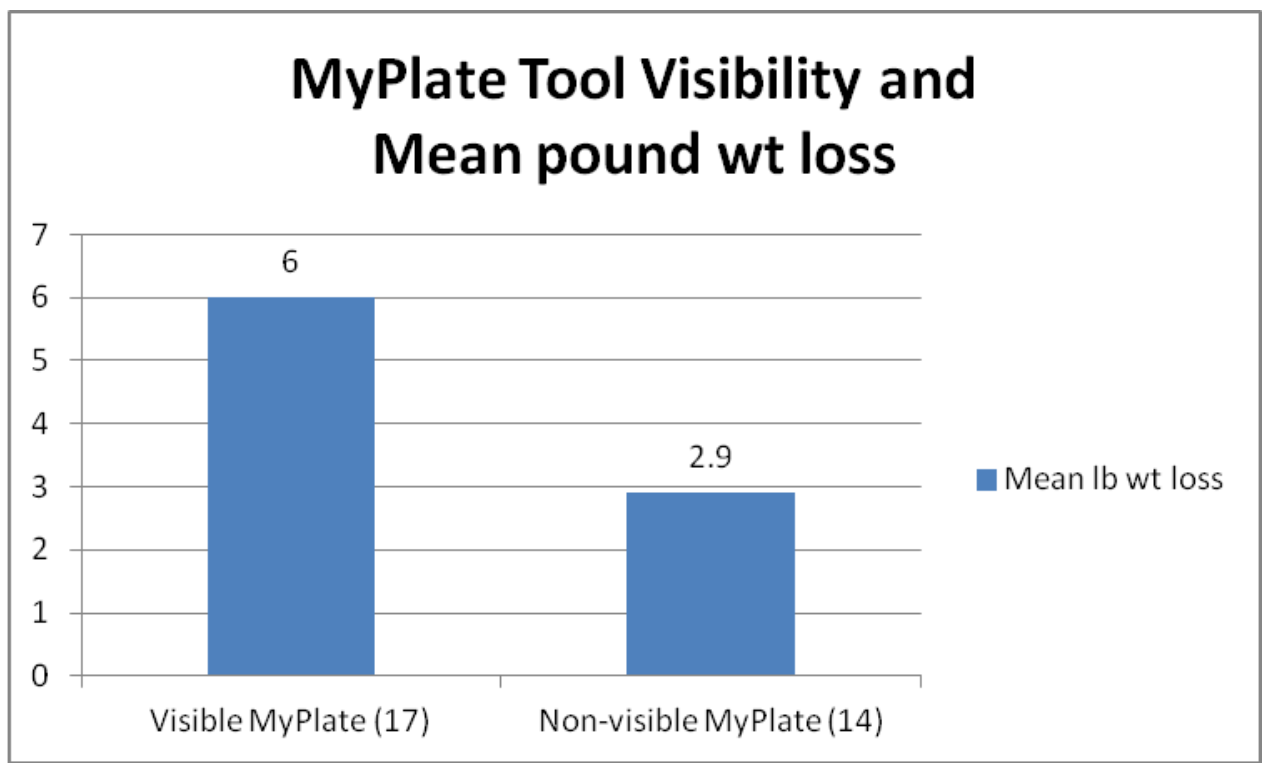

Weight loss in pounds, $\mathrm{t}=0.49, \mathrm{df}=29, \mathrm{p}=0.63$ 
Appendix V

Figure 7- MyPlate Visibility and Weight Change
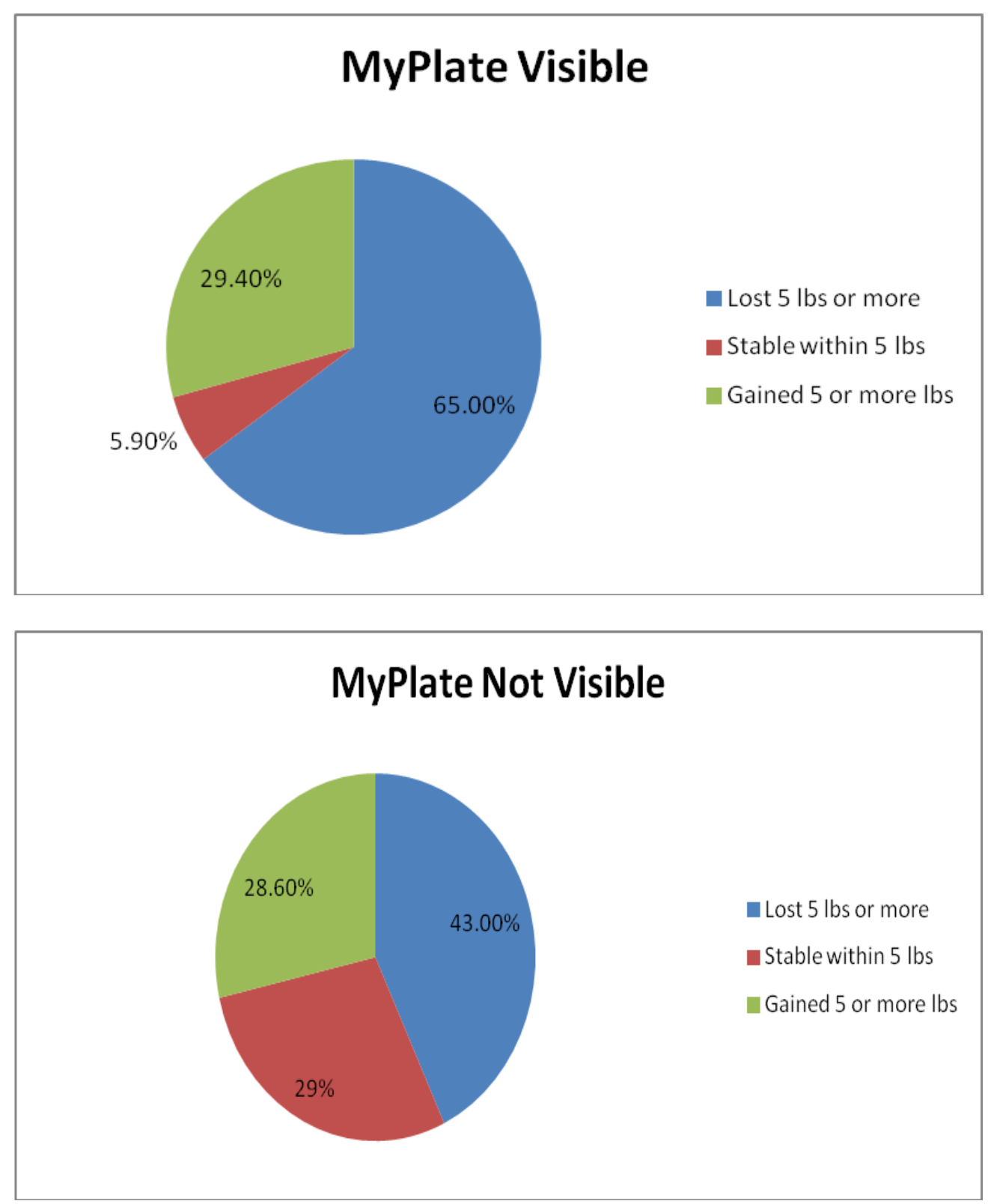

Chi-square $=7.27, \mathrm{p}=0.104$

Cramers' $\mathrm{V}=0.337, \mathrm{p}=0.122$

Although the results were not statistically significant, the Cramers' V indiciates a moderate effect size. 
Appendix W

Author Guidelines

\section{Writing for the American Journal of Nursing: Author Guidelines}

$A J N$ welcomes submissions of evidence-based clinical application papers and descriptions of best clinical practices, original research reports, case studies, narratives, commentaries, and other manuscripts on a variety of clinical and professional topics. The journal also welcomes submissions for its various departments and columns, including artwork and poetry that is relevant to nursing or health care. Guidelines on writing for specific departments - Art of Nursing, Viewpoint, Policy and Politics, and Reflectionsare available below and at http://AJN.edmgr.com.

$A J N$ 's mission is to promote excellence in nursing and health care through the dissemination of evidence-based, peer-reviewed clinical information and original research, discussion of relevant and controversial professional issues, adherence to the standards of journalistic integrity and excellence, and promotion of nursing perspectives to the health care community and the public.

$A J N$ is a peer-reviewed journal that follows publishing standards set by the International Committee of Medical Journal Editors (ICMJE; www.icmje.org), the World Association of Medical Editors (WAME; www.wame.org), and the Committee on Publication Ethics (COPE; http://publicationethics.org/). For original research, it also uses the Consolidated Standards of Reporting Trials (CONSORT; www.consort-statement.org) for randomized studies; the Transparent Reporting of Evaluations with Nonrandomized Designs (TREND; http://www.cdc.gov.proxy.its.virginia.edu/trendstatement/) for descriptive and epidemiologic studies, and the Standards for Quality Improvement Reporting (SQUIRE; www.squire-statement.org) for quality improvement reports.

Manuscripts are subject to double-blind peer review. Before submitting a paper to AJN, authors need to be aware that all accepted papers undergo intensive clinical and grammatical editing that includes fact-checking, reference checking, determinations of balance and accuracy, and overall editing to enhance the readability and accessibility of the paper. Submission of a manuscript implies the authors' agreement to work on the manuscript with the editorial staff —on a continuing basis—during production, and this agreement is confirmed before papers are accepted. Poems and artwork are not edited. For more information on $A J N$ 's editing process, go to http://edmgr.ovid.com.proxy.its.virginia.edu/ajn/accounts/expect.doc.

PLEASE NOTE CAREFULLY: The journal will only review manuscripts formatted according to the style of the American Psychological Association (APA; 
www.apastyle.org), as detailed below. Do not submit papers with references formatted in Endnotes.

\section{QUERY LETTERS}

We encourage authors to familiarize themselves with the journal in print or online at www.AJNonline.com. Query letters should include a paragraph describing the proposed manuscript, its projected length, how the topic will be treated, an abstract and outline, a short biographical sketch that includes the author's qualifications for writing on the topic, and the author's contact information (e-mail and street addresses and daytime and evening telephone numbers). Do NOT send the manuscript. Query letters should be sent to alison.bulman@wolterskluwer.com. We do consider completed manuscripts submitted through http://ajn.edmgr.com without querying first, however queries allow us to determine whether the topic is an editorial priority at this time and if the manuscript should be submitted through Editorial Manager for peer review.

Authors may send query letters to an unlimited number of journals simultaneously. However, it is not appropriate for authors to submit a manuscript to more than one journal at a time. We do not consider manuscripts that are being reviewed by another publication or previously published manuscripts. Authors who violate this standard of biomedical publishing will not be welcome to submit other manuscripts to the journal.

\section{SUBMISSION}

Authors must submit all manuscripts online at http://ajn.edmgr.com. Log on to register and submit a manuscript. For questions about submitting a manuscript, contact Alison Bulman, Associate Editor (alison.bulman@wolterskluwer.com) at (646) 674-6602.

For details on acceptable program formats for documents, look on the main page under the Files and Resources heading and click on the System Requirements link. To accommodate blinded peer review, authors enter their names and the names of their institutions directly into the Web site, as instructed, and that information will remain linked to their manuscripts. Names and affiliations should appear only in the cover letter and NOT anywhere on the manuscript Word doc itself. This is a secure site; $A J N$ editors control access to all submissions.

The cover letter. Authors should submit a cover letter with each manuscript. The cover letter should include the following:

- a description of any other submissions or previous publications that might be considered redundant or that duplicates any part of the submitted manuscript. Copies of such publications should be faxed to (212) 886-1206 or mailed to the $A J N$ editorial office at 333 Seventh Avenue, 20th Floor, New York, NY 10001; alternatively, a link to an online version of the article can be included in the cover letter.

- a description of the steps the author(s) took to ensure the anonymity of any people discussed in the manuscript (in clinical scenarios or case studies, for instance; see Anonymity and Patient Confidentiality, below). 
- A statement about whether permission has been obtained for reprinting or adapting any tables, charts, illustrations, or other parts of the manuscript that have been previously published.

Conflicts of interest and authorship. $A J N$ adheres to journalistic standards that require transparency of real and potential conflicts of interests that authors and editors may have. All contributing authors must fill out the AJN Author Agreement form (available on the Editorial manager homepage), and e-mail it to their lead/corresponding author to submit with their manuscript during the step-by-step submission process through the system. Manuscripts will not be reviewed without submission of this document, which includes the following:

Financial Disclosure. This is an acknowledgment of all funding sources that supported the submitted work, as well as any commercial association that could pose a conflict of interest or create a bias. Such associations include, but are not limited to, payments for consulting, honoraria for speaking or writing on the topic discussed in the manuscript, patent licensing arrangements, or payments for conducting or publicizing a study or other work or product mentioned in the article. Authors must provide a detailed explanation of any writing, editing, or review of the manuscript by any organization or party that has a financial or other interest in the manuscript.

Authorship. Any individuals who participated in writing the manuscript but are not listed as authors in the cover letter should all complete the form, including any individuals who were paid directly for writing the manuscript and the source of the funding. (Anyone who contributed to the project but not as an author of the manuscript may be named in the acknowledgments, with a description of the person's role in the project. The journal must receive the acknowledged person's written permission to be acknowledged in the published article.)

Copyright Transfer. The copyright transfer is activated once the article is published; however, the journal requires that the authors indicate their intent to transfer copyright to the journal by signing this form when the manuscript is submitted.

Photographs and art. Authors who submit original artwork or photographs should post clear versions with the manuscript. High-resolution images are required (see Tables, Figures, Illustrations, and Photos below for the specifications required). Authors who submit artwork and photos by mail should include a self-addressed envelope suitable for returning the materials safely. Authors may also contact Associate Editor Alison Bulman if they have questions or need assistance with the online submission of such materials. She can be reached at alison.bulman@ wolterskluwer.com or (646) 674-6602.

\section{REVIEW PROCESS}

$A J N$ uses double-blind peer review in the consideration of most manuscripts, including many of the different columns. Other columns will receive internal peer reviews in which the authors' identities are known. 
The peer-review process provides authors and the journal editors with critiques and recommendations based upon expert knowledge. It doesn't ensure that manuscripts are accurate, free of plagiarism, readable, or balanced. As noted above, the journal heavily edits accepted papers to ensure the quality and readability of the paper. Additionally, all manuscripts are screened for plagiarism.

Initial reviews usually take four to six weeks from the date of submission, although expedited reviews can be provided for original research and other manuscripts with timesensitive data. Authors who believe their manuscripts are time sensitive should contact Editor-in-Chief Shawn Kennedy, MA, RN, at shawn.kennedy@ wolterskluwer.com before submitting the manuscript.

After evaluation of the initial peer reviews, an $A J N$ editor will inform the author of the manuscript's status or decision by e-mail. Please note that most of the manuscripts published in $A J N$ are revised before acceptance. Authors can log on to http://ajn.edmgr.com at any time to check the status of their submissions. Authors who do not receive notice of a decision within eight weeks of submission may contact the editorial offices for an update on the manuscript's status.

A decision of "accept with revision" is not a commitment to accept the paper if the author fails to make the recommended changes. A rejection may be appealed by e-mailing Alison Bulman at alison.bulman@wolterskluwer.com.

General Guidelines. The style of a submission depends on the type of article being written. For example, manuscripts reporting original research should follow the format described below and in more detail in the "Uniform Requirements for Manuscripts Submitted to Biomedical Journals" (www.icmje.org). Pieces submitted to AJN's Art of Nursing, Reflections, or Viewpoint departments should be organized according to criteria specific to these pages as noted below and after careful review of previously published samples found on our Web site www.ajnonline.com. Authors are encouraged to write in active voice, and first and second person are permitted as appropriate.

Presentation. Type the manuscript with margins of $1 \frac{1}{2}$ " on all sides, left justified only. Type must be a minimum of 12-point font size. Double-space throughout and arrange the text in the following sequence: acknowledgments should appear only in the cover letter; abstract with key words for indexing; main text; references; and figures, tables, and illustrations when appropriate. Number the pages consecutively, in the upper righthand corner. Include a short title in the header of each page, just above the page number. We also prefer that you use line numbers.

We encourage authors to use headings and subheadings throughout the manuscript. We also encourage the use of diagrams, tables, charts, illustrations, and photos, all with appropriate permissions from sources and from those persons pictured (see below for details); however, authors should include these as attachments, as opposed to placing them in the body of the text. Use generic drug names instead of proprietary names whenever possible. If it's necessary to use trade names, they should be capitalized and 
inserted within parentheses after the generic name on first mention. Thereafter, the generic name should be used, if possible. Product names should be treated likewise, with the manufacturer's full name and the city in which the product is manufactured in parentheses.

Length. AJN does not have a limit on page length for feature articles; however, papers over 20 pages in length (14,000 words) are discouraged. Do not cut the details of a paper or reduce font size in order to get the manuscript to under 20 pages. We prefer to advise authors on reducing length after their papers are peer reviewed. Columns are shorter, more tightly focused and generally run 1500 - 2500 words. Submissions for Reflections and Viewpoint should be no more than 850 words.

Sourcing. Authors must provide proper sourcing throughout their papers. Primary sources should be used. Textbooks, dictionaries, and encyclopedias (including Wikipedia) are seldom appropriate references. For more specifics on primary versus secondary sources, see $A J N$ 's policy "Primary and Secondary Sources" under "Author Guidelines" at http://AJN.edmgr.com.

All direct quotes - including those from government sources, online publications, and publications that are in the public domain - must be presented as such, with quotation marks and appropriate citations. Treating direct quotes otherwise will be considered plagiarism. In addition, manuscripts that contain plagiarism or have extensive poor paraphrasing may be rejected, even after acceptance. The journal highly recommends that authors review the journal's plagiarism policy at http://AJN.edmgr.com under "Author Guidelines".

References. Authors are responsible for the accuracy and completeness of their references and for correct text citation (see Sourcing, above). For editing purposes, manuscripts with references must be formatted in scientific notation, as defined by the American Psychological Association (APA; www.apastyle.org). Do not submit papers with references formatted in Endnotes.

Anonymity and Patient Confidentiality. We encourage authors to include clinical scenarios or case studies in their manuscripts. It is imperative to protect the anonymity of people described in these scenarios or to obtain the written consent of all such people, even if individuals' names are not used, whenever the identity of such a person could reasonably be determined.

Descriptions of clinical scenarios involving patients need to be identified as real or composites. Case studies or detailed reports of cases used as data must be accompanied by the written permission of the patient and family included in the case report. Authors may not change the details of a case, but cases can be de-identified to ensure the patient's anonymity. The journal will work with authors on de-identifying procedures if the manuscript is accepted; however, most patients and families are eager to have their cases shared for learning purposes as long as their names are not used. For brief patient scenarios, authors may construct composite descriptions that are representative of a 
patient population but not based upon one real case, or one case can be used providing it does not include details that would jeopardize the patient's anonymity. When a composite is created, the author needs to state this in the manuscript; for example, "Jane Smith was a 70 year old woman who sustained a fall at home (this case is a composite based upon the author's experience)." Authors who include real cases of any kind in their manuscripts must complete a "Patient Case Permissions Form" available at http://AJN.edmgr.com under "Author Forms."

Continuing Education Questions. Do not submit test questions with your manuscript. The editors will determine whether your manuscript is suitable to be a continuing education feature in the journal. If it is, the test questions will be developed independently.

\section{TYPES OF SUBMISSIONS}

Clinical Application Papers. AJN welcomes papers that synthesize research and other evidence to describe best clinical nursing practices. We encourage authors to focus the paper on an area in which there is new knowledge or advances in defining best practices. Authors should ensure that their papers describe the evidence for practice, including a description of the literature search, existing research that is relevant, and gaps in the current literature. Authors should not feel compelled to include discussion of all research but should focus instead on the most important, best-designed studies. Papers that include discussion of the findings of a systematic review should include the review's grading of the evidence in parentheses for each practice recommendation, a statement about the organization that conducted the review, and references and Internet links to the original review and the grading system. Evidence tables should be included with systematic reviews.

Clinical papers should clearly define the paper's focus, which should be broad enough to be of interest to $A J N$ 's broad-based audience but sufficiently narrow to present the topic with depth and specificity. Authors should describe in detail how the ideas and information presented can be directly applied in clinical practice. Clinical papers on health problems should include a discussion of the relevant epidemiology; assessments and diagnostics; medical treatments (including pharmacologic therapies) and their advantages, disadvantages, and nursing implications; the physical, psychosocial, and educational issues confronted by patients, families, and nurses caring for them; and sufficient detail on nursing interventions. Essentially, the reader will ask, "What's in this article for me and for my patients? What can I take away from this piece that will improve the care I provide, my patients' lives, or my professional life?" Provide examples and details of recommended assessments or interventions. For example, it is not sufficient to state, "provide emotional support to the patient." Instead, the author should provide sample dialogue or explanations of the behaviors that would illustrate such support.

Quality Improvement Studies. $A J N$ welcomes papers on practice innovations and quality improvement (QI) that include detailed descriptions of the innovation or intervention and outcome data. Authors should refer to the SQUIRE guidelines for a 
description of what should be included in QI papers (see www.SQUIRE-statement.org). Not all areas included in the SQUIRE guidelines must be addressed in detail in a QI manuscript, but the author should ensure that the major themes in the guidelines are adequately addressed.

Original Research. For original research, authors should refer to the guidelines set forth by the ICMJE (www.icmje.org), although references should be submitted in APA format.

All interventional studies should be registered in an approved, publicly accessible clinical trial registry such as www.clinicaltrials.gov (see http://jama.amaassn.org.proxy.its.virginia.edu/misc/authors.dtl for a list of approved research registries). Authors must include the registration number in the cover letter. Authors of interventional research not registered should contact the editor-in-chief for a waiver before submitting the manuscript.

Original research manuscripts should follow reporting guidelines endorsed by the ICMJE; the CONSORT Group (www.CONSORT-statement.org) for randomized clinical trials, including the checklist for authors (which can be found at www.CONSORTstatement.org); and the TREND Group's statement on Transparent Reporting of Evaluations of Nonrandomized Designs (http://www.cdc.gov.proxy.its.virginia.edu/trendstatement/) for studies with nonrandomized.

Reports on quantitative studies should include the following sections:

- A structured abstract that includes a statement of the problem and its significance, the study design, the sampling method, the variables examined, the most important finding(s), and most important conclusion(s)

- Introduction stating the problem and its significance

- Background or literature review

- Statement of the study aims, research questions, and/or hypotheses

- Methods, including study design; a statement showing institutional review board approval and procedures for obtaining informed consent from or for subjects; details of the sampling plan and instruments; and protocols or procedures (please include as an attachment a copy of the protocol if the research is an interventional study)

- Findings or Results, including flow of participants throughout the study using the CONSORT Group guidelines (the flow diagram of the CONSORT Group is available at www.CONSORT-statement.org); descriptive statistics with absolute numbers as well as summary statistics (for example, mean, SD, and percentages, as appropriate); and inferential statistics (for example, $t$ test $=3.41$; $\mathrm{df}=10 ; P=$ 0.002 ) for all relationships tested.

- Discussion, including examination of the findings within the context of other research; limitations of the study; and recommendations for practice, policy, and future research. 
- All data display charts must include actual data points; for example, bar graphs must include the actual end point datum for the bar. (See "Tables, Figures, Illustrations, and Photos" above.)

Reports on qualitative studies should follow the same format as above but with appropriate detail on and referencing of study approach, including design, sampling determinations, methods of data analysis, findings that include sample demographic data and qualitative data, and discussion of the findings within the context of prior research and theory.

Authors of all study reports should also be mindful of the rules on the ethical conduct of research. During the peer-review or editing processes, questions about the accuracy of the reported data may lead to a request by $A J N$ to have the data independently evaluated by a statistician whose selection is mutually agreed upon by the author and editor-in-chief.

Researchers should refrain from soliciting media coverage of the results of their research prior to publication. $A J N$ follows the Ingelfinger Rule, which limits such coverage to journalists' reporting on what researchers present at peer-reviewed conferences and meetings without the researcher elaborating beyond the presentation or giving data or slides to the journalists. For more information on the guidelines for researchers' contact with media prior to publication of their work, go to http://www.icmje.org/\#media. 


\title{
Appendix X
}

Manuscript

\section{Title}

A Quasi-Experimental Study Evaluating the MyPlate Teaching Tool in an Obese Rural Population at Risk for Type 2 Diabetes

\begin{abstract}
The rural population of Appalachia is plagued by enormous health disparities due to extensive poverty, lack of education, and minimal access to health care. Type 2 diabetes and obesity have become regional epidemics. Because there are limited health care providers and many barriers to accessing treatment within Appalachia, the Remote Area Medical (RAM) clinic has been an important initiative in addressing the health care needs of the region. For the 2012 RAM events, nurse educators implemented a nutrition education project, based on Pender's Health Promotion Model, using the MyPlate educational tools targeting individuals who were at risk for developing or who have type 2 diabetes (mean $\mathrm{BMI}=32.3$ ). This tool offered simple, easy to understand, graphic information that significantly reduced nutrition instruction time and increased patient comprehension, evaluated by point-of-care survey (87) response. After IRB approval, a one year follow-up survey was mailed to 70 individuals who were educated on MyPlate. Results of this pilot study demonstrated that the one-time education session lead to an average 4.4 pound weight loss, albeit not statistically significant $p=0.158$. At baseline $26 \%$ followed the MyPlate portion size most or all of the time, compared to $39 \%$ at follow-up. Women were more likely to lose weight than men $\mathrm{p}=0.074$. Participants who saw their MyPlate tool each day trended towards more weight loss than those who did not
\end{abstract}


see their tool daily $\mathrm{p}=0.63$. Barriers to following the MyPlate were cost (48\%) and change in eating habits $(30 \%)$. Health care providers, nursing students, and lay health workers, working with at-risk, vulnerable populations may benefit from using this simple, free, easily accessible, and evidence-based nutrition tool.

Key Words: obesity, nutrition, Appalachia, diabetes 


\section{Introduction}

High rates of disease incidence and mortality are endemic throughout the poor Appalachian regions of the United States. In order to more effectively control the obesity and type 2 diabetes epidemic in this region, more aggressive educational campaigns must be related to the prevention and management of disease. In particular, individuals who are at high-risk of developing type 2 diabetes or who are newly diagnosed are prime candidates for education about lifestyle modification, especially healthy nutrition choices. Studies have shown that people with pre-diabetes who lose weight and increase their physical activity can prevent or delay type 2 diabetes (The DPP group, 2002). This approach has the potential to significantly increase their quality of life. Education must appeal directly to the targeted population and take into account low health literacy and the Appalachian culture and norms.

\section{Background Knowledge}

Obesity rates in the United States (U.S.) have increased dramatically over the last 30 years, and obesity is now an epidemic in the U.S.. Data for 2003-2004 and 2005-2006 indicated that approximately two thirds of U.S. adults and one fifth of U.S. children were either obese (defined for adults as having a body mass index $[\mathrm{BMI}] \geq 30.0$ ) or overweight (defined for adults as BMI of 25.0--29.9 and for children as at or above the $95 \%$ percentile of the sex-specific BMI for age-growth charts) (Ogden, 2006, 2010). Seventytwo percent of men and 64 percent of women are overweight or obese, with about onethird of adults being obese (Flegal, Carroll, Ogden, Curtin, 2010). Poor diet and inactivity are the major contributors to the obesity epidemic (United States Department of Agriculture \& HHS, Dietary Guidelines for Americans, 2010). 
Many chronic diseases, including type 2 diabetes, cardiovascular disease, osteoarthritis and psychological illnesses are related to obesity. Additionally, early death and poor quality of life are associated with obesity (Dixon, 2012). The Trust for America's Health (TFAH) and the Robert Wood Johnson Foundation's $F$ as in Fat Report (2013) estimates if American's diet and exercise habits are not improved, the adult obesity rate in the U.S. will rise to 50-60\% (or higher) in most states by 2030 . In addition to poor health outcomes, obesity accounts for billions of U.S. dollars each year. A study done by Finkelstein, Trogdon, Cohen \& Dietzthe (2009) notes the annual obesity medical burden has risen to almost 10 percent of all medical spending and amounted to $\$ 147$ billion per year in 2008 and the medical costs for people who are obese were $\$ 1,429$ higher than those of normal weight individuals. As a person's BMI increases, so do the number of sick days, medical claims and health care costs associated with that person (Heithoff, Cuffel, Kennedy, Peters, 1997).

\section{Local Problem}

The Appalachian region is a 205,000-square-mile area that follows the Appalachian Mountains from southern New York to northern Mississippi. This region includes 420 counties, and 13 states. Forty-two percent of the region's population is rural, compared with 20 percent of the national population (Appalachian Regional Commission, 2012). Virginians living in the Appalachian region have a higher prevalence of diabetes than those living in non-Appalachian areas. In 2009, diabetes prevalence was $13.9 \%$ among Appalachians, which is significantly higher $(\mathrm{p}<0.001)$ than prevalence of $7.4 \%$ among non-Appalachian Virginians (Virginia Department of Health, 2011). 
A 2010 study estimating the effects of disease prevention on life expectancy in the Appalachia region noted that men would gain 5.7 years and women would gain 4.5 years of life if risk factor exposures (Body Mass Index (BMI), blood glucose, blood pressure and smoking) were at optimal levels (Danaei, Rimm, Oza, Kulkarni, Murray, Ezzati). For BMI improvement alone, adults living in Appalachia would gain 1.5 (male) and 1.4 (female) additional life years. An elevated BMI has an impact on cardiovascular wellness, development of type 2 diabetes and other chronic diseases. A reduction in BMI not only reduces the development of deadly diseases, but also extends life (Danaei, et al, 2010).

High rates of disease incidence and mortality in poor Appalachian regions have been linked to economic factors, but there is also a significant relationship between the cultural and social factors of the population and the health disparities that they face (Brown, et al, 2004). Despite recent advances within the infrastructure of the Appalachian region and continued volunteer efforts directed toward lessening the disparities faced by the population, the region still lags behind the rest of the United States on economic and social indicators (Appalachian Regional Commission, 2012). Long-term poverty and its associated stressors continue to threaten the health of the people in the area. The combination of low socioeconomic status, tobacco use, high obesity rates, limited diet choices, and physical inactivity has contributed to a culture in which poorly controlled chronic diseases are commonplace. Cultural traits tied to Appalachian traditions need to be considered in the development and use of health education materials (Denham, Meyer \& Toborg, 2004). 


\section{Remote Area Medical (RAM)}

For the past 13 years, Remote Area Medical Corps (RAM), a nonprofit, international relief organization based in Knoxville, Tennessee, has sponsored a three day health care event in Wise, Virginia, located in southwest, Virginia, nestled in the Appalachian south central region. A second RAM event, in Grundy, Virginia, also in this region, is a one-and-a-half-day event in October of each year. Both RAM events attract people from a wide area of rural Appalachia, primarily from Virginia, North Carolina, Tennessee and Kentucky. The event's primary organizer, The Health Wagon, is a nonprofit nurse-managed, mobile health care provider in southwest Virginia.

The RAM clinics play a vital role for insured, low wage-earners and for the uninsured by offering services and screening exams they would otherwise forgo due to costly co-payments or lack of coverage by their insurance plans. In July, 2012, the Wise RAM event provided services for 2,442 clients through 5,046 health care encounters that included medical, mammography, eye care, and dentistry. Without these services provided by the 1,379 volunteers, many individuals within this Appalachian region would not receive any health care throughout the year (RAM USA, 2012). A study examining the 2008 Wise RAM population noted the high rates of obesity, heart and vascular disease, diabetes and arthritis that affect this region. During this 2008 event, the average BMI $(n=1,730)$ was 30.9, which is classified as obese. Seventy-seven percent of the clients had a BMI above the normal range: $27 \%$ were classified as overweight and $50 \%$ obese (Snyder \& Milbrath, 2013). 
Although there is a strong correlation between obesity and type 2 diabetes, many Appalachian residents have never received any healthy diet education even though their excess body weight puts them at high-risk for type 2 diabetes (Centers for Disease Control (CDC), 2011; American Diabetes Association, 2012; Dixon, 2012; Trust for America's Health and the Robert Wood Johnson Foundation, 2013). This population's nutrition choices play a role in causing these high levels of obesity because many Appalachians fry much of their food, drink large amounts of sugar-containing soda, and do not regularly practice portion control (Denham, Manoogian \& Schuster, 2007).

\section{Intended Improvement}

The purpose of this project is to evaluate the effectiveness of a one-time educational intervention, the MyPlate Healthy Eating Tool, used at the two 2012 RAM events in southwest Virginia on healthy eating behavior and body weight. At both events, clients at risk of or with type 2 diabetes were instructed using an individually tailored MyPlate educational tool to promote healthy eating. The teaching intervention incorporated concepts from Pender's Health Promotion Model to guide assessment and teaching interventions. The advantage of this education is several fold: 1) it is based on evidence-based findings, 2) the entire educational handouts are readily available on the internet and include on-line data tracking for the users, (www.myplate.gov) 3) all intervention tools are free, 4) the teaching concepts related to MyPlate are straight forward compared to other nutrition instruction methods used for diabetes educationsuch as carbohydrate counting and the exchange diet, and 5) clients who completed a survey immediately after the RAM MyPlate teaching instruction said the tool was easy to comprehend and made healthy eating instruction comprehensible. 


\section{Study Question}

Did vulnerable, obese clients, at risk for type 2 diabetes, living in Appalachia who had a one-time teaching session using the MyPlate Healthy Eating Tool have improved eating behaviors that led to weight loss?

\section{Methods}

A convenience sample was used to test healthy eating knowledge and practice in a group of 87 clients seen at the 2012 RAM mobile clinics in southwest Virginia.

Participants completed a baseline survey offered immediately after the instruction, which addressed current eating practices and perceived success and barriers following the MyPlate tools' recommendations. Follow-up surveys were mailed via U.S. Postal Service one year after instruction to the 70 participants who agreed to be contacted.

\section{Ethical issues}

Client confidentiality was protected by coding the survey responses and separating them from the client record. The mailed follow-up surveys were coded numerically, without any client identifier. The coded responses were correlated to the original survey response which contains only the client medical record number as the client identifier.

The follow-up survey was returned to the researcher in a self-addressed, postage-paid envelope. All returned follow-up surveys were kept in a locked file. The participants were reminded in the cover letter not to put their name on the survey. Only the deidentified survey responses were used by the researcher. Data were coded and entered into an excel database and only aggregate data are reported. There is no conflict of interest with any of the study researchers. 


\section{Setting}

The 2012 Wise RAM event had five nurse educators, all registered nurses, who were educated in client assessment and well trained in tailoring educational interventions to meet the needs of the individual clients. Each educator was assigned to a medical team and was responsible for evaluating each client seen by the team. Both undergraduate and graduate nursing students worked in partnership with the nurse educators to assess and educate the clients seen in the medical clinic. Because of the high prevalence of diabetes, diabetes education was a significant part of the treatment provided by the nurses and students. The nursing students were educated in client assessment, motivational interviewing and teaching the MyPlate method and assessing client comprehension after the education.

\section{Population}

During the 2012 Wise RAM event, a total of 2,442 clients were seen for 5,046 client encounters (RAM USA, 2012). During each RAM event, all attendees are assessed for baseline vital signs, height, weight and random blood sugar measurements. If their blood sugar is in the diabetes range, then an A1C is performed. Common health concerns identified for the adult medical clients seen at the Appalachian RAM events include: diabetes, hypertension, arthritis, poor dentition, depression, heart disease, obesity and pulmonary diseases. The most recent available RAM data which tracked diabetes exclusively are the 2008 Wise clinic, of which $66 \%$ of the 1,038 clients had diabetes or a family history of diabetes, $50 \%$ were obese and $24 \%$ were overweight. This elevated BMI in $74 \%$ of the attendees is above the national average of 66\% (Ogden, 2006, 2010; Snyder \& Milbrath, 2013). 
During the October, 2012 Grundy RAM event, UVA Health System clinicians treated 143 clients (University of Virginia Health Systems, 2012). In Grundy, there was one nurse educator who partnered with two graduate nursing students to educate the clients seen in the medical clinic for the event. Table 1 summarizes the clients seen at both 2012 RAM events (Appendix A).

\section{Planning the intervention}

Client assessment is the key to guide the teaching intervention. Each nurse/client encounter included a client assessment. Pender's Health Promotion Model and motivational interviewing were used to guide the open-ended discussion and to ascertain the nutritional habits (guiding principles) of the client. The educational needs varied for this Appalachian population, but due to the high rates of obesity and overweight people at the RAM clinics, most client education encounters focused on healthy eating. MyPlate tools (Appendix B) were chosen due to their ease of use, evidence-based practice findings which were utilized in tool development, graphically pleasing for all learners, and positive healthy eating messages that correspond to Pender's Health Promotion Model (HPM). This model focuses on individualized client assessments and targeted interventions based on the learning needs of each individual.

Each of the nurse educators used the MyPlate educational tools exclusively for diet and nutrition education. These handouts were both easy to comprehend and allowed the nurse educators to individualize them for client use. They were simple and gave a new meaning to the phrase, "a picture is worth a thousand words." In addition to the handouts a MyPlate 2 foot by 3 foot poster was displayed above the education table for easy reference and viewing. The colorful graphic easily showed the learner that $50 \%$ of 
their plate should contain vegetables and fruits. The instruction time varied from client to client based on many variables including age, educational background, number and extent of health problems, and food preparation abilities. On average, the MyPlate instruction lasted approximately fifteen minutes per client encounter.

The handouts also recommended serving sizes as well as seasonings that may be used as an alternative to sodium (U.S. Department of Agriculture, 2011). The nurses and students explained the MyPlate method and then asked clients if they enjoyed any of the recommended foods and whether they had access to these types of foods. Many of the clients have large gardens where they grow the suggested foods, so use of these foods was strongly reinforced. The nurses and students also worked with the clients to find small ways they could improve their food choices, such as baking rather than frying, and reducing the amount of sugar containing beverages they consume.

The pre-printed MyPlate handouts were $8 \frac{1}{2} 2 \times 11$ inches with the graphic occupying the top $50 \%$ of the page. The bottom $50 \%$ was used for independent goal setting based on client input and nursing assessment. To further educate with the MyPlate, 8 inch paper plates were divided into the four MyPlate colors with a colored magic marker that corresponded to the color-coded MyPlate design. Client food preferences for each of the four plate food groups were written on the plate. Clients were instructed to take the MyPlate graphic home and place it on the refrigerator for two reasons: 1) to generate family support and 2) as a daily reminder of healthy eating choices and portion size. The education emphasis was on making small changes over time to create lifestyle improvement that could lead to improved body weight and/or 
diabetes control, with a long term goal of a $7 \%$ weight reduction to decrease the likelihood of developing type 2 diabetes in those at risk.

At the end of each education session, in order to gauge clients understanding of the information presented, the nurse or student asked the client to make nutritional goals for themselves.

\section{Planning the study of the intervention}

During the 2012 Wise RAM event, sixty-eight adult clients, who were seen in the medical clinic, were educated on the MyPlate method, either by a nurse or student nurse, and completed a written survey. During the 2012 Grundy RAM event, 19 clients who were educated on the MyPlate method completed the baseline, 15 question MyPlate survey. All subjects were over 18 years of age, mentally competent and included both males (28) and females (59). This small sample size serves as a pilot study to determine if the MyPlate educational tool is an effective healthy eating instrument that elicits a behavior change in this population.

The follow-up survey tool was developed based on the initial survey completed by the clients at the time of the MyPlate instruction. Many of the questions are duplicated from initial to follow-up survey and were chosen to determine if this type of instruction is perceived as beneficial to the client and if the client has changed eating habits as a result of the My Plate teaching.

A quasi-experimental (also known as the pre-post intervention) design was used. The study design used a convenience sample to survey clients who were educated at two RAM mobile clinics on the MyPlate method immediately following the instruction. Mail survey follow-up was optional. Institutional Review Board (IRB) approval was obtained 
and a follow-up survey was sent one year after the MyPlate instruction intervention, \#17004. The follow-up survey was sent back to the researcher in a self-addressed, postage-paid envelope to one address at the University of Virginia, School of Nursing.

The primary advantage of this study is that it tested an intervention with a vulnerable population who is at high-risk for developing complications and early death related to their body weight, composition and obesity. This convenience sample illuminates the eating habits and behavior change in this population and can help health care providers and client educators in the region by offering interventions targeted to the population.

\section{Methods of Evaluation}

This convenience sample used a survey with a pre and post design (quasiexperimental study) without a control group. Data collection occurred at baseline, at the time of the intervention to assess participants' eating habits and ability to make healthy nutrition change. The same data were collected one year post intervention. This study only looked at one group of individuals who received the intervention. This study design allows educators to make inferences on the effect of the teaching intervention by analyzing the pre and post survey results.

The results of the nine question follow-up paper and pen survey answers were analyzed and compared to the initial survey responses. Behavior change was compared from baseline to follow-up results by asking respondents about their ability to follow the MyPlate portion size, ability to follow MyPlate by meals: breakfast, lunch and dinner/supper, and the MyPlate tool location to determine if this had an impact on the client's ability to follow the healthy eating recommendations. Barriers to following the 
MyPlate were also assessed at follow-up by using a checkbox list of barriers. Height, weight and calculated BMI were compared from baseline to follow-up. While the follow-up weights are "stated," this has been found to be a reliable indicator of true weight (Shapiro \& Anderson, 2003). Additional healthy eating instruction and individual diets were assessed in the follow-up survey.

\section{Analysis}

The results of the follow-up paper and pen survey were analyzed and compared to the initial survey responses. Behavior change was compared from baseline survey results to follow-up results. Variables included: food portion size, ability to follow MyPlate recommendations by meal, MyPlate educational tool placement, gender, age, body weight and calculated BMI were assessed at follow-up.

All data, including baseline and follow-up survey responses were entered into the excel computer program and the appropriate statistical tests were performed in SPSS (Statistical Product and Service Solutions), version 21 computerized statistical program. Descriptive statistics were calculated for all variables. Height, weight and calculated BMIs were compared for each participant at baseline and follow-up with mean and standard deviation calculated. The "Yes / No" and "True/False" questions are nominal variables and were analyzed using a non-parametric test for the dependent variable group. Responses were coded as ordinal dependent variables " 0 ," " 1 ," and " 2 ," respectively.

\section{Results}

Of the 87 initial survey participants, a maximum of seventy clients responded that they were willing to be contacted for a follow-up survey. After two mailings, 34 surveys were completed and returned to the researchers. Six surveys were returned to sender, 
without a forwarding address. This equals a 53\% survey response rate. Baseline survey results demonstrated that $99 \%$ of the responders said that they found the MyPlate tool easy to comprehend. Eighty-four $(\mathrm{N}=69)$ percent said their family would be able to follow the tool. Ninety-six percent $(\mathrm{N}=83)$ said they could follow this tool when eating at home, while only $64 \%(\mathrm{~N}=55)$ responded they could follow this when eating out. Compared to other diet methods, the MyPlate tool was easier to comprehend, 74\% $(\mathrm{N}=61)$ versus $5 \%(\mathrm{~N}=4), 22 \%(\mathrm{~N}=18)$ had no opinion (Appendix C, figure 1).

Participants were asked at baseline if they would be able to follow the MyPlate. Using a Likert scale, $60 \%(\mathrm{~N}=51)$ said they would be able to follow the MyPlate recommendations. No participants said they could not follow the recommendations.

The baseline and follow-up demographic data are represented in Table 3 (Appendix D). The average age of the 84 participants who answered this question on the baseline survey was 46 and ranged from 19 to 78 years. Sixty-seven percent (67\%), $\mathrm{N}=58$ of the 87 participants at baseline were females and $73 \%(\mathrm{~N}=24)$ at follow-up. The mean BMI at baseline was 32.34 (SD 8.25), with a range of 17-56. The follow-up BMI mean was 31.41 (SD 7.61) with a range of 22-56.8. Further BMI breakdown shows that $21 \%(\mathrm{~N}=18)$ had a BMI $<25$ (considered normal) at baseline, compared with $26 \%(\mathrm{~N}=8)$ at follow-up. At baseline, 23\% ( $\mathrm{N}=19)$ were classified as overweight (BMI >25-29.99) and 19\% (N=6) at follow-up. At baseline, 56\% ( $\mathrm{N}=47)$ were classified as obese (BMI $>=30)$, similar to the $55 \%(\mathrm{~N}=17)$ of respondents at follow-up.

The ability of each client to follow the MyPlate portion size was assessed at baseline and follow-up. At baseline, 26\% ( $\mathrm{N}=22)$ said they follow the MyPlate portion size most or all of the time, while at follow-up, 39\% (N=12) said they follow portion size 
most or all of the time. Even though a higher percentage of responders said they followed MyPlate portion size at follow-up, a higher percentage $(42 \%, \mathrm{~N}=13)$ said they follow MyPlate portion size some of the time or never, compared to $16 \%(\mathrm{~N}=14)$ at baseline (Figure 1, Appendix E).

Based on the follow-up results, respondents identified several barriers to following the MyPlate including cost and a big change in eating habits. Among the follow-up respondents, $46 \%(\mathrm{~N}=15)$ said that cost was a barrier to following MyPlate, compared to $19 \%(\mathrm{~N}=17)$ who perceived this as a barrier at baseline. A McNemar test was used to test significance between both groups and was not found to be significant, probability $=.250$. Another barrier to following MyPlate was, "a big change in eating habits." At baseline, 10\% $(\mathrm{N}=9)$ identified this as a barrier, whereas, 30\% $(\mathrm{N}=10)$ identified it at follow-up, McNemar $=1.0$. A perceived barrier at baseline was a dislike of MyPlate food choices, but this was not reported at follow-up. At baseline, $11 \%(\mathrm{~N}=10)$ said they disliked the recommended food and this was not reported as a barrier by any responders at follow-up. The cost of MyPlate healthy food options and change in eating habits were barriers that were stated more at follow-up than at baseline.

The ability to follow MyPlate by meal was also assessed. Participants were asked at baseline "I can follow MyPlate for breakfast," "I can follow MyPlate for lunch," and "I can follow MyPlate for dinner/supper," with yes/no responses. At follow-up, the questions were, "I follow MyPlate for breakfast," "I follow MyPlate for lunch," and "I follow MyPlate for dinner." Initially participants perceived that they would follow MyPlate more than they actually did. At the time of the MyPlate instruction, participants thought that following MyPlate for all meals would be manageable: $85 \%(n=74)$ for 
breakfast, $89 \%(\mathrm{~N}=74)$ for lunch and 98\% (N=78) for dinner. For follow-up, 59\% $(\mathrm{N}=16)$ of respondents noted that MyPlate was difficult to follow for breakfast, compared to $48 \%(\mathrm{~N}=14)$ for lunch and $43 \%(\mathrm{~N}=12)$ for dinner/super (Figure 2, Appendix F). The most challenging meal to follow was breakfast and that could be related to the MyPlate graphic which includes traditional lunch and dinner pictures versus breakfast items.

Perceived weight loss was assessed by asking the participants if they lost weight from the time of the 2012 RAM event. Eighty-one percent of the participants said they lost weight. Stated weights were used in follow-up and these were compared to baseline weights for all responders. The stated weight was subtracted from the baseline weight to determine weight loss. Based on the recorded weight change, $57 \%$ of the responders lost weight. The mean weight of the responders at baseline was 195.03 pounds $(\mathrm{SD}=42.47)$. The mean weight at follow-up was 190.67 pounds $(\mathrm{SD}=45.62)$. The difference (4.36 pounds weight loss) is not statistically significant, $\mathrm{t}=1.45, \mathrm{df}=31, \mathrm{p}=0.158$ (Figure 3 , Appendix G). The lack of significance could be due to the small sample size. The weight change ranged from -58 to +34 pounds. It was further classified into three categories: weight loss of five or more pounds $(\mathrm{n}=17,53.1 \%)$, stable (within five pounds) $(\mathrm{n}=6$, $18.8 \%)$ and weight gain (gained five or more pounds) $(\mathrm{n}=9,28.1 \%)$.

The relationship between gender and weight loss was explored using Fisher's exact text (due to the limited number of respondents). Among males, four (44.4\%) lost weight, while five (55.6\%) gained weight from baseline to follow-up. Among females, $13(56.5 \%)$ lost five or more pounds from baseline to follow-up, six (26.1\%) remained stable, and four (17.4\%) gained five or more pounds. It appeared that females were more likely to lose weight in comparison with males, Fisher's exact $=5.24, p=0.074$. However, 
due to the small sample size, the result is not statistically significant. The details are summarized in Table 3 (Appendix $\mathrm{H}$ ).

The location of the MyPlate tool was assessed at the one year follow-up. People who kept the MyPlate tool visible reported more weight loss; however, this difference is not statistically significant. Seventeen clients had their MyPlate visible and the average weight loss was 6 pounds (SD14). Fourteen clients did not see their MyPlate tool daily and their mean weight loss was 2.9 pounds (SD21.36) $\mathrm{t}=0.49, \mathrm{df}=29, \mathrm{p}=0.63$. This is detailed in Figure 4, Appendix I. In addition to weight loss and MyPlate visibility, the relationship between BMI change and MyPlate visibility was also assessed. The mean BMI change for the participants who did not have the MyPlate visible was -0.49 (SD3.87). The mean BMI change for those who had the MyPlate visible was -0.876 (SD2.038) $\mathrm{t}=0.49, \mathrm{df}=29, \mathrm{p}=0.63$.

\section{Discussion}

\section{Summary}

Nurses need simple healthy eating solutions when working with obese clients at risk for developing obesity related diseases. This nurse-led pilot study used the MyPlate education tool - a simple, evidence-based, easily accessible, and free tool in a vulnerable, obese, Appalachian population, who are at high-risk or who have type 2 diabetes. The baseline, point-of-care survey results demonstrated that most responders found the MyPlate tool easy to comprehend. The ease of comprehension and reduced teaching time can encourage nurses who have previously not included healthy eating instruction in their practice to consider including it when educating clients. The results of the MyPlate baseline survey demonstrated that the tool was seen as easy to understand and that the 
clients thought they could follow these food recommendations at home and when eating outside the home. There are many immediate clinical implications nurses can incorporate into their plan of care.

This pilot study demonstrated several documented trends, albeit not statistically significant. Weight loss did occur in this population, who received no additional weight loss instruction since the initial MyPlate teaching. An analysis by Sheehan, et al, notes that the mean yearly weight gain for American women and men aged $36-47$ is $0.23 \mathrm{~kg}$ per year, which equals 0.5 pound (2003). In this pilot study, comparisons were made for responders at baseline and follow-up. The lack of significance could be due to the small sample size. This 4.4 pound weight loss is trending in a healthy direction, which is impressive, considering that the evidence suggests that, given this population's age and geographical location, they would be expected to have experienced a weight gain of 0.5 pounds.

Compared to other diet methods, the majority of the participants in this study stated that the MyPlate tool was easier to comprehend compared to other diet methods. In line with Nau (2012), less complex diabetes treatment regimens are associated with higher rates of adherence to diabetes treatment programs. Serono et al (2007) suggested that educational tools geared towards those with diabetes or those at risk of developing diabetes and who reside in medically underserved areas, need to be simple, culturally appropriate, focusing on improving dietary and physical activity behaviors.

Women who were taught the MyPlate method lost more weight than men, although not statistically significant. Gender difference in healthy eating has been documented in the literature. For example, Denham, et al (2004) studied focus groups to 
determine how Appalachian women engage family members in health-promotion activities. The research demonstrated that family was one of the most important themes and that women take an active role in maintaining health among the individual family members. Whittemore (2004) reports that women who participated in diet education interventions demonstrated significantly better diet self-management $(P=0.02)$ compared to men. The weight change between men and women in this MyPlate study may be related to female family member's familiarity with food preparation, as many women in this Appalachian region are the primary food preparers for the household. A study by Wansink \& Kranz noted that women, particularly mothers, are early adapters to the MyPlate (2013). Further MyPlate teaching strategies in this population could include targeted messages related specifically to women as food preparers and food purchasers. By tailoring the education interventions towards women, healthier food choices may impact all family members.

The location of the MyPlate tool in the home had an impact on weight loss; if the tool was seen daily, the weight trended towards a greater loss. A study by Levetan, et al (2002) demonstrated that a visual educational tool placed in a location that was seen daily improved diabetes control. The visible tool also incorporates the Health Promotion Model, which fosters a reinforcement of positive behavior change by keeping "health benefits in front of the client as a reminder that the behaviors in the plan are personally worthwhile and directed toward important life goals" (Pender, et al, 2011, p. 129).

Further analysis was done on MyPlate visibility and weight change by categories: lost five or more pounds, stable within five pounds, or gained five or more pounds. Most of the participants who had their MyPlate tool visible on a daily basis lost five or more 
pounds. Although this is not statistically significant, the Cramers' V indicates a moderate effect size, which has implications for nursing practice. Further studies are needed to determine if the MyPlate tool location has an impact on weight loss and behavior change. Making the educational tools visible is easy, simple and free. This effect size could have important clinical implications for this region and population and other vulnerable populations.

At baseline, participants believed that MyPlate could be followed for all meals. One year after instruction, the ability to follow MyPlate by meal was less than what was anticipated by the participants. Breakfast is the most challenging meal to follow the recommendations for proper nutrient distribution. Future teaching interventions should incorporate strategies that include breakfast balanced plates; for example, graphics should be used to show a healthy balanced breakfast plate, and breakfast food models. Specific breakfast items should be assessed by the nurse. Clients should be provided with written examples of MyPlate breakfast foods. Use of these visual reminders is supported by the HPM recommendation to develop a behavior-change plan.

The post survey results, participants reported two barriers to following the MyPlate: cost and a big change in eating habits. The HPM suggests that perceived barriers are important determinants of health-promoting behavior. The responses to the follow-up survey are congruent with the HPM's perceived barriers. Understanding these barriers can help the nurse tailor participant education and encourage dialogue to address perceived barriers to healthy eating, with a focus on solutions. Examples include: low cost fruits, vegetables, whole grains, low fat protein sources and gradual incorporation of healthy foods into the diet to encourage long-term behavior change. 
Using the HPM to guide the MyPlate intervention encouraged educators to tailor the MyPlate materials to the individual, by addressing favorite foods, individual eating habits, food preparation and family involvement. Future interventions can address these potential barriers during the nursing assessment and guide the discussion towards overcoming these barriers to healthy eating. Other vulnerable populations may experience similar barriers to following MyPlate. Nurses can incorporate specific examples of cost savings, including inexpensive food options, substitutes for higher cost foods can be taught during client education sessions.

Other vulnerable populations may experience similar barriers to following MyPlate. Nurses can incorporate specific examples of cost savings, including inexpensive food options, substitutes for higher cost foods, like fruit can be taught during client education sessions.

The perceived ability to follow MyPlate by meal and the actual ability to follow by meal was assessed and found that MyPlate was more challenging to follow than anticipated. Likewise, at follow-up $81 \%$ of the responders said they had lost weight however actual weight loss showed that $57 \%$ of the responders actually lost weight.

Participants were more optimistic about their perceived ability to follow the treatment plan than their actual ability to follow it. And, participants thought they lost more weight than they actually did. In future education sessions about My Plate, nurses should assess a client's ability to follow the MyPlate, but understand that a client's belief and desire to change behavior may be more optimistic than reality. Nurses can target the teaching interventions with these perceived beliefs in mind, as the client may become discouraged if their progress towards their healthy eating goal is not met. Incorporating 
concepts of the HPM into targeted interventions for a behavior change plan, will allow the nurse and client to work towards realistic and achievable goals.

\section{Study Limitations}

One limitation is the convenience sample, which may show an under or over representation of the population. A second limitation related to the "stated weight" versus an actual weight in the follow-up survey. According to Shapiro \& Anderson (2003), selfreported weights are an accurate measure of true weight. A third limitation is the written survey; the respondent may not understand a question in the survey. This was addressed by re-wording the follow-up survey from the baseline survey, for example, the Likert scale was confusing for some respondents, so this was removed from the follow-up survey. A third limitation is the lack of race data collected at baseline. Based on the U.S. Census data (2012), each of the Virginia counties represented at the RAM clinics consists of a Caucasian population great than $93 \%$. A fourth limitation is the length of time between MyPlate education and survey follow-up. Finally, the small sample size could have impacted significance.

\section{Conclusions}

The results of this pilot study have implications for an immediate and impressive impact on nursing practice. As health educators, nurses are often the first health care professionals that deal with clients on an intimate level. The public has a great deal of trust in nurses. The effectiveness of the MyPlate tool is far reaching, as there are limited health care providers in this Appalachian region. The evidence demonstrates that both obesity and type 2 diabetes in the Appalachian region leads to shorter life expectancy and chronic, painful and expensive diseases. Nurses are well equipped with knowledge, 
assessment and communication skills to educate clients and communities on healthy eating.

In order to more effectively control the obesity and type 2 diabetes epidemic in Appalachia, more aggressive educational campaigns must be initiated in the region related to the prevention and management of the disease. In particular, individuals who are at high-risk of developing type 2 diabetes or are newly diagnosed are prime candidates for education about lifestyle modification, especially healthy nutrition choices. Studies have shown that people with pre-diabetes who lose weight and increase their physical activity can prevent or delay type 2 diabetes (The DPP group, 2002). This approach has the potential to significantly increase their quality of life. Education must appeal directly to the targeted population and take into account low health literacy and the Appalachian culture and norms.

The MyPlate intervention that was evaluated here used the Health Promotion Model to guide assessment and interventions. Using the Health Promotion Model to develop targeted nursing assessment and interventions that focus on positive behavior change by empowering clients to make healthy, realistic and achievable goals, can help nurses in a variety of health care settings develop a health promotion plan for clients or the community. These steps are easily adaptable in many clinical settings; including hospitals, clinics, parish nursing, public health departments, community health centers, free clinics and schools. Nurses can and should develop interventions that address preventative measures related to the chronic disease and obesity epidemic. Future studies are needed to replicate this study in larger samples and in a variety of health care settings. 
There were several results that can be immediately incorporated into client education to effectively improve healthy eating. First, all clients should be encouraged to place their educational materials in a location that will be viewed each day. Second, the role of the female as food preparer and family caregiver should be addressed when teaching Appalachian clients, particularly females. Specific suggestions for MyPlate food choices at all meals should be addressed, particularly breakfast. Portion size, perceived and actual, should be discussed to guide clients in meeting their healthy eating goals. And lastly, barriers to healthy food choices should be addressed in all MyPlate teaching sessions, as these barriers have an impact on client success.

\section{Funding}

A grant from The University of Virginia Alumni Association was used for participant incentives. 


\section{References}

American Diabetes Association (2012). Position Statement: Standards of Medical Care in Diabetes-2012 Diabetes Care, 35, S11-S63; doi:10.2337/dc12-s011

Appalachian Regional Commission (2006-2011) (2012). Retrieved from: www.arc.gov/ Battaglia,M.P., Khare, M., Frankel, M.R., Murray,M.C., Buckley, P. \& Peritz,S.(2008). Response rates: How have they changed and where are they headed? In Wiley, J.(Ed.), Advances in telephone survey methodology.

DOI: 10.1002/9780470173404.ch24. Retrieved from:

http://onlinelibrary.wiley.com.proxy.its.virginia.edu/doi/10.1002/9780470173404. $\operatorname{ch} 24 /$ summary

Brown, AF, Ettner, SL, Piette, J, Weiberger, M, Gregg, E, Shapiro, MF, Karter, AJ, Safford, M., Waitzfelder, B, Prata, PA, Beckles, GL. (2004). Socioeconomic position and health among persons with type 2 diabetes: A conceptual model and Review of the literature. Epidemiological Reviews, 26, 63-

77.doi:10.1093/epirev/mxh002

Centers for Disease Control and Prevention (2011). National diabetes fact sheet: national estimates and general information on diabetes and prediabetes in the United States. Atlanta, GA: U.S. Department of Health and Human Services, Centers for Disease Control and Prevention.

Danaei G, Rimm EB, Oza S, Kulkarni SC, Murray CJL, et al. (2010). The Promise of Prevention: The Effects of Four Preventable Risk Factors on National Life Expectancy and Life Expectancy Disparities by Race and County in the United States. PLoS Med 7(3): e1000248. doi:10.1371/journal.pmed.1000248 
Denham, S. A., Manoogian, M. M., \& Schuster, L. (2007). Managing family support and dietary routines: Type 2 diabetes in rural appalachian families. Families, Systems, \& Health, 25(1), 36-52. doi: 10.1037/1091-7527.25.1.36

Denham, S., Meyer, M. \& Toborg, M. (2004). Tobacco cessation in adolescent females in Appalachian communities. Family \& Community Health, 27(2), 170-181.

Denham, S. A., Meyer, M. G., Toborg, M. A., \& Mande, M. J. (2004). Providing health education to appalachia populations. Holistic Nursing Practice, 18(6), 293-301.

"Diabetes in Appalachia" (2011). Retrieved from: http://www.diabetesfamily.net/

Dixon, J.B. (2010). The effect of obesity on health outcomes. Molecular Cell Endocrinology, 316(2),104-8.

DPP Program Group. (2002). Reduction in the incidence of type 2 diabetes with lifestyle intervention or metformin. New England Journal of Medicine, 346, 393-403.

Edwards, P., Roberts, I., Clarke, M., DiGuiseppi, C., Pratap, S., Wentz, R., \& Kwan, J. (2002). Increasing response rates to postal questionnaires: systematic review. BMJ, 327, 1183-1192. Retrieved from: http://www.bmj.com/content/324/7347/1183

Finkelstein, E.A., Trogdon,J.G., Cohen, J.W., Dietz, W. (2009). Annual Medical Spending Attributable To Obesity: Payer-And Service-Specific Estimates. Health Affairs, 28(5), 822-831.

Flegal KM, Carroll MD, Ogden CL, Curtin LR. (2010). Prevalence and trends in obesity among U.S. adults, 1999-2008. JAMA,303(3), 235-241.

Heithoff, K.A., Cuffel, B.J., Kennedy, S., Peters, J. (1997). The Association Between Body Mass and Health Care Expenditures. Clinical Therapy, 19(4):811-820. 
Levetan, C. Dawn, K., Robbins, D., Ratner, R. (2002) Impact of Computer-Generated Personalized Goals on HbA1c. Diabetes Care, 25(1), 2-8.

Ogden CL, Carroll, MD, Curtin, LR, et al. (2006). Prevalence of overweight and obesity in the United States, 1999--2004. JAMA, 295, 1549-55.

Ogden, C. L., \& Carroll, M. D. (2010). Prevalence of overweight, obesity, and extreme obesity among adults: United States, trends 1960-1962 through 2007-2008. National Center for Health Statistics, 6, 1-6.

Pender NJ, Murdaugh CL, Parsons MA. (2011). Health Promotion in Nursing Practice (6th ed). Upper Saddle River, NJ: Prentice Hall.

RAM USA (2012). Available from: http://www.ramusa.org/

Shapiro, J., Anderson, D. (2003). The effects of restraint, gender and body mass index on the accuracy of self-reported weight. Int J Eat Disor, 34,177-180.

Sheehan, T.J., DuBrava, S., DeChello, L.M., Fang, Z. (2003). Rates of weight change for black and white Americans over a twenty year period. International Journal of Obesity, 27,498-504.

Snyder, A.E., Milbrath, G.R. (2013). Description of healthcare needs at an episodic clinic in rural southwest Virginia. Rural and Remote Health, 13, 2557. Retrieved from: http://www.rrh.org.au

Trust for Americans Health and the Robert Wood Johnson Foundation (2013). F as in Fat: How obesity Threatens America's future, 2013 report. Retrieved from: http://fasinfat.org/

U.S Census Bureau (2012). http://quickfacts.census.gov/qfd/states/51/51195.html 
U.S. Department of Agriculture and U.S. Department of Health and Human Services (2010). Dietary Guidelines for Americans, 2010. $7^{\text {th }}$ Edition, Washington, DC: U.S. Government Printing Office.

University of Virginia Health Systems (2012). Retrieved from: https://uvahealth.com/about/community/ram/ram-grundy

UVa today (2009). Retrieved from: http://www.news.virginia.edu/content/uva-healthsystem-planning-20-percent-increase-patients-seeking-care-ram-clinic Virginia Department of Health (2011). Diabetes in Virginia. Richmond, VA: VDH, Division of Prevention and Health Promotion. Retrieved from: https://www.vdh.virginia.gov/ofhs/Prevention/diabetes/documents/2012/pdf/Diab etes\%20Burden\%20Report1.pdf

Wansink, B., \& Kranz, S. (2013). Who's using MyPlate? Journal of Nutrition Education and Behavior, 45(6), 728-732. doi:10.1016/j.jneb.2013.03.009

Whittemore R, Melkus GD, Sullivan A, Grey M. (2004). A nurse-coaching intervention for women with type 2 diabetes. Diabetes Educator; 30(5),795-804. 
Appendix A

Table 1. 2012 RAM Participants Seen at Both Medical Clinics.

\begin{tabular}{|l|c|c|}
\hline & Wise RAM - July 2012 & Grundy RAM - October 2012 \\
\hline $\begin{array}{l}\text { Clients seen in the medical } \\
\text { clinic }\end{array}$ & 2,619 & 143 \\
\hline Clients seen by an educator & 297 & 60 \\
\hline $\begin{array}{l}\text { Clients with diabetes seen } \\
\text { by an educator }\end{array}$ & 115 & 36 \\
\hline MyPlate instruction & 107 & 19 \\
\hline $\begin{array}{l}\text { Clients who filled out the } \\
\text { MyPlate survey }\end{array}$ & 68 & 16 \\
\hline $\begin{array}{l}\text { Clients willing to have } \\
\text { follow-up MyPlate survey }\end{array}$ & 57 & \\
\hline
\end{tabular}




\section{Appendix B}

MyPlate, U.S. Department of Agriculture, 2011

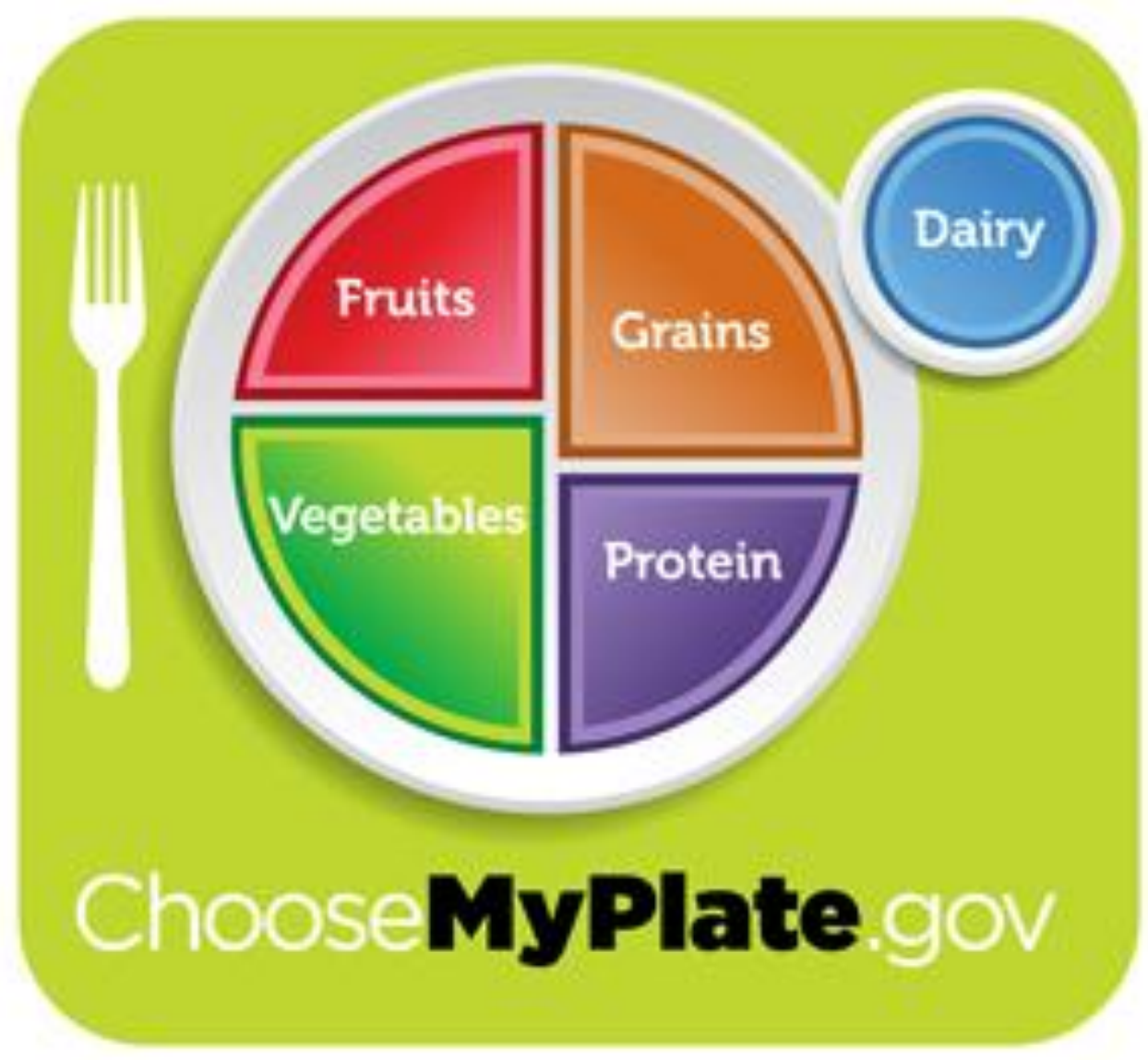

Retrieved from: www.myplate.gov 
Appendix C

Figure 1: MyPlate comprehension

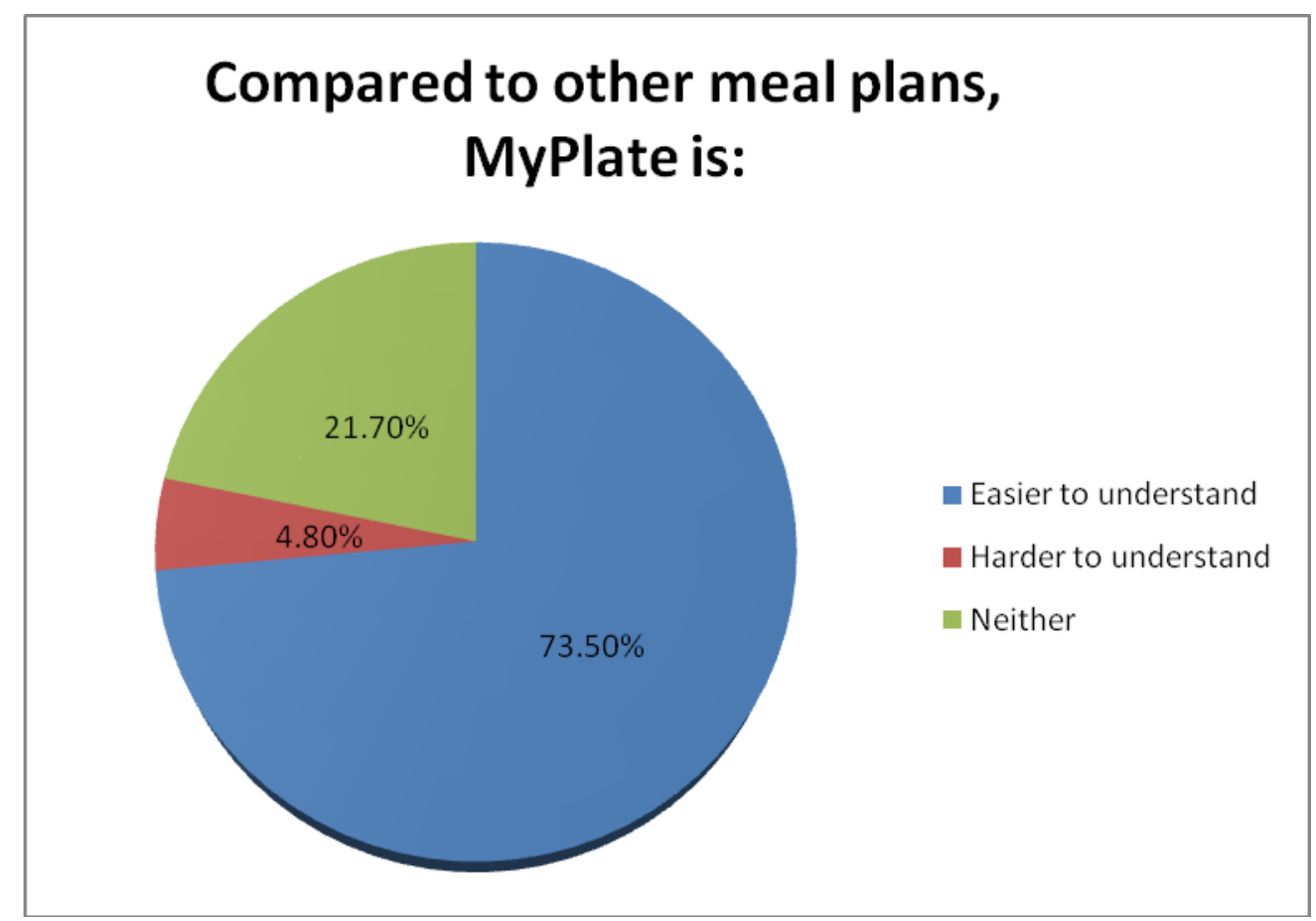

Results based on the survey given to 87 clients instructed on the MyPlate method at the 2012 RAM events. 


\section{Appendix D}

Table 2: Baseline and Follow-up Participant Characteristics

Variables

\begin{tabular}{|c|c|c|c|c|}
\hline & \multicolumn{2}{|r|}{ Baseline } & \multicolumn{2}{|r|}{ Follow-up } \\
\hline & $\begin{array}{c}\text { Total } \\
\text { Number }\end{array}$ & $\begin{array}{c}\text { Number (\%) OR } \\
\text { Mean (SD) }\end{array}$ & $\begin{array}{c}\text { Total } \\
\text { Number }\end{array}$ & $\begin{array}{c}\text { Number (\%) OR } \\
\text { Mean (SD) }\end{array}$ \\
\hline Male & & $29(33 \%)$ & & $9(27 \%)$ \\
\hline Female & & $58(67 \%)$ & & $24(73 \%)$ \\
\hline Age & 84 & $\begin{array}{c}\text { Mean: } 46.05(12.87) \\
\text { Range: } 19-78\end{array}$ & 33 & $\begin{array}{c}\text { Mean: } 46.97(10.06) \\
\text { Range: } 22-63\end{array}$ \\
\hline BMI & 84 & $\begin{array}{l}\text { Mean: } 32.34(8.25) \\
\text { Range: } 17.01-55.78\end{array}$ & $31 *$ & $\begin{array}{c}\text { Mean: } 31.41(7.61) \\
\text { Range: } 21.92-56.83\end{array}$ \\
\hline - $\quad$ Normal $(<25)$ & & $18(21 \%)$ & & $8(26 \%)$ \\
\hline - $\quad$ Overweight (25-29.99) & & $19(23 \%)$ & & $6(19 \%)$ \\
\hline - $\quad$ Obese $(>=30)$ & & $47(56 \%)$ & & $17(55 \%)$ \\
\hline
\end{tabular}

*One participant did not supply height; one did not supply follow-up weight. 
Appendix E

Figure 1: Ability to Follow MyPlate Portion Size at baseline and follow-up

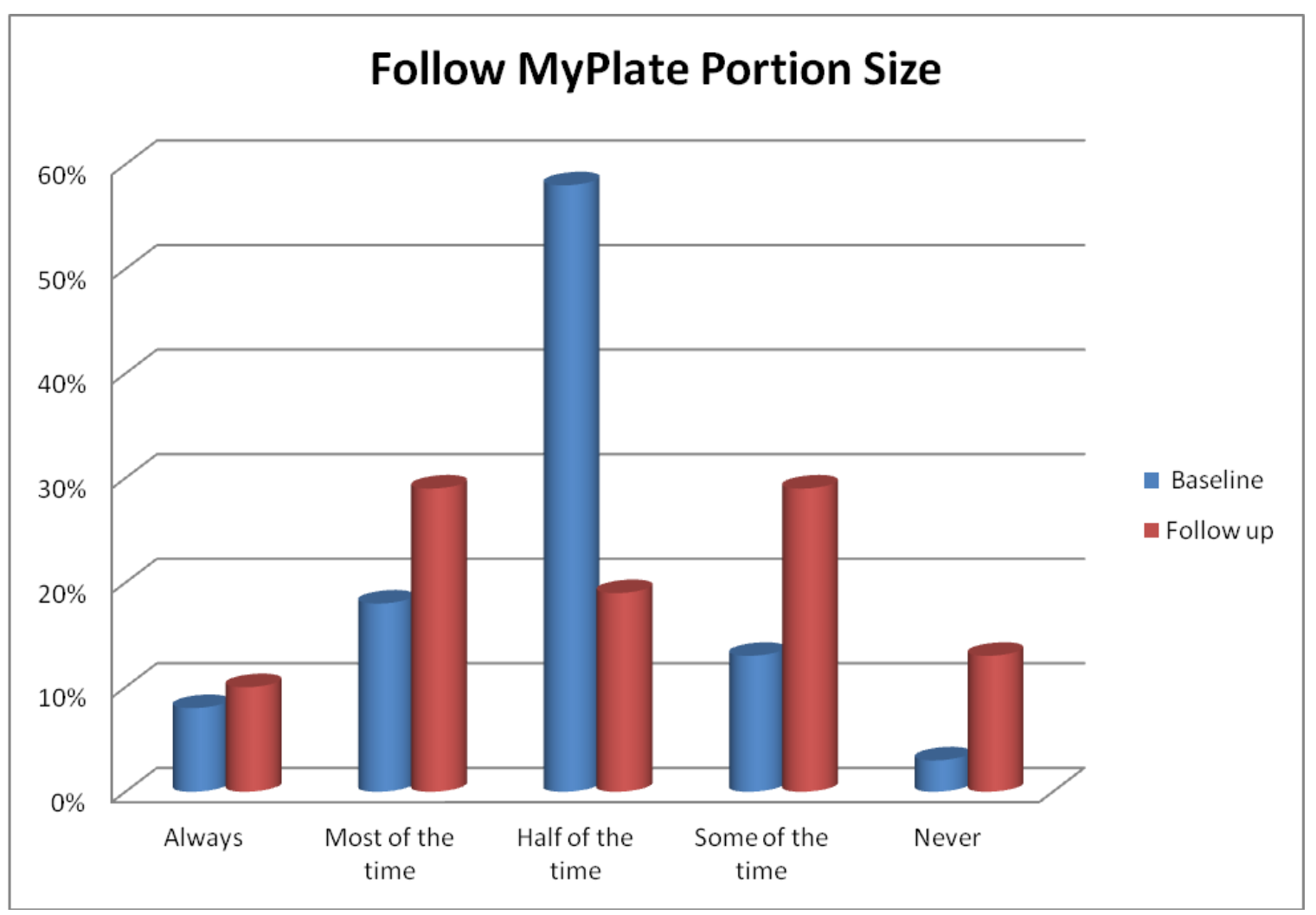

Participants were asked at baseline, "This is how I eat now", with a Likert scale response. At follow-up, the Likert scale was changed to the above label check off. This change in measurement (Likert scale to check list) may account for the large discrepancy. 
Appendix F

Figure 2: Perceived and Actual Ability to Follow MyPlate by Meal

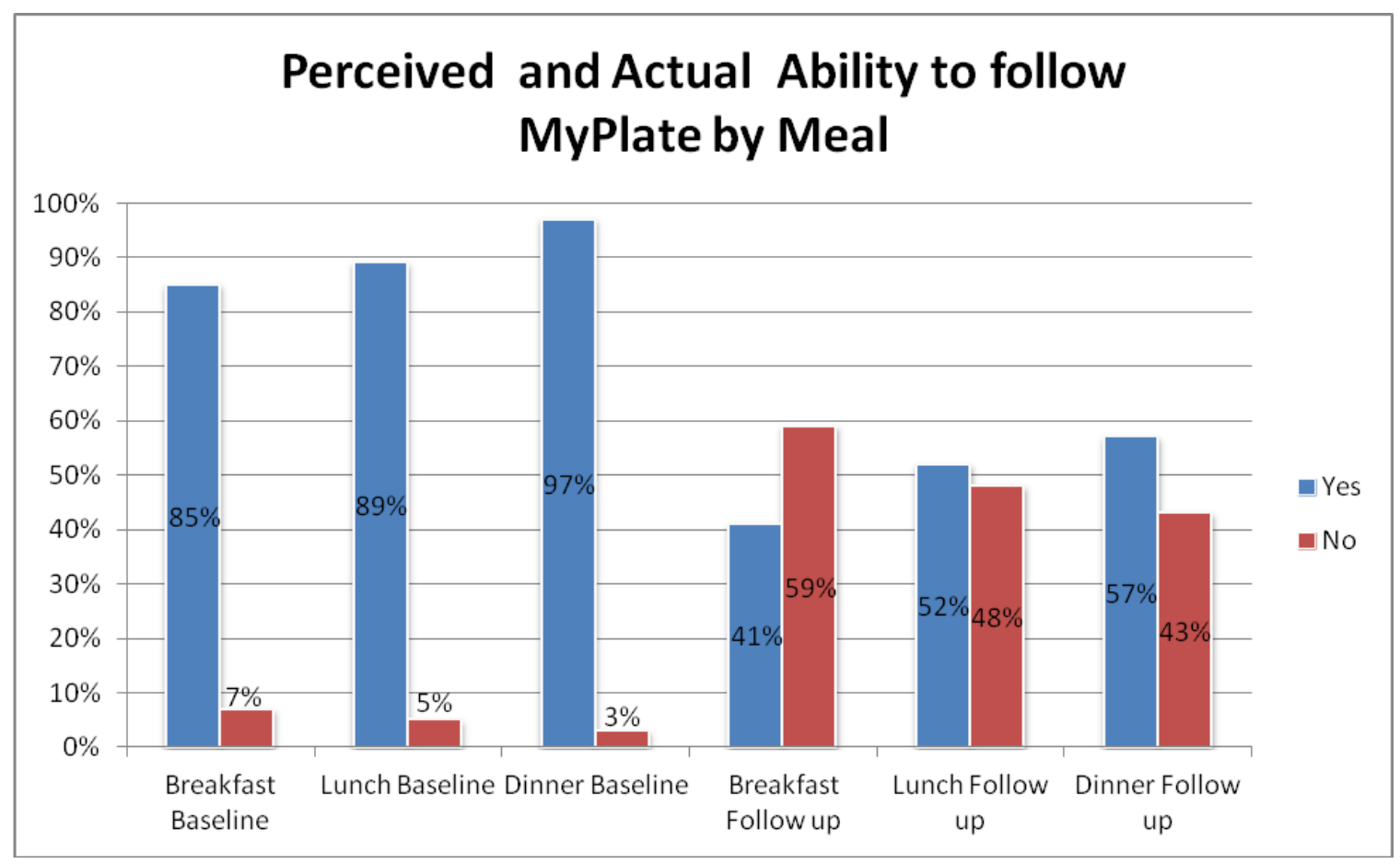

Participants were asked at baseline "I can follow MyPlate for breakfast," "I can follow MyPlate for lunch," and "I can follow MyPlate for dinner/supper." At follow-up, the questions were, "I follow MyPlate for breakfast," "I follow MyPlate for lunch," and "I follow MyPlate for dinner.” Participants perceived they would follow MyPlate more than they actually did. 
Appendix G

Figure 3: Responder Weight Loss in pounds at Baseline and Follow-up

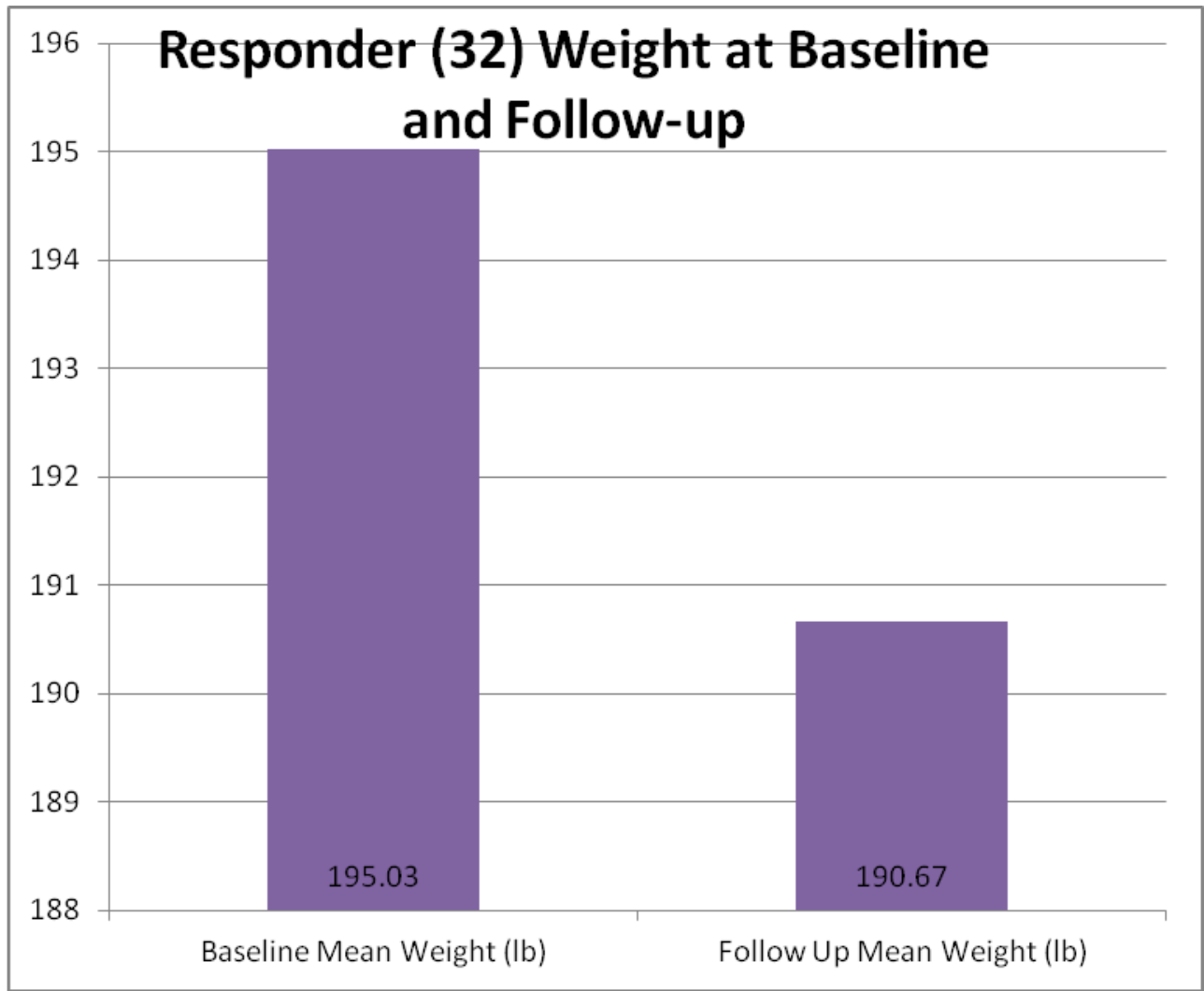

Mean baseline weight was 195.03 pounds $(\mathrm{SD}=42.47)$. The mean weight for follow-up was 190.67 pounds $(\mathrm{SD}=45.62)$. The differnce (4.36 pounds weight loss) is not statistically significant, $\mathrm{t}=1.45, \mathrm{df}=32, \mathrm{p}=0.158$. 
Appendix $\mathrm{H}$

Table 3: Gender Difference and Weight Change

\begin{tabular}{|c|c|c|c|c|}
\hline & $\begin{array}{c}\text { Lost 5 } \\
\text { pounds or } \\
\text { more } \\
\mathrm{N}(\%)\end{array}$ & $\begin{array}{c}\text { Stable Wt, } \\
\text { within 5 } \\
\text { pounds } \\
\text { change } \\
\mathrm{N}(\%)\end{array}$ & $\begin{array}{c}\text { Gained 5 } \\
\text { pounds or } \\
\text { more } \\
\mathrm{N}(\%)\end{array}$ & total \\
\hline Males & $4(44 \%)$ & 0 & $5(56 \%)$ & 9 \\
\hline Females & $13(57 \%)$ & $6(26 \%)$ & $4(17 \%)$ & 23 \\
\hline $\begin{array}{c}\text { Participants in each } \\
\text { weight category }\end{array}$ & $17(53 \%)$ & $6(19 \%)$ & $9(28 \%)$ & 32 \\
\hline
\end{tabular}

Fisher's exact $=5.24, \mathrm{p}=0.074$. 
Appendix I

Figure 4: MyPlate Tool Visibility and Mean Pound Weight Loss

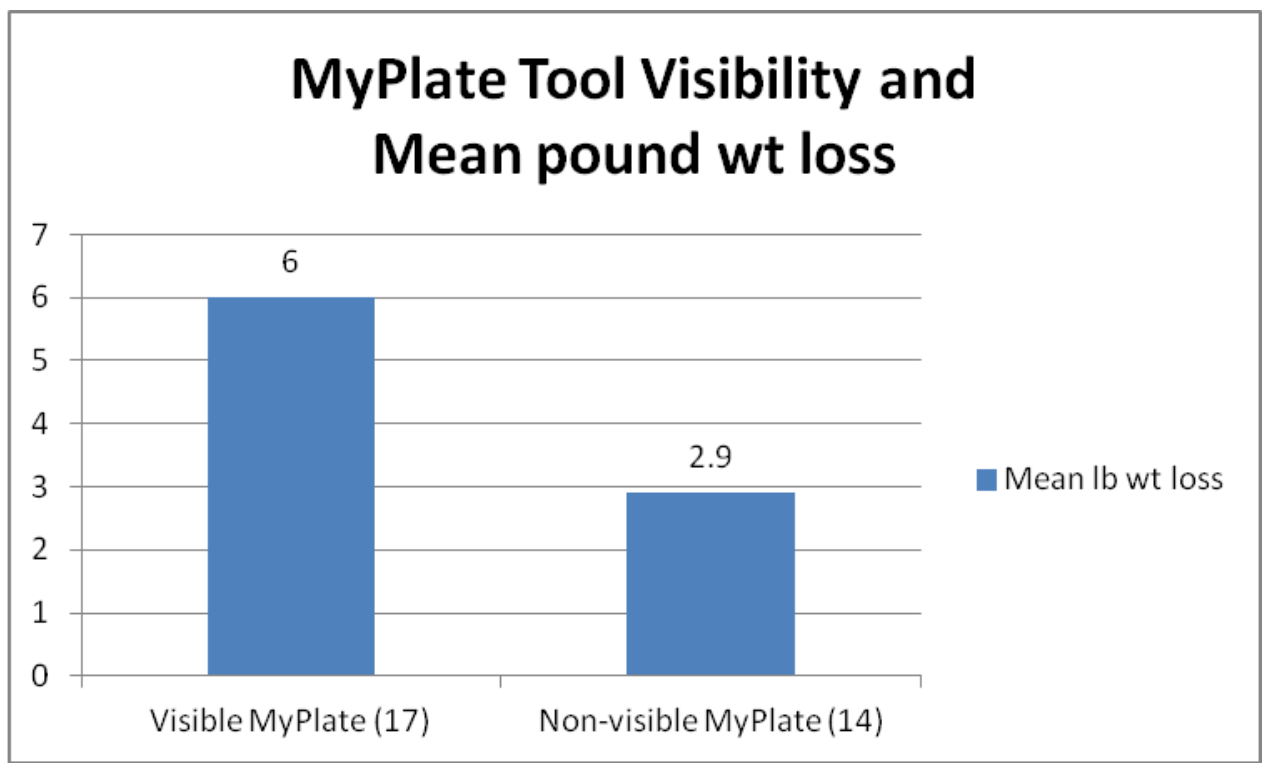

Weight loss in pounds, $\mathrm{t}=0.49, \mathrm{df}=29, \mathrm{p}=0.63$ 